\title{
HAT-TR-318-007: A Double-lined M Dwarf Binary with Total Secondary Eclipses Discovered by HATNet and Observed by $\mathrm{K2}^{* \dagger}$
}

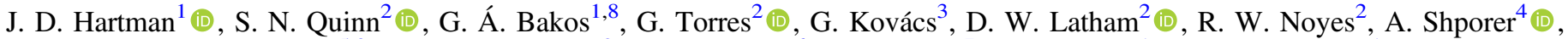

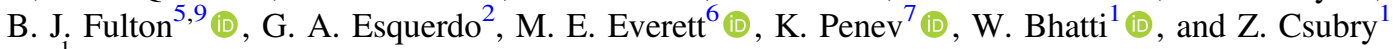 \\ ${ }^{1}$ Princeton University, Department of Astrophysical Sciences, Princeton, NJ, USA; jhartman@astro.princeton.edu \\ ${ }^{2}$ Harvard-Smithsonian Center for Astrophysics, Cambridge, MA 02138, USA \\ ${ }^{3}$ Konkoly Observatory of the Hungarian Academy of Sciences, Budapest, Hungary \\ ${ }^{4}$ Jet Propulsion Laboratory, California Institute of Technology, 4800 Oak Grove Drive, Pasadena, CA 91109, USA \\ ${ }^{5}$ Institute for Astronomy, University of Hawaii, Honolulu, HI 96822, USA \\ ${ }^{6}$ National Optical Astronomy Observatory, Tucson, AZ, USA \\ ${ }^{7}$ Department of Physics, The University of Texas at Dallas, Richardson, TX, USA \\ Received 2017 November 17; revised 2018 January 9; accepted 2018 January 10; published 2018 February 14
}

\begin{abstract}
We report the discovery by the HATNet survey of HAT-TR-318-007, a $P=3.34395390 \pm 0.00000020$ day period detached double-lined $\mathrm{M}$ dwarf binary with total secondary eclipses. We combine radial velocity (RV) measurements from TRES/FLWO $1.5 \mathrm{~m}$ and time-series photometry from HATNet, FLWO $1.2 \mathrm{~m}, \mathrm{BOS} 0.8 \mathrm{~m}$, and NASA $K 2$ Campaign 5, to determine the masses and radii of the component stars: $M_{\mathrm{A}}=0.448 \pm 0.011 \mathcal{M}_{\odot}^{\mathrm{N}}$, $M_{\mathrm{B}}=0.2721_{-0.0042}^{+0.0041} \mathcal{M}_{\odot}^{\mathrm{N}}, R_{\mathrm{A}}=0.4548_{-0.0036}^{+0.0035} \mathcal{R}_{\odot}^{\mathrm{N}}$, and $R_{\mathrm{B}}=0.2913_{-0.0024}^{+0.0023} \mathcal{R}_{\odot}^{\mathrm{N}}$. We obtained a FIRE/Magellan near-infrared spectrum of the primary star during a total secondary eclipse, and we use this to obtain disentangled spectra of both components. We determine spectral types of $\mathrm{ST}_{\mathrm{A}}=\mathrm{M} 3.71 \pm 0.69$ and $\mathrm{ST}_{\mathrm{B}}=\mathrm{M} 5.01 \pm 0.73$ and effective temperatures of $T_{\text {eff,A }}=3190 \pm 110 \mathrm{~K}$ and $T_{\text {eff, }}=3100 \pm 110 \mathrm{~K}$ for the primary and secondary star, respectively. We also measure a metallicity of $[\mathrm{Fe} / \mathrm{H}]=+0.298 \pm 0.080$ for the system. We find that the system has a small, but significant, nonzero eccentricity of $0.0136 \pm 0.0026$. The $K 2$ light curve shows a coherent variation at a period of $3.41315_{-0.00032}^{+0.00030}$ days, which is slightly longer than the orbital period, and which we demonstrate comes from the primary star. We interpret this as the rotation period of the primary. We perform a quantitative comparison between the Dartmouth stellar evolution models and the seven systems, including HATTR-318-007, that contain $\mathrm{M}$ dwarfs with $0.2 \mathcal{M}_{\odot}^{\mathrm{N}}<M<0.5 \mathcal{M}_{\odot}^{\mathrm{N}}$, have metallicity measurements, and have masses and radii determined to better than $5 \%$ precision. Discrepancies between the predicted and observed masses and radii are found for three of the systems.
\end{abstract}

Key words: binaries: eclipsing - stars: fundamental parameters - stars: individual (HAT-TR-318-007, EPIC 211432946) - stars: late-type

Supporting material: machine-readable tables

\section{Introduction}

Detached double-lined eclipsing binary systems are fundamental to our understanding of stellar evolution. By applying simple geometry and orbital mechanics, it is possible to measure the masses and radii of the component stars in such a system. Assuming that both stars are the same age, one can then test a theoretical model by checking whether there is an age at which the model would predict that two stars with the measured masses would have the measured radii. These models also predict the temperatures (or luminosities) of the stars and depend on the composition of the stars. A sharper test of the model can be performed if the temperatures and metallicities (a.k.a. atmospheric parameters) of the component stars can be measured spectroscopically.

\footnotetext{
* Based in part on observations made with the Nordic Optical Telescope, operated on the island of La Palma jointly by Denmark, Finland, Iceland, Norway, and Sweden, in the Spanish Observatorio del Roque de los Muchachos of the Instituto de Astrofisica de Canarias.

$\dagger$ This paper includes data gathered with the $6.5 \mathrm{~m}$ Magellan Telescopes located at Las Campanas Observatory, Chile.

${ }^{8}$ Packard Fellow.

9 NSF Postdoctoral Fellow.
}

The double-lined nature of the spectrum, which enables the measurement of the masses of both components of a binary, complicates the measurement of the stellar atmospheric parameters. Although algorithms exist to disentangle the composite spectra into spectra of the individual component stars (Simon \& Sturm 1994; Hadrava 1995), determining the continuum level of each component is difficult, leading to systematic uncertainties in the depths of the absorption lines and hence in the atmospheric parameters. In rare cases the primary or secondary eclipse may be total, in which case one can obtain an uncontaminated spectrum of one component star during total eclipse, providing an opportunity to cleanly measure the atmospheric parameters of the star. This spectrum also serves as an ideal template for disentangling the out-ofeclipse composite spectra, allowing one to obtain a spectrum for the totally eclipsed star as well. Such a technique was applied by Terrien et al. (2012a) to the late M dwarf eclipsing binary system CM Dra. Although this object does not exhibit total eclipses, the eclipses are close enough to totality that it is possible to obtain an effectively uncontaminated spectrum of a single component during eclipse. Terrien et al. (2012a) measured a subsolar metallicity of $[\mathrm{Fe} / \mathrm{H}]=-0.30 \pm 0.12$ for CM Dra based on these observations. Feiden \& Chaboyer (2014), however, conclude that CM Dra may have a 0.2 dex 
Table 1

Summary of Time-series Photometry for HAT-TR-318-007

\begin{tabular}{|c|c|c|c|c|c|c|}
\hline Facility & Date(s) & Event(s) & \# Obs. & Filter & $\begin{array}{c}\text { Median Cadence } \\
\text { (s) }\end{array}$ & $\begin{array}{c}\text { rms Precision } \\
\text { (mag) }\end{array}$ \\
\hline HAT-5/G317 & 2010 Nov-2011 Apr & Both & 4167 & $r$ & 236 & 0.166 \\
\hline HAT-8/G317 & 2010 Nov-2011 Apr & Both & 4289 & $r$ & 239 & 0.166 \\
\hline HAT-6/G318 & 2008 Dec-2009 May & Both & 2972 & $r$ & 352 & 0.149 \\
\hline HAT-7/G365 & 2010 Nov-2011 May & Both & 8115 & $r$ & 235 & 0.348 \\
\hline HAT-8/G365 & 2011 Apr-2011 May & Both & 452 & $r$ & 232 & 0.348 \\
\hline НAT-6/G366 & 2010 Nov-2011 Apr & Both & 4334 & $r$ & 236 & 0.142 \\
\hline НAT-9/G366 & 2010 Nov-2011 Apr & Both & 6011 & $r$ & 230 & 0.142 \\
\hline FLWO $1.2 \mathrm{~m}$ & 2011 Mar 30 & Secondary & 123 & $i$ & 133 & 0.005 \\
\hline FLWO $1.2 \mathrm{~m}$ & 2011 Dec 29 & Secondary & 327 & $i$ & 59 & 0.006 \\
\hline FLWO $1.2 \mathrm{~m}$ & 2012 Jan 03 & Primary & 464 & $i$ & 44 & 0.008 \\
\hline BOS $0.8 \mathrm{~m}$ & 2012 Jan 03 & Primary & 111 & $i$ & 140 & 0.013 \\
\hline $\mathrm{K} 2 \mathrm{C}^{\mathrm{a}}$ & 2015 Apr-Jul & Both & 3298 & $K p$ & 1765 & 0.005 \\
\hline
\end{tabular}

Note.

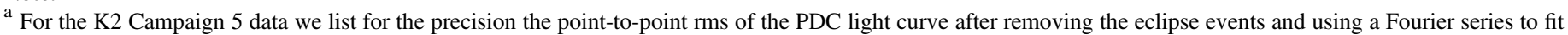

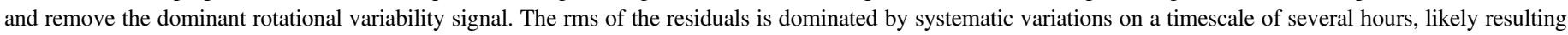
from inaccuracies in removing the instrumental variations due to the spacecraft roll for such a large-amplitude variable star.

enhancement in $\alpha$-elements compared to the Sun, in which case its $[\mathrm{Fe} / \mathrm{H}]$ metallicity is close to solar.

Another advantage of binaries with total eclipses is that the radii of the component stars can often be measured with greater precision than can be done for grazing systems owing to the additional information provided by the times of second and third contact (end of ingress and start of egress, respectively). The contact points can be measured quite precisely owing to the sharp change in light-curve morphology at these times. Moreover, the determination of the times of contact is relatively insensitive to the presence of spots on one or both of the stars, which may be a significant source of systematic errors in determining the radii of $\mathrm{M}$ dwarf stars in grazing eclipsing binaries (Morales et al. 2010; Windmiller et al. 2010).

Over the past decade there has been a significant observational effort to determine the fundamental parameters of $\mathrm{M}$ dwarf stars, which, due to their faintness, have not been well studied until recently. The first precise determinations of the fundamental parameters for early and mid-M dwarfs in the eclipsing systems YY Gem and CU Cnc indicated that the components of these systems have radii that are $10 \%-20 \%$ larger than predicted by theoretical models (Torres \& Ribas 2002; Ribas 2003), whereas the late $\mathrm{M}$ dwarfs in CM Dra appeared to be in agreement with theoretical models (Metcalfe et al. 1996). Subsequent M dwarf binary studies appear to have corroborated this effect for mid- and early $\mathrm{M}$ dwarfs, while finding that the late $\mathrm{M}$ dwarfs may also be inflated at the few percent level (e.g.,Torres 2013; Zhou et al. 2014; Dittmann et al. 2017, and references therein). These stars also appear to have a temperature discrepancy, whereby the measured effective temperatures are lower than predicted by the models. While it has been speculated that these discrepancies may be due to strong magnetic fields inhibiting convection in the atmospheres of these stars (e.g., Chabrier et al. 2007; MacDonald \& Mullan 2012), no clear observational distinction in radii has been found between stars expected to have strong magnetic activity and stars expected to be less active ${ }^{10}$ (e.g., Doyle et al. 2011; Irwin et al. 2011). Other suggestions include the possibility

\footnotetext{
${ }^{10}$ One caveat is that the magnetic activity is not measured directly, but rather is assumed to correlate with the rotation period of the star. The latter is measured in some cases, or else assumed to be tidally synchronized to the orbital period for short-period systems.
}

that the stars have a range of compositions (e.g., Burrows et al. 2011, who consider the case of brown dwarfs and very low mass stars), that a proper equation of state is not used in generating the models (e.g., Dotter et al. 2008), or that there are systematic errors in the measurements due, for example, to spots on the stars (Morales et al. 2010; Windmiller et al. 2010).

In this paper we report the discovery by the HATNet survey (Bakos et al. 2004) of a detached, double-lined M dwarf binary with total eclipses. As an M dwarf system this object is useful for testing theoretical models of low-mass stars. Moreover, we take advantage of the total eclipses to obtain an uncontaminated near-infrared (NIR) spectrum of the primary star and a disentangled spectrum of the secondary star, which we use to measure their atmospheric parameters. The eclipse totality also allows us to obtain accurate mass and radius measurements for the system, with systematic errors due to unaccounted-for starspots that are lower than if the system were grazing. Finally, because the system was observed by the NASA $K 2$ mission, we are able to determine precise photometric parameters for the binary system, including a direct measurement of the rotation period for the primary star, which we find to be close to, but clearly not synchronized with, the orbital period of the system.

\section{Observations and Reductions}

\subsection{Photometric Detection}

HAT-TR-318-007 ${ }^{11}$ (also known as 2MASS J08503296 +1208239, Cl NGC 2682 FBC 6558, GSC2.3 N8X5006832, SDSS J085032.94+120822.8, KIC 6651， 1WGA J0850.5 $+1208 ; \quad \alpha=08^{\mathrm{h}} 50^{\mathrm{m}} 32$. $9578, \quad \delta=+12^{\circ} 08^{\prime} 23$. $^{\prime \prime} 644 ; \quad \mathrm{J} 2000$; $K=11.131 \pm 0.020 \mathrm{mag}$ ) was initially detected as a candidate transiting planet system by the HATNet survey. The available HATNet observations of this system are summarized in Table 1, while the combined phase-folded light curve is shown in Figure 1. The data are provided in Table 2.

The HATNet images were processed and reduced to trendfiltered light curves following the procedure described by Bakos

\footnotetext{
11 Following the convention established in Beatty et al. (2007), we adopt the HAT transit candidate identification as the name for this object. Here 318 indicates that the object falls in HAT field 318, while 007 indicates that this was the seventh transiting planet candidate identified in this field.
} 

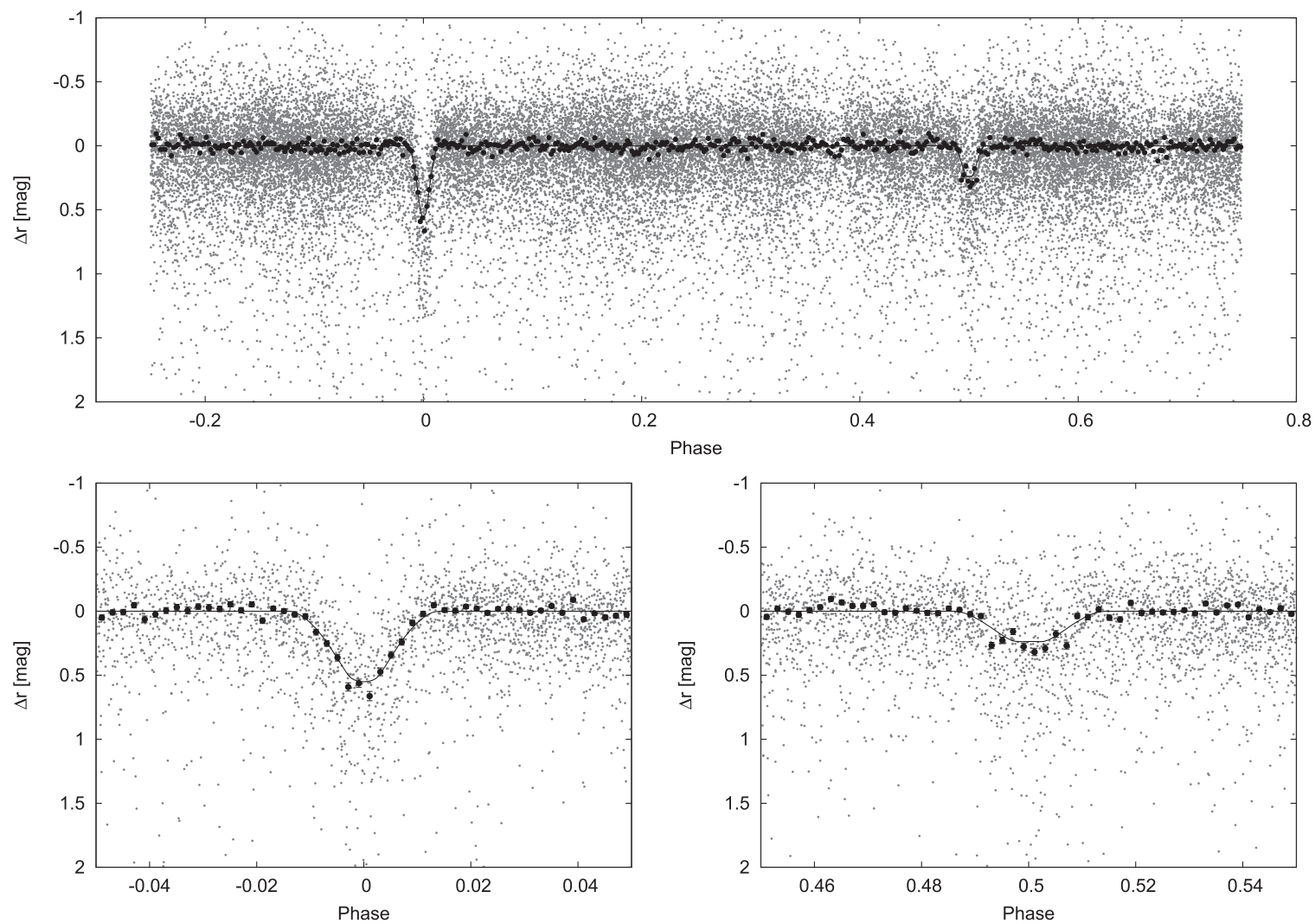

Figure 1. Phase-folded HATNet light curve for HAT-TR-318-007 after correcting for the artificial reduction in the eclipse depth due to an incorrect reference flux and application of EPD and TFA in signal-search mode. The gray filled circles are the individual measurements after application of EPD and TFA, and with the fitted-for dilution removed. The solid line shows the best-fit model. The black filled circles show the light curve binned in phase, with a bin size of 0.002 . The top panel shows the full phased light curve; the bottom left and bottom right panels show the light curve zoomed in on the primary and secondary eclipses, respectively. We show the depth-corrected light curves so that all of the HATNet data can be presented together on the same scale. The fitting itself uses the original EPD- and TFA-corrected light curves, with the additional depth correction determined as part of the fit. Note that the procedure of depth-correcting the light curves for display in this figure results in asymmetric magnitude uncertainties that distort slightly the binned light curve, as well as a slight discrepancy between the model and binned values in the figure.

et al. (2010). Candidate transits were identified using the boxfitting least-squares (BLS; Kovács et al. 2002) procedure. Typically eclipsing binary systems that show unequal primary and secondary eclipse depths are automatically rejected; however, due to the faintness of HAT-TR-318-007, the difference in eclipse depths for this star could not be clearly detected in the HATNet observations. Moreover, an inaccurate reference flux estimate for this star in our image subtraction procedure caused us to grossly underestimate the eclipse depths in some of our light curves. Subsequent photometric follow-up observations discussed in Section 2.2 showed clearly that HAT-TR-318-007 is not a transiting planet system, but also revealed the total secondary eclipses, which motivated us to continue studying this object.

Coincidentally, HAT-TR-318-007 has a projected separation on the sky of $23^{\prime}$ from the center of the open cluster M67. Although this is within the projected tidal radius of the cluster, the apparent magnitude of HAT-TR-318-007 indicates that it is clearly a foreground object and not a cluster member. Nonetheless, its location on the sky has resulted in this star having calibrated photometry from several surveys of this very well studied open cluster. Table 3 lists the available photometric measurements of the system from the literature.

\subsection{Ground-based Photometric Follow-up}

Additional photometric light curves of HAT-TR-318-007 covering both primary and secondary eclipses were obtained with the KeplerCam instrument on the FLWO $1.2 \mathrm{~m}$ telescope and the CCD imager on the Byrne Observatory at Sedgewick, CA (BOS) $0.8 \mathrm{~m}$ telescope. The dates, number of observations gathered, exposure times, and filters used are listed in Table 1. The light curves covering primary eclipse are shown in Figure 2, while those covering secondary eclipse are shown in Figure 3. These observations were reduced to light curves via the aperture photometry procedure described by Bakos et al. (2010). As discussed in Section 3.2, we applied trend filtering to the light curves simultaneously with the fitting procedure.

As seen in Figure 2, we obtained two light curves covering primary eclipse on the same night with different facilities. Both of these light curves show a slight brightening in the residuals at the same phase during egress. This is due to either a stellar flare or spots on the primary star. A similar feature is seen during egress of a secondary eclipse observed on the night of 2011 December 29 (Figure 3), but no such feature is seen during a secondary eclipse observed 274 days earlier. Note that if these features are due to spots we would not expect them to reappear at the same phase after 274 days owing to the slight difference between the stellar rotation period(s) and the orbital period of the system (Section 3.1).

\subsection{K2 Photometry}

Due to its propitious location in the field of the open cluster M67, HAT-TR-318-007 was observed by the NASA $K 2$ 
Table 2

Time-series Photometry Data for HAT-TR-318-007

\begin{tabular}{|c|c|c|c|c|c|c|c|c|c|c|}
\hline Object $^{\mathrm{a}}$ & Facility $^{\mathrm{b}}$ & Filter & BJD $-2,454,000^{\mathrm{c}}$ & Raw Mag. ${ }^{\mathrm{d}}$ & Err. Mag. & Corr. Mag. ${ }^{\mathrm{e}}$ & $\begin{array}{c}T-T_{c}^{\mathrm{f}} \\
\text { (days) }\end{array}$ & $S^{\mathrm{g}}$ & $D^{\mathrm{g}}$ & $K^{\mathrm{g}}$ \\
\hline HTR318-007 & bos & $i$ & 1930.780201 & 14.3763 & 0.0146 & 14.3523 & -0.08311 & 0.26320 & -0.00660 & 0.00930 \\
\hline HTR318-007 & bos & $i$ & 1930.781811 & 14.3679 & 0.0128 & 14.3544 & -0.08150 & 0.29080 & 0.00310 & 0.00480 \\
\hline HTR318-007 & bos & $i$ & 1930.783431 & 14.3831 & 0.0133 & 14.3657 & -0.07988 & 0.27920 & -0.00070 & 0.01190 \\
\hline HTR318-007 & bos & $i$ & 1930.785041 & 14.3746 & 0.0135 & 14.3619 & -0.07827 & 0.33410 & 0.00050 & 0.01330 \\
\hline HTR318-007 & bos & $i$ & 1930.786671 & 14.3396 & 0.0147 & 14.3339 & -0.07664 & 0.33640 & 0.00420 & 0.01530 \\
\hline HTR318-007 & bos & $i$ & 1930.788321 & 14.3473 & 0.0132 & 14.3419 & -0.07499 & 0.33640 & -0.00140 & -0.00080 \\
\hline HTR318-007 & bos & $i$ & 1930.789941 & 14.3662 & 0.0144 & 14.3549 & -0.07337 & 0.32710 & -0.00920 & -0.00830 \\
\hline HTR318-007 & bos & $i$ & 1930.791561 & 14.3643 & 0.0142 & 14.3544 & -0.07175 & 0.29770 & -0.00650 & 0.01090 \\
\hline HTR318-007 & bos & $i$ & 1930.793201 & 14.3588 & 0.0132 & 14.3453 & -0.07011 & 0.38650 & -0.01590 & 0.01060 \\
\hline HTR318-007 & bos & $i$ & 1930.794831 & 14.3753 & 0.0119 & 14.3533 & -0.06848 & 0.33170 & -0.00660 & 0.01190 \\
\hline
\end{tabular}

Notes.

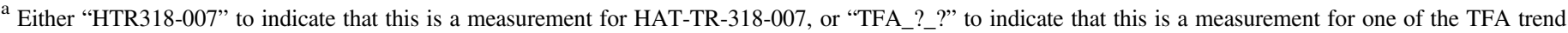

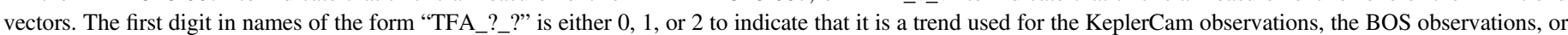

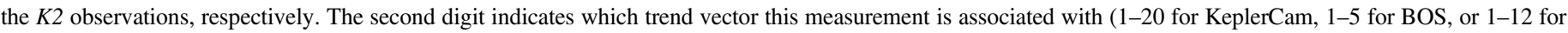

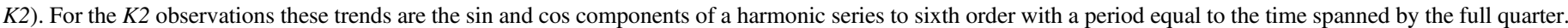

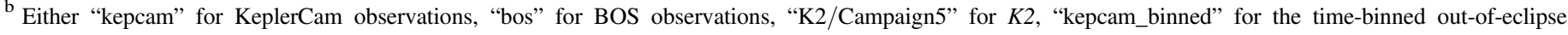

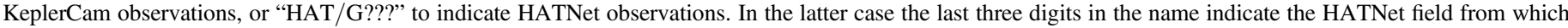
these observations were obtained. The $K 2$ observations have been cleaned as described in Section 2.3.

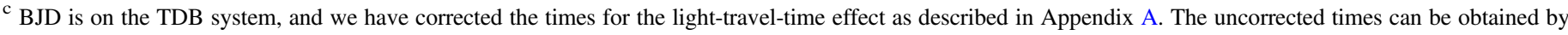
request to the authors.

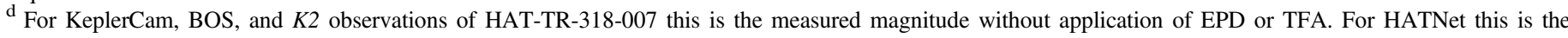
magnitude after application of EPD and TFA run in signal-search mode. For TFA trend measurements this is the value of the TFA vector at the specified time.

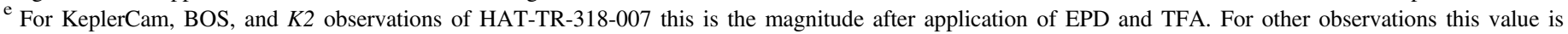
undefined.

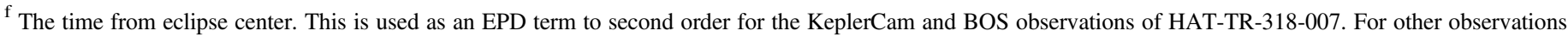
this value is left undefined.

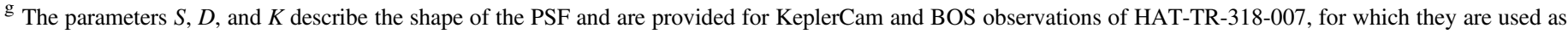

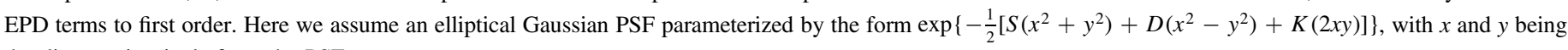
the distance in pixels from the PSF center.

(This table is available in its entirety in machine-readable form.)

mission (Howell et al. 2014) during Campaign 5. A total of 3298 long-cadence (29.4-minute) photometric time-series observations were collected by the satellite for this source (EPIC 211432946). The observations span 74.8 days from UT 2015 April 26 to UT 2015 July 10 and cover 20 primary eclipse events and 23 secondary events.

Based on the $K 2$ observations, Barros et al. (2016) have also independently identified EPIC 211432946 as an eclipsing binary system. They included it in a catalog of eclipsing binary candidates providing the period, epoch of eclipse, primary eclipse depth, eclipse and ingress durations, and flags indicating that there is a definite secondary eclipse present in the data. There is no additional analysis or discussion of this particular object in that publication, and we did not become aware of this independent identification until very shortly before submitting this paper for publication.

We considered three different publicly available reductions of the K2 long-cadence observations of HAT-TR-318-007. These include (1) the Data Release 7 K2 long-cadence light curve for HAT-TR-318-007 from the Mikulski Archive for Space Telescopes (MAST), (2) the EVEREST pixel-level decorrelated light curve from Luger et al. (2017), and (3) the decorrelated light curve produced by the method of Vanderburg \& Johnson (2014). We found that the EVEREST reduction of HAT-TR-318-007 had substantially lower scatter around the astrophysical signal and fewer residual systematic variations associated with the $6 \mathrm{hr}$ spacecraft roll than the other reductions, and so we adopt the EVEREST light curve for the remainder of the analysis.

Figure 4 shows the original EVEREST light curve of HATTR-318-007 in units of flux. In addition to the numerous primary and secondary eclipses visible, there is a periodic variation in the out-of-eclipse light curve that we associate with the rotation of one of the binary components. There is also a significant increasing trend in the flux over the quarter. The trend is correlated with the change in the zodiacal background flux over the quarter and likely indicates a slight systematic error in subtracting this background from the observations of this faint source. Because both the primary and secondary eclipse depths remain constant in flux over the quarter, this is clearly a change in the background flux level, rather than a variation in the brightness of one or more components in the system. To correct for this, we fit the out-of-eclipse points in the flux light curve as a combination of two harmonic series. The first is a sequence of six harmonics with a fundamental period of $P=3.4164940808$ days, the highest peak in the Lomb-Scargle periodogram (we discuss the photometric rotational variation seen in the $K 2$ light curve in greater detail in Section 3.1). This series captures the variation due to the periodic rotation of one of the binary components. The second series that we used was a series of six harmonics with a fundamental period of $P=93$ days, which we use to capture the variation in the background flux. We subtract the latter model from the full light curve and convert the corrected fluxes to magnitudes. The choice of $P=93$ days for the background 
Table 3

Photometric Measurements of HAT-TR-318-007 from the Literature

\begin{tabular}{lrl}
\hline \hline Filter & \multicolumn{1}{r}{ Measurement } & \multicolumn{1}{c}{ References } \\
\hline$g$ & 17.262 & KIC (Brown et al. 2011) \\
$r$ & 15.780 & KIC \\
$i$ & 14.417 & KIC \\
$z$ & 13.510 & KIC \\
DDO-51 & 16.881 & KIC \\
$J$ & $12.028 \pm 0.021$ & 2MASS (Skrutskie et al. 2006) \\
$H$ & $11.429 \pm 0.022$ & 2MASS \\
$K_{S}$ & $11.131 \pm 0.020$ & 2MASS \\
$u$ & $19.522 \pm 0.039$ & SDSS DR9 (Ahn et al. 2012) \\
$g$ & $17.270 \pm 0.004$ & SDSS \\
$r$ & $15.848 \pm 0.003$ & SDSS \\
$i$ & $14.353 \pm 0.003$ & SDSS \\
$z$ & $13.520 \pm 0.003$ & SDSS \\
3890 & $18.845 \pm 0.085$ & BATC (Fan et al. 1996) \\
5795 & $16.093 \pm 0.016$ & BATC \\
6075 & $16.118 \pm 0.017$ & BATC \\
6660 & $15.163 \pm 0.017$ & BATC \\
7215 & $14.626 \pm 0.015$ & BATC \\
8020 & $13.928 \pm 0.010$ & BATC \\
8480 & $13.784 \pm 0.015$ & BATC \\
9190 & $13.257 \pm 0.014$ & BATC \\
9745 & $13.109 \pm 0.010$ & BATC \\
NUV & $21.57 \pm 0.38$ & GALEX DR5 (Bianchi et al. 2011) \\
W1 & $11.042 \pm 0.023$ & WISE (Wright et al. 2010) \\
W2 & $10.866 \pm 0.021$ & WISE \\
W3 & $10.919 \pm 0.106$ & WISE \\
W4 & $<8.853$ & WISE \\
$B$ & 17.450 & NOMAD (Zacharias et al. 2004) \\
$V$ & 16.130 & NOMAD \\
$R$ & 15.260 & NOMAD \\
$G$ & $14.922 \pm 0.020$ & Gaia DR1 (Gaia Collaboration et al. 2016) \\
\hline & &
\end{tabular}

Notes.

${ }^{\text {a }}$ Observations of M67 were carried out as part of calibrating the KIC. HATTR-318-007 was serendipitously included in these observations.

${ }^{\mathrm{b}}$ Data from the BATC color survey of M67. Values are AB magnitudes within narrowband filters. The filter name is the center wavelength of the filter in angstroms.

harmonic series is effectively arbitrary. This value is longer than the time span of the $K 2$ data and was determined by optimizing a fit during a preliminary analysis of the light curve, but was kept fixed during our final analysis.

Upon further inspection, we found that there are numerous short-timescale brightenings in the light curve that may either be due to flaring activity or result from imperfect removal of systematic artifacts associated with the spacecraft roll for this large-amplitude variable star. To deal with these, we clipped out-of-eclipse points with residuals less than -0.005 mag from a first-iteration model fit to the full light curve. We also restrict the analysis to observations obtained after BJD $>2,457,145$ to avoid large systematic variations seen in the residuals at the start of the time series.

Figure 5 shows the resulting corrected $K 2$ light curve together with our best-fit physical model (Section 3.2). Figure 6 shows the phase-folded $K 2$ light curve with our full model for the rotational signals of the two component stars subtracted and zoomed in on the primary and secondary eclipses, respectively. In these same figures we overplot our best-fit model for the eclipse signals, accounting for the $1625.35 \mathrm{~s} \mathrm{K2}$ integration time.

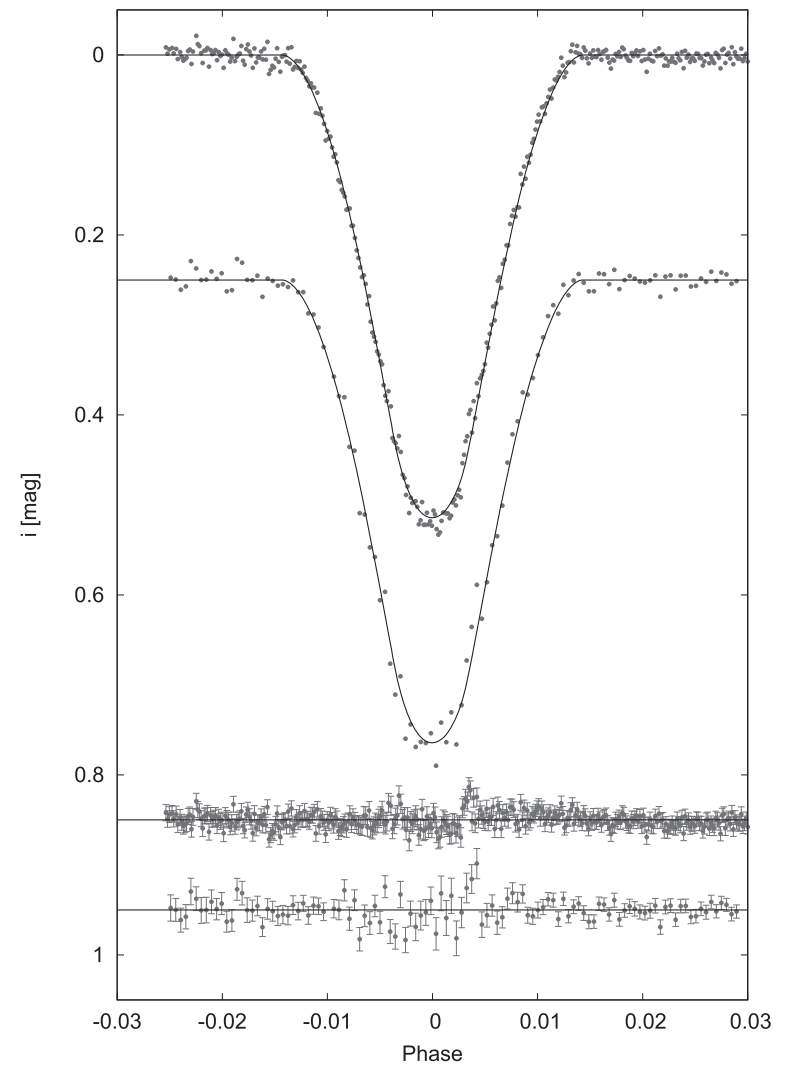

Figure 2. Primary eclipse light curves for HAT-TR-318-007 offset vertically for clarity. The top light curve was obtained with KeplerCam on the night of 2012 January 3, while the second light curve was obtained with BOS on the night of 2012 January 3 . The solid lines show the best-fit model light curves. Residuals from the best-fit models are shown at the bottom, in the same order as above.

\subsection{Spectroscopic Observations}

\subsubsection{Optical}

We obtained optical spectra of HAT-TR-318-007 using the Tillinghast Reflector Echelle Spectrograph (TRES), with the medium-resolution fiber, on the $1.5 \mathrm{~m}$ Tillinghast Reflector at FLWO (Fúrész 2008). This instrument and configuration deliver multiorder spectra with a resolution of $\lambda / \Delta \lambda \approx 44,000$ and a wavelength coverage of $\sim 3900-8900 \AA$. A total of 14 spectra were obtained between 2011 March 28 and 2011 December 9. The spectra were extracted and wavelength-calibrated using the pipeline of Buchhave et al. (2010). While the star is too faint for much of the optical spectra to be useful, we clearly detected double $\mathrm{H} \alpha$ emission lines moving in phase with the photometric ephemeris, as well as TiO absorption bands from both components. The strength of the $\mathrm{H} \alpha$ emission line for the primary star appears to vary by $\sim 20 \%$ between observations. We used these observations to measure RVs for both of the components with the two-dimensional correlation (TODCOR; Zucker \& Mazeh 1994) algorithm, using a TRES spectrum of Barnard's star as a template for both components. The correlation was done over a single order containing TiO lines spanning 7063-7201 ̊. In doing this, we determine an optical spectroscopic light ratio of $L_{\mathrm{B}} / L_{\mathrm{A}}=0.350 \pm 0.014$ from the highest signal-to-noise ratio $(\mathrm{S} / \mathrm{N})$ exposures with unblended lines, and we used this value in measuring the velocities from all of the spectra. This is similar to the light ratio of $L_{B, i} / L_{A, i}=0.300 \pm 0.005$ determined from the $i$-band light curves. Because the $i$ bandpass is broader than the 


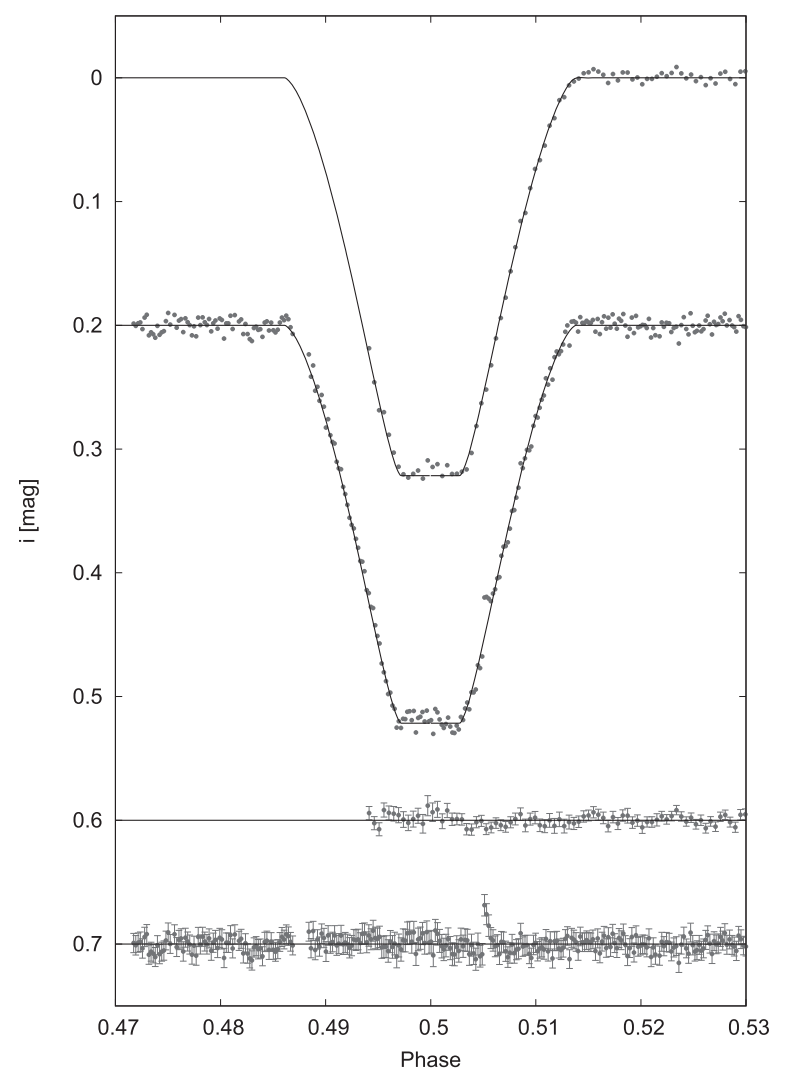

Figure 3. Secondary eclipse light curves for HAT-TR-318-007 offset vertically for clarity. Both light curves were obtained with KeplerCam, the top on the night of 2011 March 30, the second on the night of 2011 December 29. Residuals from the best-fit models are shown at the bottom, in the same order as above.

order over which the spectroscopic light ratio is determined, we do not expect these two estimates to be equal. The resulting RVs, measured relative to Barnard's star, are listed in Table 4 and plotted in Figure 7.

\subsubsection{NIR}

In order to determine the atmospheric parameters for the individual components of HAT-TR-318-007, we obtained medium-resolution NIR spectra using the Folded-port InfraRed Echellette (FIRE) spectrograph (Simcoe et al. 2013) on the 6.5 m Magellan Baade telescope at Las Campanas Observatory in Chile. Observations were conducted during the last $\sim 4 \mathrm{hr}$ before twilight on the UT nights of 2011 December 09, 10, and 11 , with a total secondary eclipse occurring during the night of 2011 December 10. We observed HAT-TR-318-007 continuously over an 83-minute period encompassing the secondary eclipse and on each of the nights before and after the eclipse. For calibration we also observed a number of $\mathrm{M}$ dwarf standard stars. Our observations are summarized in Table 5. In addition to the stars listed therein, we also observed GJ 273 and GJ 382; however, due to a poor telluric correction, the data for these two stars proved to be not usable.

Observations were performed in echelle mode using a 0 ". 6 slit width ( 7 "length) with readout performed in the Fowler 1 mode. This setup provides spectra with a resolution of $\lambda / \Delta \lambda=6000$ over the $0.82-2.51 \mu \mathrm{m}$ wavelength range. To facilitate sky subtraction and flux calibration, we performed A-B nodding and observed telluric standards near in time and
Table 4

Relative Radial Velocities for HAT-TR-318-007 from FLWO 1.5 m/TRES

\begin{tabular}{cccccc}
\hline \hline BJD & $\begin{array}{c}\mathrm{RV}_{\mathrm{A}}{ }^{\mathrm{a}} \\
\left(\mathrm{km} \mathrm{s}^{-1}\right)\end{array}$ & $\begin{array}{c}\sigma \mathrm{RV}_{\mathrm{A}}{ }^{\mathrm{b}} \\
\left(\mathrm{km} \mathrm{s}^{-1}\right)\end{array}$ & $\begin{array}{c}\mathrm{RV}_{\mathrm{B}}{ }^{\mathrm{a}} \\
\left(\mathrm{km} \mathrm{s}^{-1}\right)\end{array}$ & $\begin{array}{c}\sigma \mathrm{RV}_{\mathrm{B}}{ }^{\mathrm{c}} \\
\left(\mathrm{km} \mathrm{s}^{-1}\right)\end{array}$ & $\mathrm{C}^{\mathrm{d}}$ \\
\hline $2,455,648.7452$ & 177.85 & 0.66 & 79.52 & 1.77 & 0.714 \\
$2,455,662.6386$ & 186.50 & 0.76 & 65.06 & 2.03 & 0.623 \\
$2,455,667.6664$ & 93.06 & 0.79 & 216.39 & 2.08 & 0.608 \\
$2,455,668.6728$ & 167.85 & 1.00 & 90.28 & 2.66 & 0.476 \\
$2,455,672.6665$ & 186.85 & 0.66 & 62.07 & 1.76 & 0.718 \\
$2,455,699.6407$ & 177.31 & 0.86 & 81.83 & 2.28 & 0.554 \\
$2,455,704.6528$ & 102.01 & 0.71 & 203.50 & 1.90 & 0.668 \\
$2,455,888.9826$ & 134.62 & 0.64 & 151.02 & 1.69 & 0.747 \\
$2,455,899.9911$ & 187.85 & 0.96 & 64.69 & 2.58 & 0.492 \\
$2,455,900.9678$ & 122.51 & 1.39 & 172.33 & 3.69 & 0.343 \\
$2,455,901.9759$ & 104.17 & 0.87 & 199.50 & 2.32 & 0.545 \\
$2,455,902.9955$ & 182.46 & 0.78 & 68.80 & 2.06 & 0.614 \\
$2,455,903.9885$ & 151.06 & 0.60 & $\ldots$ & $\ldots$ & 0.792 \\
$2,455,904.9681$ & 91.86 & 0.63 & 220.20 & 1.68 & 0.750 \\
\hline
\end{tabular}

Notes.

a RVs are measured relative to Barnard's star.

${ }^{\mathrm{b}}$ Primary star RV uncertainties have been scaled by a factor of 0.81 as determined in Section 3.2.

${ }^{c}$ Secondary star RV uncertainties have been scaled by a factor of 0.96 as determined in Section 3.2.

d Normalized cross-correlation peak height.

e This observation was obtained near eclipse. A separate velocity for the secondary component could not be resolved from the primary velocity.

airmass to each of the targets. To determine the wavelength calibration, we obtained ThAr lamp spectra before or after a set of observations for a given science target. We also obtained quartz lamp spectra and observations of the twilight sky to use in tracing the echelle orders and creating a flat field.

The observations were reduced to flux-calibrated spectra using the FIRE reduction pipeline (Simcoe et al. 2013) downloaded in 2012 May. The reduction was performed using the boxcar extraction mode, with apertures determined automatically, and with the closest associated B (A) nod used to determine the sky subtraction for a given A (B) nod observation.

To extract separate spectra for the primary and secondary star components of HAT-TR-318-007, we performed Fourier-based spectral disentangling using version 3 of the FDBinary program (Ilijic et al. 2004). We fixed the orbital parameters to those determined from fitting the TRES observations, as the FIRE observations had poor phase coverage, leading to a poor constraint on the parameters. We interpolated all spectra of HAT-TR318-007 to a common wavelength grid uniformly sampled in $\log \lambda$, linearly interpolating over the wavelength ranges $0.9332-0.9378 \mu \mathrm{m}, 1.1125-1.1371 \mu \mathrm{m}, 1.343967-1.48614 \mu \mathrm{m}$, $1.7990619-1.9427 \mu \mathrm{m}, \quad 1.9667-1.97 \mu \mathrm{m}, \quad 2.4364-2.4401 \mu \mathrm{m}$, $2.4473-2.4601 \mu \mathrm{m}, 2.4663-2.4695 \mu \mathrm{m}$, and $2.4777-2.48 \mu \mathrm{m}$, which have significant telluric contamination. We performed the disentangling separately for the $(Z+Y)-, J-, H$-, and $K$-band regions of the spectra (for the purposes of this paper, these regions correspond to wavelength ranges of $0.832-1.112 \mu \mathrm{m}$, $1.137-1.344 \mu \mathrm{m}, 1.486-1.798 \mu \mathrm{m}$, and 1.943-2.414 $\mu \mathrm{m}$, respectively). We excluded spectra obtained during partial eclipse.

The resulting spectra for both the primary and secondary components are shown in Figure 8, together with theoretical templates, which are described further in Appendix C. These spectra are also shown at higher resolution in Figures 9 and 10. The data are provided in Table 6. 

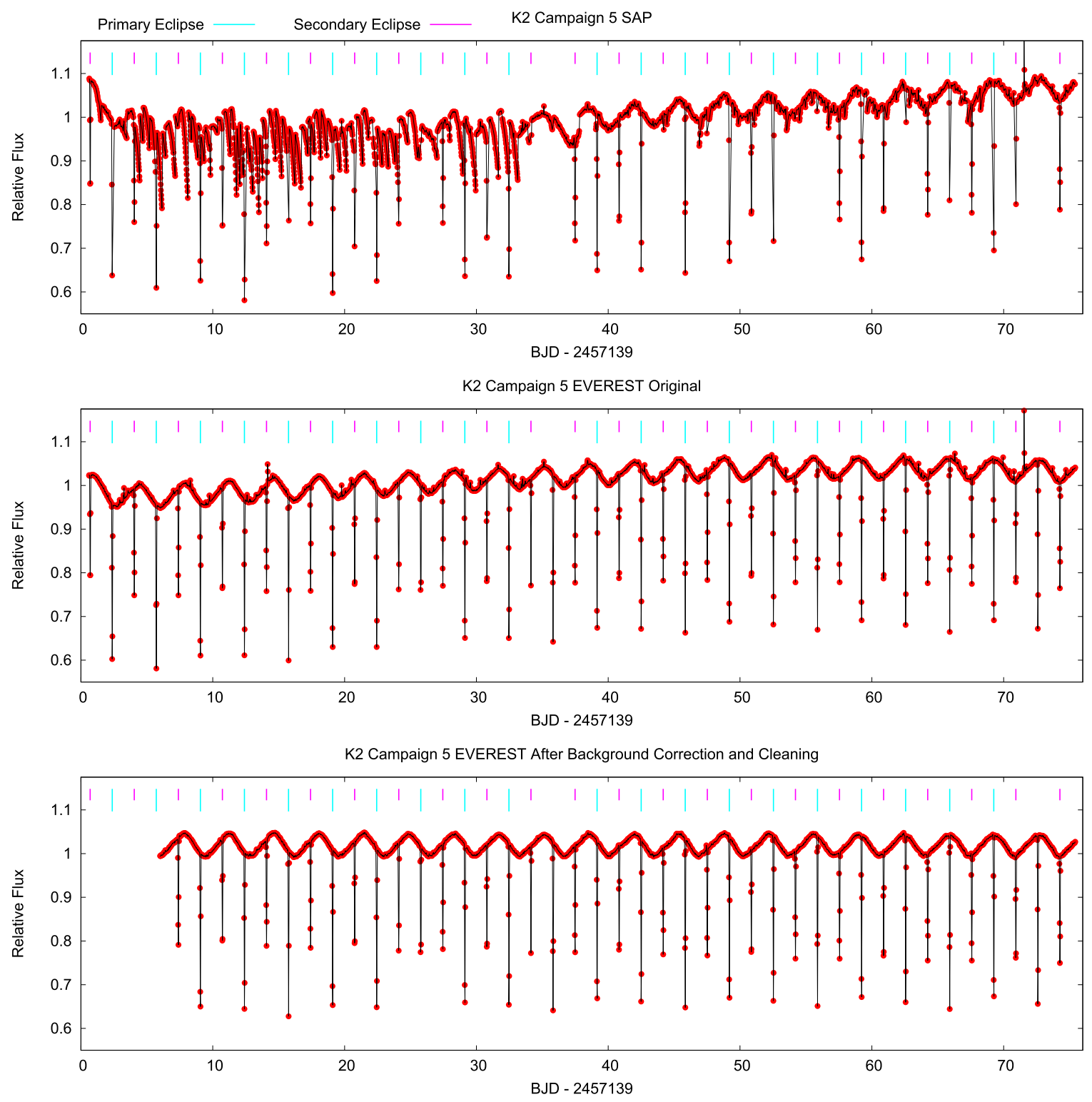

Figure 4. K2 Campaign 5 light curve of HAT-TR-318-007 (EPIC 211432946) showing the original SAP light curve (top); the EVEREST light curve from Luger et al. (2017), which applies a pixel-level decorrelation against the spacecraft roll (middle); and the EVEREST light curve after correcting for an increase in the brightness (which we attribute to a slight error in the background correction over the course of the campaign), filtering of bright outliers (likely due to stellar flares), and exclusion of points from the start of the campaign (bottom). This is the light curve that we analyze in Section 3.2. In all cases the overplotted line is not a model, but simply connects observed points in time. Observed primary and secondary eclipse events are marked in each panel.

Table 5

Summary of FIRE/Magellan Observations

\begin{tabular}{|c|c|c|c|c|c|c|}
\hline Object & $\begin{array}{c}\text { Date(s) } \\
(2011-12-\#)\end{array}$ & $K_{s} \mathrm{mag}$ & Spectral Type & Nspectra & $\begin{array}{c}\text { Mean } \\
\text { Exp. Time }\end{array}$ & Total S/N \\
\hline HAT-TR-318-007 & $09,10,11$ & 11.13 & M3.6+M5.0 & 21 & 371.4 & 490.2 \\
\hline GJ 205 & 11 & 4.04 & M1.5 & 2 & 1.0 & 169.1 \\
\hline GJ $250 \mathrm{~B}$ & 09 & 5.72 & M2.5 & 2 & 1.5 & 204.4 \\
\hline GJ 352 & 10,11 & 5.51 & M3.0 & 3 & 1.0 & 191.9 \\
\hline GJ 285 & 09 & 5.70 & M4.0 & 2 & 1.0 & 224.8 \\
\hline GJ 3348 B & 11 & 8.79 & M4.5 & 2 & 60.0 & 146.1 \\
\hline GJ 283 & 10 & 9.29 & M6.0 & 2 & 60.0 & 161.4 \\
\hline NLTT 15867 & 11 & 10.31 & M6.0 & 2 & 225.0 & 178.6 \\
\hline LHS 2065 & 10 & 9.94 & M9.0 & 2 & 180.0 & 166.8 \\
\hline
\end{tabular}



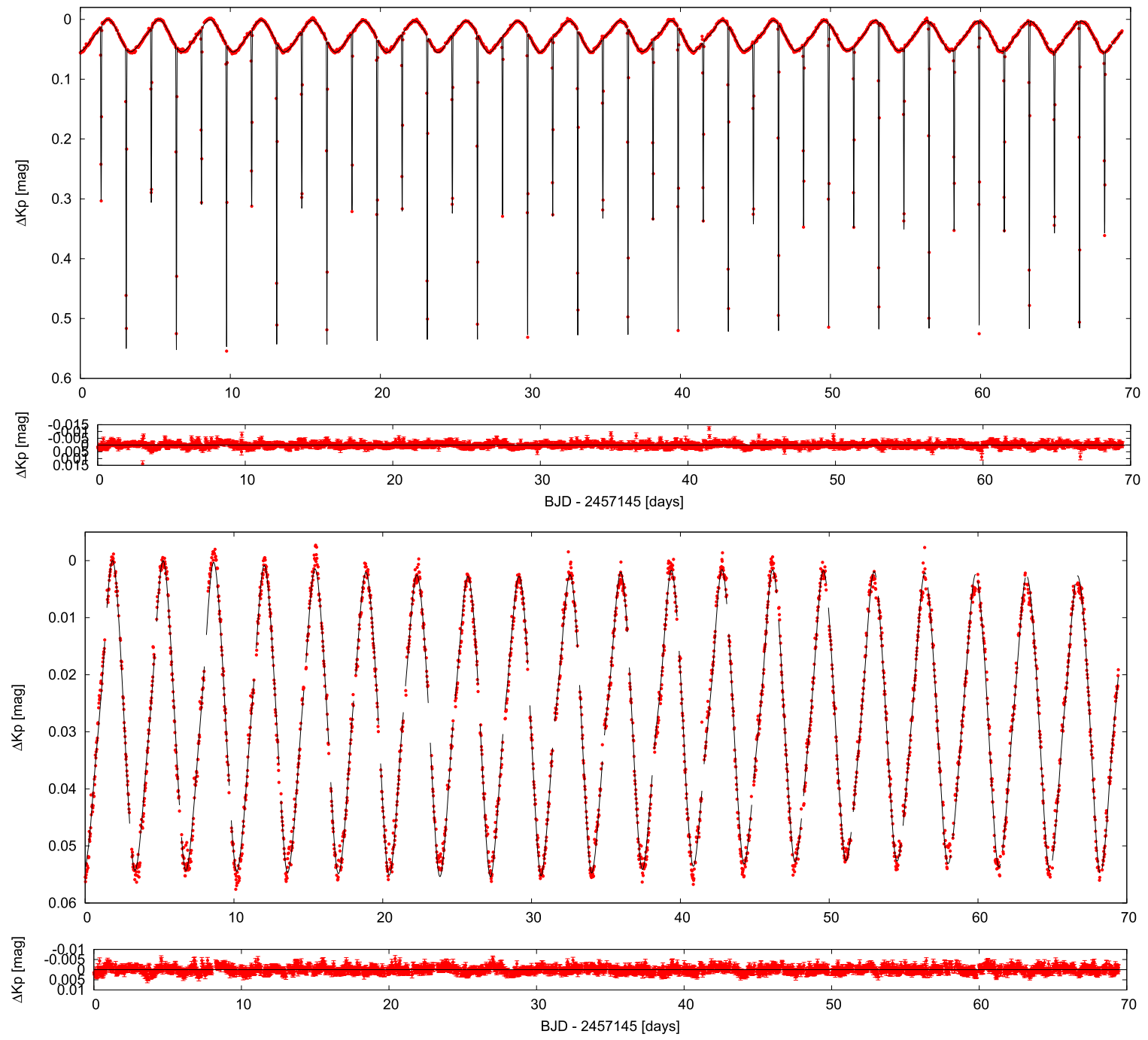

Figure 5. Top: $K 2$ Campaign 5 light curve of HAT-TR-318-007 showing the EVEREST light curve after correcting for a systematic variation in the background flux and filtering outliers. Overplotted is our best-fit model including primary and secondary eclipses, ellipsoidal variability (which is included through the JKTEBOP model), and the $P=3.41315_{-0.00032}^{+0.00030}$ day and $P=3.28498_{-0.00034}^{+0.00035}$ day rotational variation attributed in this modeling to the primary and secondary components, respectively. The residuals from the best-fit model are shown immediately below the full light curve. Bottom: same as the top panel, but here we exclude points within 0.02 in phase from the primary and secondary eclipse centers to highlight the out-of-eclipse variation and its phase coherence over the time span of the observations.

\section{Analysis}

\subsection{Stellar Rotation and Activity}

We use the EVEREST K2 light curve of HAT-TR-318-007 to characterize the photometric variability of the system due to stellar activity. The light curve shows a clear sinusoidal variation with a period of $3.41315_{-0.00032}^{+0.00030}$ days, as detected by the Generalized Lomb-Scargle (GLS) periodogram (Zechmeister \& Kürster 2009). The value and uncertainty listed are based on our full analysis of the system including rotational variability as discussed in Section 3.2. The signal remains coherent over the full 74.8-day time span. Such a long spot coherence timescale is typical of $\mathrm{M}$ dwarf stars with large photometric amplitudes. Based on Figure 8 of Giles et al. (2017), who used autocorrelation functions of Kepler light curves to determine the typical spot coherence lifetime as a function of spectral type and light-curve rms, an M dwarf star with a Kepler rms of $\sim 1 \%$ has an autocorrelation function that decays with an $e$-folding lifetime of $\sim 400$ days. This is significantly longer than the time span of the $K 2$ observations. Figure 11 shows the GLS periodogram of the cleaned and background-corrected $K 2$ light curve after removing points obtained during eclipses.

In order to search for other periodic signals in the light curve, we subtract the best-fit model of the $3.41315_{-0.00032}^{+0.00030}$ day signal and calculate the GLS periodogram of the residuals. We find a significant peak in the periodogram at a period of $P=1.63743194$ days (Figure 11). Inspection of the residual light curve phase-folded at both this period and double the period indicates that the double-period value is the correct period, as the two minima and maxima per cycle have noticeably different depths and heights. Based on the full analysis of Section 3.2, we find a period and uncertainty of $P=3.28498_{-0.00034}^{+0.00035}$ days for this signal.

A third iteration of GLS yields no additional significant periodic signals (Figure 11).

Although close to the orbital period of 3.344 days, the two most significant periods identified in the out-of-eclipse light curve are definitely not the same as the orbital period, as can be seen by the change in time in the rotational phase at which eclipses are observed. In order to identify which binary star component is giving rise to each of these signals, we make use 

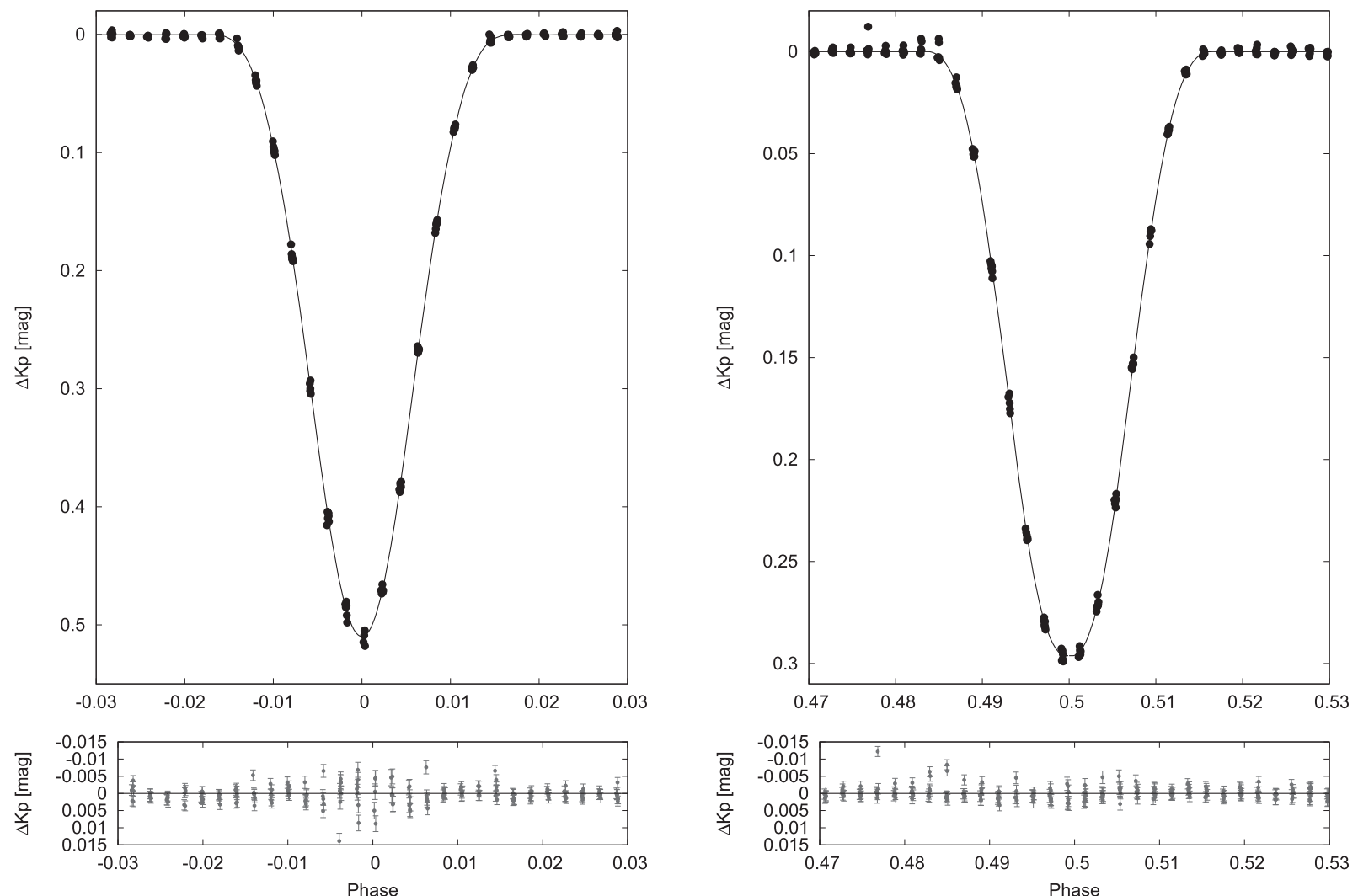

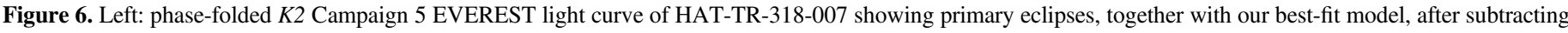

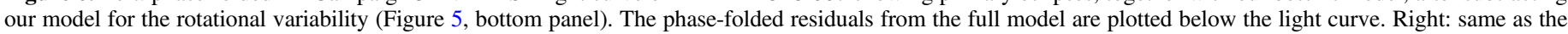
left panel, but here we show the secondary eclipses. Note the different vertical scale used in each of the panels.

of the $K 2$ observations obtained near the base of the total secondary eclipses, which enable us to cleanly separate the contributions from each component to the total integrated brightness of the system at those moments in time. We take observations having a midexposure time within 11 minutes of the center time of a secondary eclipse as providing a measurement of the integrated brightness of the primary star without any contribution from the secondary. We then subtract this flux from the average flux of nearby points that were obtained between 77 and 106 minutes from the center of the eclipse to determine the integrated brightness of the secondary star without any contribution from the primary.

In Figure 12 we show how the primary star brightness values determined directly from the total secondary eclipse observations phase up in excellent agreement with the larger-amplitude $P=3.41315_{-0.00032}^{+0.00030}$ day out-of-eclipse modulation, where we have subtracted off the average flux contribution from the secondary component in plotting the out-of-eclipse values and have converted everything to magnitudes in the plot. By contrast, the secondary-star brightness values inferred from the secondary eclipse depths do not phase up at all with this periodic out-of-eclipse variation when it is attributed to the secondary star. We conclude that this variability arises from the primary star, and we interpret the period as the photometric rotation period of this component.

Similarly, in Figure 13 we show how the secondary-star brightness values determined directly from the observed secondary eclipse depths compare with the smaller-amplitude $P=$ $3.28498_{-0.00034}^{+0.00035}$ day out-of-eclipse modulation, after subtracting the flux contribution from the primary component, including the larger-amplitude $P=3.41315_{-0.00032}^{+0.00030}$ day variation. We show the results when we attribute the variation to the primary star, comparing the directly measured primary-star brightness (after subtracting the large-amplitude $P=3.41315_{-0.00032}^{+0.00030}$ day variation) to the out-of-eclipse modulation after subtracting both the large-amplitude $P=3.41315_{-0.00032}^{+0.00030}$ day variation and a constant flux contribution from the secondary component. In this case the scatter in the few observed eclipses is too large to clearly determine which stellar component is giving rise to the $P=3.28498_{-0.00034}^{+0.00035}$ day variability. In carrying out the joint analysis (Section 3.2), we model the observations both ways, attributing the variability to the secondary component and attributing it to the primary component. We find that the two scenarios are indistinguishable based on the observations, with $\Delta \chi^{2}<1$ between the two best-fit models.

We also analyzed the HATNet and ground-based photometric follow-up observations for stellar activity signals. We fit a harmonic signal to the HATNet light curves from each of the four separate fields containing HAT-TR-318-007. In each case we removed observations obtained during eclipse before conducting the fit. Including the correction for dilution, we find the following $95 \%$ confidence upper limits on the semiamplitude of variability: $\mathrm{HAT} / \mathrm{G} 317=0.017 \mathrm{mag}$, HAT $/ \mathrm{G} 318=0.026 \mathrm{mag}$, HAT $/ \mathrm{G} 365=0.017 \mathrm{mag}$, HAT $/$ $\mathrm{G} 366=0.013 \mathrm{mag}$. A combined analysis of the four fields yields an upper limit of $\lesssim 0.004 \mathrm{mag}$ on the semiamplitude of variability at the $K 2$ period. We conclude that the signal present during the $K 2$ observations was substantially lower during the time period of the HATNet observations and/or that the photometric variations must have a significantly smaller 

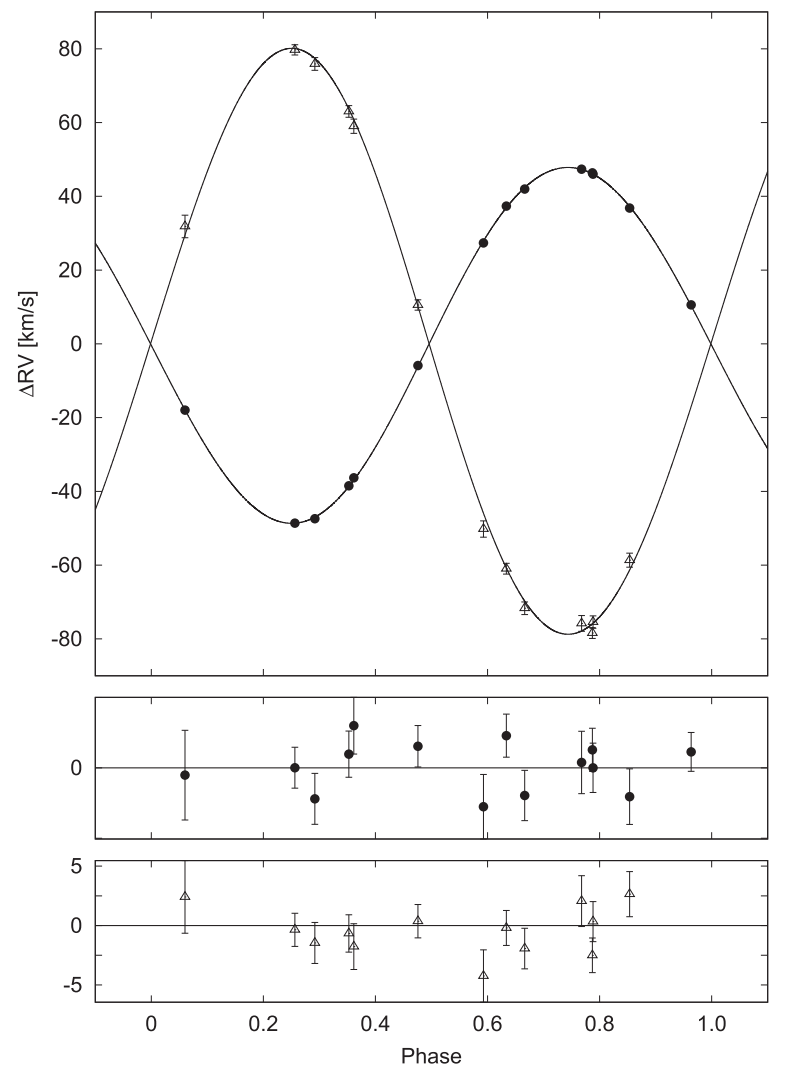

Figure 7. Phase-folded RVs for HAT-TR-318-007 measured with TRES on the FLWO $1.5 \mathrm{~m}$ together with the best-fit orbit (solid lines). Filled circles show the RVs for the primary star, while open triangles show the RVs for the secondary star. The bottom two panels show the residuals for each component from the best-fit model. The systemic $\gamma$ velocity has been subtracted from the RVs.

amplitude in $r$ than was observed by $K 2$ in the bluer Kepler bandpass.

We also checked the HATNet light curves independently for periodic and quasi-periodic variations using the Lomb-Scargle periodogram (Lomb 1976; Scargle 1982) and the discrete autocorrelation function (Edelson \& Krolik 1988). No significant signals are identified by either method. When the combined HATNet light curve is searched for periodicity via a discrete Fourier transform analysis, there appears to be a peak in the power spectrum at a period of 27.87 days. The origin of this signal is unclear, though it may be due to modulation in the background at close to the lunar period.

While the combined KeplerCam $i$-band observations have much sparser time coverage compared to the HATNet and $K 2$ observations, they have sufficient precision to detect the photometric variability in the $i$ bandpass. To do this, we first perform a combined reduction of all KeplerCam $i$-band observations, including out-of-eclipse observations made on the nights of 2012 March 02 and 2012 March 03. This reduction differs from the reduction used to make the light curves included in our joint fit, which was performed independently for each night. The independent reduction produces higher-precision light curves, but with differing magnitude zero-points for each night. Figure 14 shows the out-of-eclipse portion of the KeplerCam light curve, together with the rotational signal as determined from the $K 2$ light curves. Overall the KeplerCam $i$-band observations, which were obtained several years before the $K 2$ data, show
Table 6

Disentangled FIRE/Magellan Spectra of HAT-TR-318-007

\begin{tabular}{ccccc}
\hline \hline $\begin{array}{c}\text { Wavelength } \\
(\AA)\end{array}$ & Pri. Flux $^{\text {a }}$ & Err. Pri. Flux $^{\mathrm{b}}$ & Sec. Flux & Err. Sec. Flux \\
\hline 8321.040 & 3.44391 & 0.05910 & 0.56731 & 0.04367 \\
8321.390 & 3.45643 & 0.04704 & 0.50339 & 0.03266 \\
8321.730 & 3.46005 & 0.04686 & 0.56625 & 0.02873 \\
8322.080 & 3.48033 & 0.04144 & 0.67510 & 0.03531 \\
8322.430 & 3.55268 & 0.04595 & 0.63200 & 0.03725 \\
8322.780 & 3.47703 & 0.05433 & 0.67137 & 0.03735 \\
8323.120 & 3.38021 & 0.04889 & 0.68143 & 0.07494 \\
8323.470 & 3.40215 & 0.07842 & 0.70101 & 0.05821 \\
8323.820 & 3.39484 & 0.10027 & 0.66955 & 0.06367 \\
8324.160 & 3.36470 & 0.06003 & 0.57566 & 0.08180 \\
\hline
\end{tabular}

Notes.

a The spectra have been flux-calibrated using observations of telluric standards. The fluxes have units of power per wavelength, but are on an arbitrary scale.

${ }^{b}$ Uncertainties based on photon-counting statistics for the individual observations of HAT-TR-318-007, propagated through the spectral disentangling procedure via a Monte Carlo simulation.

(This table is available in its entirety in machine-readable form.)

significantly smaller amplitude out-of-eclipse variability than was seen in the Kepler bandpass. The variability that is seen in the KeplerCam data does not phase up with the rotational period and may be due to systematic errors in the photometry, which are fit for in modeling the individual KeplerCam eclipse observations, but which are not corrected for in the combined light curve. We conclude that the rotational variability in the $i$ band has an amplitude $\lesssim 0.01 \mathrm{mag}$. Note that as the time separation of $\sim 1200$ days between the ground-based observations and the $K 2$ observations is much longer than the typical spot coherence timescale of $\sim 400$ days for an $\mathrm{M}$ dwarf like HAT-TR-318-007A (Giles et al. 2017), it is not surprising that a coherent signal seen in the $K 2$ data is not observed in the ground-based observations.

\subsection{Joint Modeling of the Light Curves and Radial Velocity Curves}

To determine the masses and radii of the component stars of HAT-TR-318-007, we conducted a joint modeling of the RV and light curves. We used the JKTEBOP detached eclipsing binary light-curve model (Nelson \& Davis 1972; Etzel 1981; Popper \& Etzel 1981; Southworth et al. 2004a, 2004b), together with simple Keplerian orbits, to describe the RVs. The EBOP model includes the limb darkening, gravity darkening, and "reflection" effects and also accounts for the tidal distortion of the components by approximating the stars as ellipsoids. We do not include Doppler boosting in our light-curve model, as we estimate an amplitude of $\sim 0.1 \%$ (e.g., Shporer 2017), which is within the photometric noise.

To account for the starspot modulation in the light curves, we followed a method similar to that of Irwin et al. (2011), where instead of attempting to model individual spots on the surface of each component, we assume that the full surface brightness of each component varies following a harmonic series and use this to adjust the $J_{2} / J_{1}$ surface brightness ratio input to the EBOP model at each time step. 

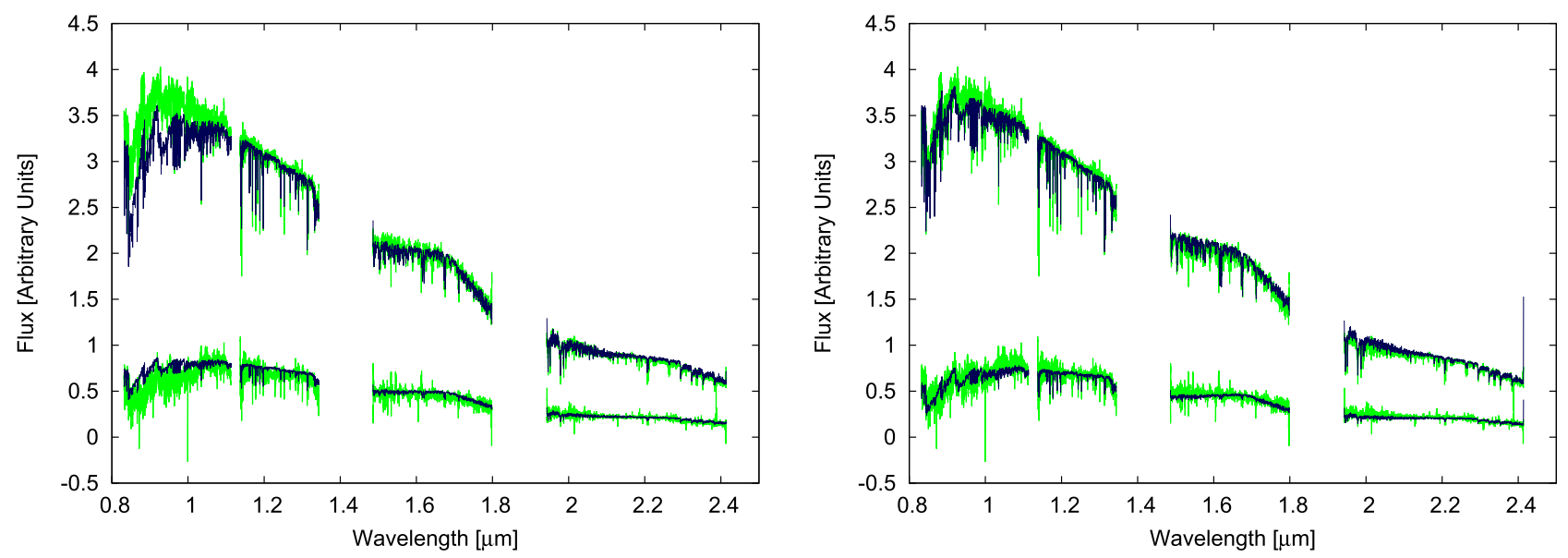

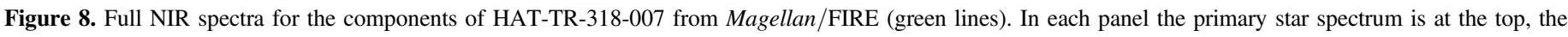

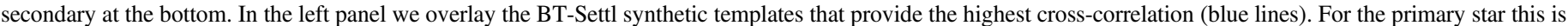

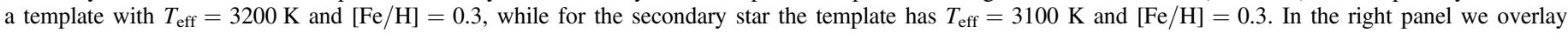

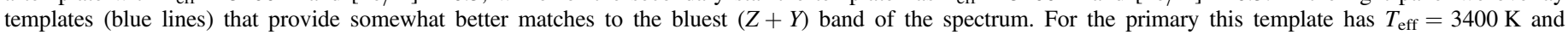
$[\mathrm{Fe} / \mathrm{H}]=0.3$, while for the secondary this has $T_{\text {eff }}=2900 \mathrm{~K}$ and $[\mathrm{Fe} / \mathrm{H}]=0.3$.

The assumed Fourier series has the form

$$
\begin{aligned}
& \Delta \operatorname{mag}_{i, j}(t)=a_{0, i, j}\left\{\cos \left[2 \pi\left(t / P_{i}+\phi_{0, i}\right)\right]\right. \\
& \left.\quad+\sum_{k=1}^{N_{\text {harm }}} b_{k, i} \cos \left[2 \pi\left(t(k+1) / P_{i}+\psi_{k, i}+(k+1) \phi_{0, i}\right)\right]\right\}
\end{aligned}
$$

and

$$
L_{i, j}(t)=L_{0, i, j} 10^{-0.4 \Delta \operatorname{mag}_{i, j}(t)}
$$

where $L_{i, j}(t)$ is the luminosity of star $i$ (primary or secondary) in filter $j$ at time $t$, and the average luminosity of the star is $L_{0, i, j}$. The free parameters in the model are the rotation period of the star $P_{i}$, the amplitude of the fundamental mode of the harmonic series $a_{0, i, j}$ in filter $j$, the phase of the fundamental mode $\phi_{0, i}$, and the relative amplitudes $b_{k, i}$ and relative phases $\psi_{k, i}$ of the higher harmonics. We adopt $N_{\text {harm }}=5$, which provides a good fit to the $K 2$ out-of-eclipse variability. Note that parameterizing it this way, using the relative amplitudes and phases for the higher harmonics, ensures that the shape of the signal is the same in all bandpasses, while the overall amplitude may vary between the bandpasses. It also has the benefit of removing the large correlation between $\phi_{0, i}$ and $\phi_{k, i}=\psi_{k, i}+(k+1) \phi_{0, i}$ that would be present if the phases rather than relative phases of the harmonics were used in the fit.

We then scale $J_{2} / J_{1}$ in filter $j$ by $L_{2, j}(t) / L_{1, j}(t)$ and adjust the stellar luminosities and third light contribution input into EBOP, for that time step, as well. Note that the limb-darkening coefficients are kept fixed at each time step in this analysis, so changes in the central surface brightness of the star lead to proportional changes in the integrated light from that source.

Our treatment differs from Irwin et al. (2011) in two ways. The first difference is our use of a higher-order Fourier series, compared to a simple sinusoidal modulation considered by Irwin et al. (2011). The higher-order series is necessary in our case to represent the more complicated shape of the $K 2$ out-ofeclipse variations seen in HAT-TR-318-007. The second difference is in the technical details of how we apply the time-varying surface brightness to the output light-curve model. Irwin et al. (2011) indicate that they modulate the out-of-eclipse and eclipsed light values computed by JKTEBOP. This yields the same result as our approach of varying the input $J_{2} / J_{1}$ values, while also adjusting the total luminosities of the components.

To allow for the possibility that some of the spotted regions on the primary are not eclipsed, we added an additional harmonic series to the flux that does not enter into the eclipse model. Namely, we use

$$
L_{\mathrm{tot}}(t)=L_{\mathrm{EBOP}}(t)+L_{3}(t)
$$

with

$$
\begin{aligned}
& L_{3}(t)=a_{0,3, j}\left\{\cos \left[2 \pi\left(t / P_{1}+\phi_{0,3}\right)\right]\right. \\
& \left.\quad+\sum_{k=1}^{N_{\text {harm }}} b_{k, 3} \cos \left[2 \pi\left(t(k+1) / P_{1}+\psi_{k, 3}+(k+1) \phi_{0,3}\right)\right]\right\}
\end{aligned}
$$

and using the primary star rotation period $P_{1}$. This is similar to the method of Irwin et al. (2011), except in this case we allow both the signal shape and amplitude of the uneclipsed modulation to be independent from the shape and amplitude of the eclipsed modulation. An additional constant third light parameter is incorporated as well into the model.

This treatment of the spot modulation clearly is simplistic, as it ignores the detailed inhomogeneous brightness distribution on the surface. However, a more detailed modeling of the spotted surfaces of the star is beyond the scope of this work. Moreover, as shown by Irwin et al. (2011), the simple model is quite flexible in describing the light-curve shape and accurately captures the resulting uncertainties in the estimated stellar radii. We also note that we are assuming here that there is no evolution in time in the dominant rotational modulation (no significant evolution is seen over the $K 2$ observations, but the $r$-band and $i$-band observations were obtained many years earlier and there may have been significant spot evolution in the intervening period). This is necessary because the highprecision $i$-band observations do not have significant out-ofeclipse phase coverage, and we are unable to determine the shape of the rotational signal independently from those observations. 

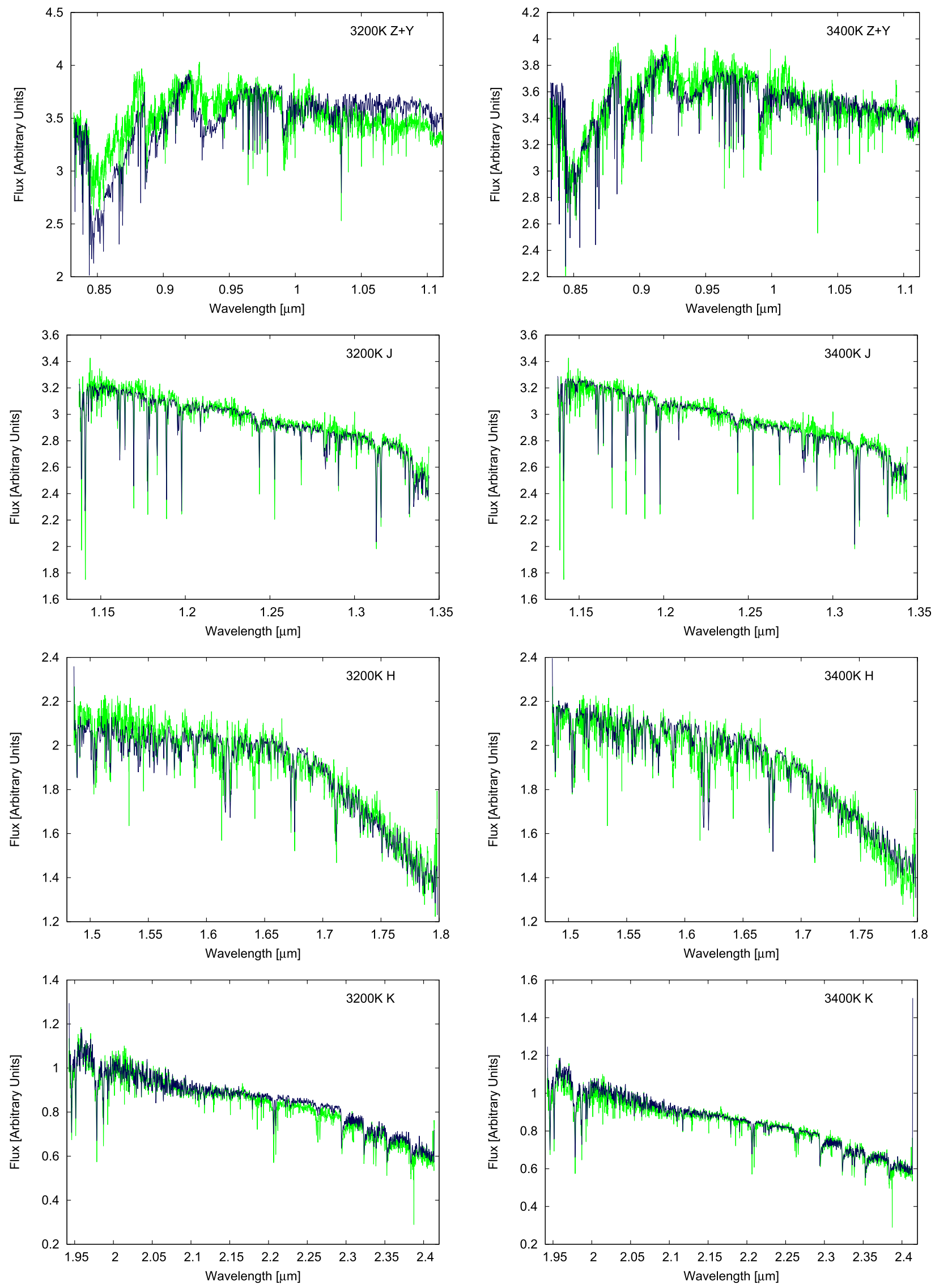

Figure 9. NIR spectra for the primary star of HAT-TR-318-007 from Magellan/FIRE, showing the $(Z+Y), J, H$, and $K$ bands separately (green lines). In the left panel we overlay the BT-Settl template with the highest cross-correlation (blue lines). This template has $T_{\text {eff }}=3200 \mathrm{~K}$ and $[\mathrm{Fe} / \mathrm{H}]=0.3$. In the right panel we overlay a $T_{\text {eff }}=3400 \mathrm{~K},[\mathrm{Fe} / \mathrm{H}]=0.3$ template (blue lines), which provides a better match to the bluest $(Z+Y)$ band, as well as some of the features in the $K$ band. A separate vertical scaling is applied to the templates in each band to provide the best match to the observed spectra. 

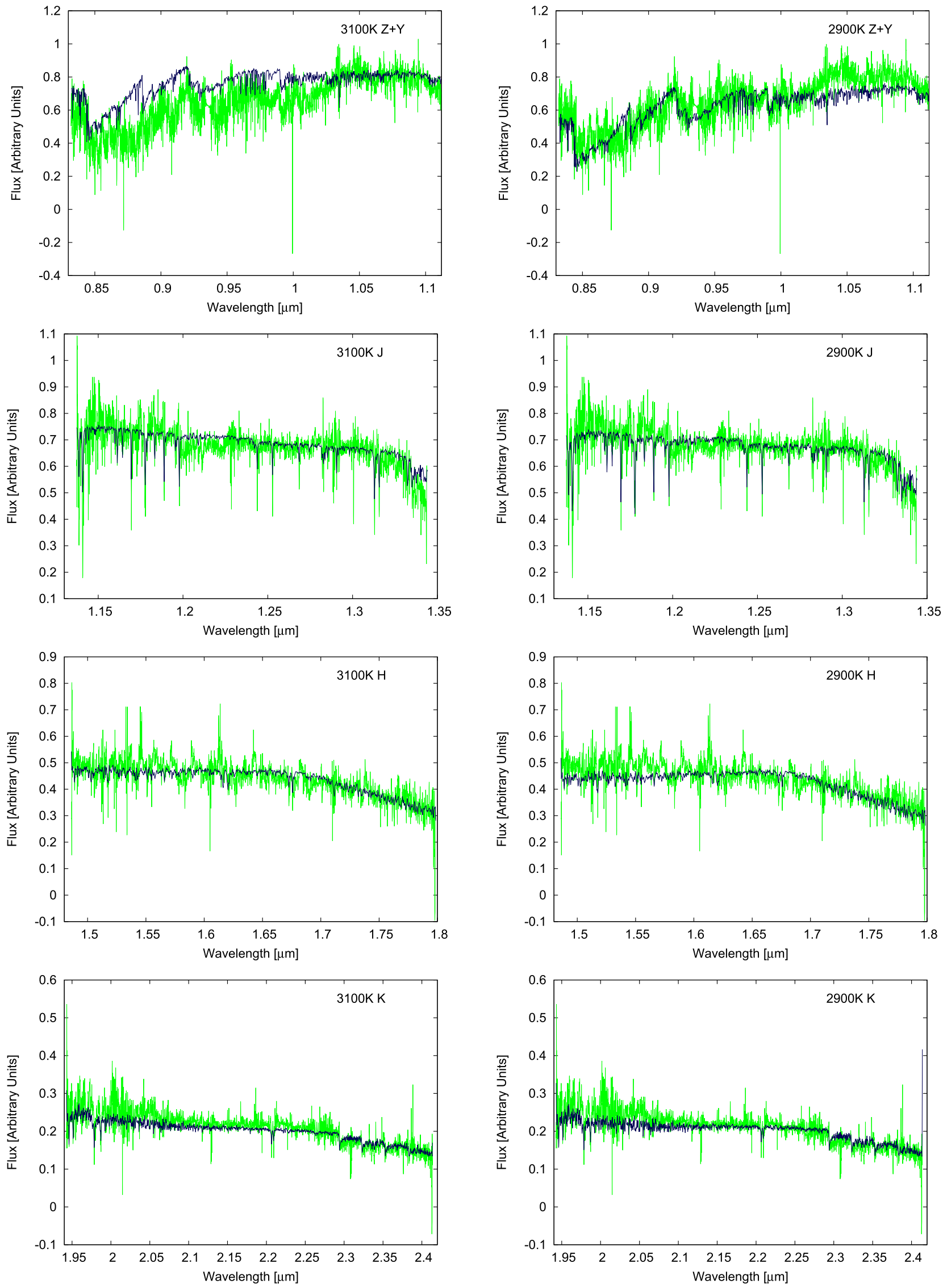

Figure 10. Same as Figure 9, but here we show the secondary component. The overlaid template in the left panel has $T_{\text {eff }}=3100 \mathrm{~K},[\mathrm{Fe} / \mathrm{H}]=0.3$, while in the right panel it has $T_{\text {eff }}=2900 \mathrm{~K},[\mathrm{Fe} / \mathrm{H}]=0.3$. 


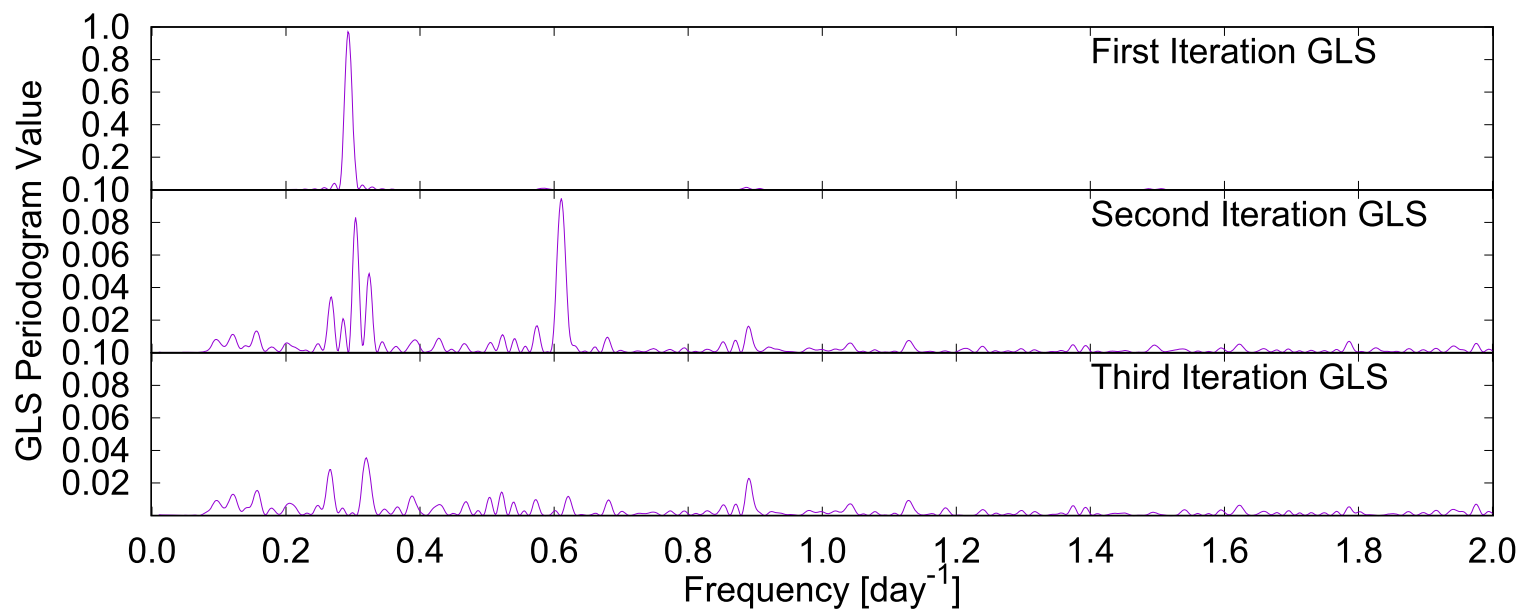

Figure 11. Iterative application of the GLS periodogram to the EVEREST K2 Campaign 5 light curve of HAT-TR-318-007 after removing observations taken during eclipses, removing high flux outliers, and correcting for a systematic variation in the background. The top panel shows the very significant detection of the $P=3.41315_{-0.00032}^{+0.00030}$ day rotational period of the primary star (this value, with its uncertainty, comes from the analysis described in Section 3.2, whereas the actual peak in the GLS periodogram is at $P=3.42005489$ days). The middle panel shows the presence of an additional signal at $P=1.63743194$ days after fitting and subtracting a Fourier series to the light curve with the period fixed to $3.41315_{-0.00032}^{+0.0030}$ days. We adopt double the GLS period in the analysis in Section 3.2, yielding a period of $P=3.28498_{-0.00034}^{+0.00035}$ days for this signal. The bottom panel shows that no additional periodic signals are detected at a significant level after subtracting a pair of Fourier series with periods of $P=3.41315_{-0.00032}^{+0.00030}$ days and $3.28498_{-0.00034}^{+0.00035}$ days. Note the different vertical scales between the three panels. Also note that the full periodogram is calculated up to the Nyquist frequency, but we only display the region of the periodogram where significant peaks are present.
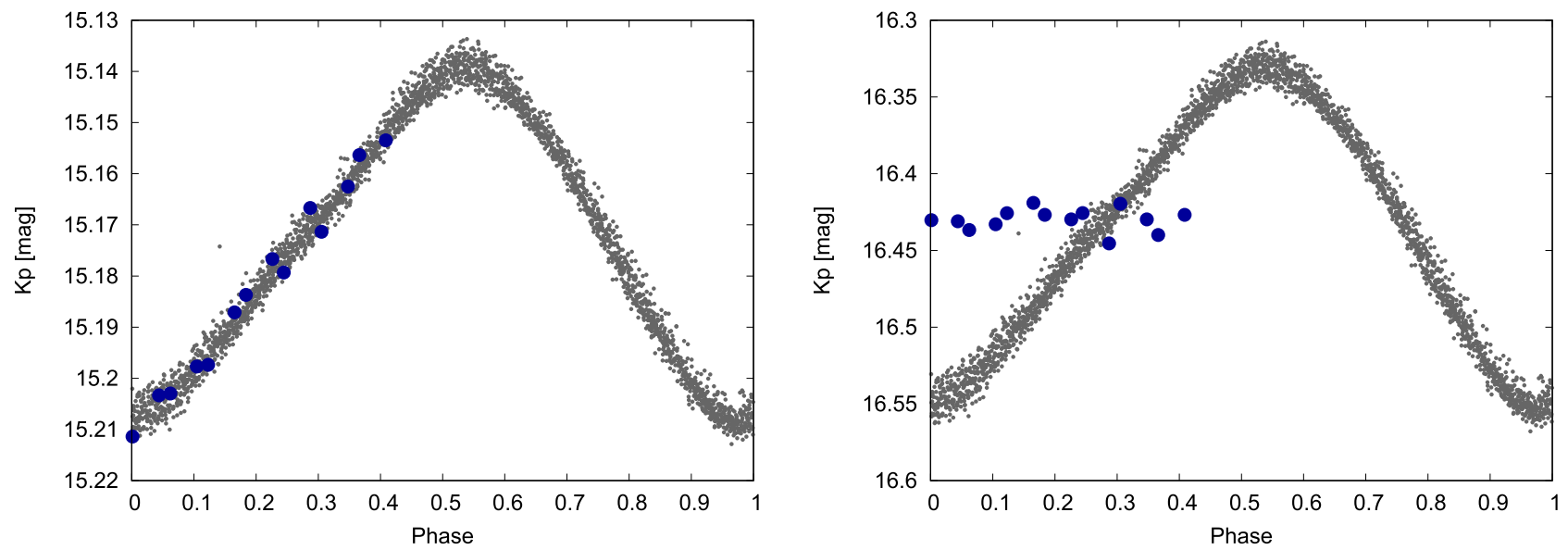

Figure 12. Left: out-of-eclipse $K 2$ observations of HAT-TR-318-007 with a constant flux contribution from the secondary star subtracted and phase-folded at a period of $3.41315_{-0.00032}^{+0.00030}$ days (small gray circles). We also show the brightness of the primary component directly measured from observations obtained during total secondary eclipses (larger blue circles) and phase-folded at the same period. Right: same as the left panel, but here we subtract a constant flux contribution from the primary star (attributing the out-of-eclipse variability to the secondary star), while the larger blue circles show the secondary component brightness measured from the amount of light lost at each secondary eclipse. We conclude that the $P=3.41315_{-0.00032}^{+0.00030}$ day signal originates on the primary star, and we interpret it as the rotation period of that component.

Each KeplerCam and BOS eclipse light curve was treated as an independent time series in the fit, while we also fit each of the four different HATNet light curves and the full $K 2$ light curve independently. To account for the long integration time of $K 2$, we evaluated the full model for the $K 2$ light curve at several points within a 30-minute time bin centered on the BJD time of the observation and averaged these model flux values.

In order to limit the amplitude of the $i$-band out-of-eclipse variability due to spots, we also included a time-binned version of the combined KeplerCam light curve shown in Figure 14, excluding observations taken during the eclipses. Note that this light curve is based on a joint reduction of all of the KeplerCam data and thus contains information about the relative change in brightness between nights, which is not included in the independently reduced KeplerCam light curves that we use to model the eclipses. The joint KeplerCam light curve also includes observations from nights where no eclipse was observed. We perform the time binning to reduce the extent to which we are fitting the same information twice (i.e., the outof-eclipse variations that occur at short timescales). Also note that when we do not include the latter light curve in the fit the model converges to a very large $i$-band spot variability amplitude of $0.14 \mathrm{mag}$, which is inconsistent with the out-ofeclipse observations. Most of the parameters, however, are insensitive to the inclusion of this light curve in the modeling. Exceptions are the $i$-band primary star limb-darkening coefficients and the $i$-band magnitude difference between the primary and secondary stars, which differ by more than $2 \sigma$, and the orbital inclination and $i$-band surface brightness ratio, which differ by more than $1 \sigma$.

We searched our own KeplerCam observations and the Gaia catalog for any resolved neighbors that might contaminate the 

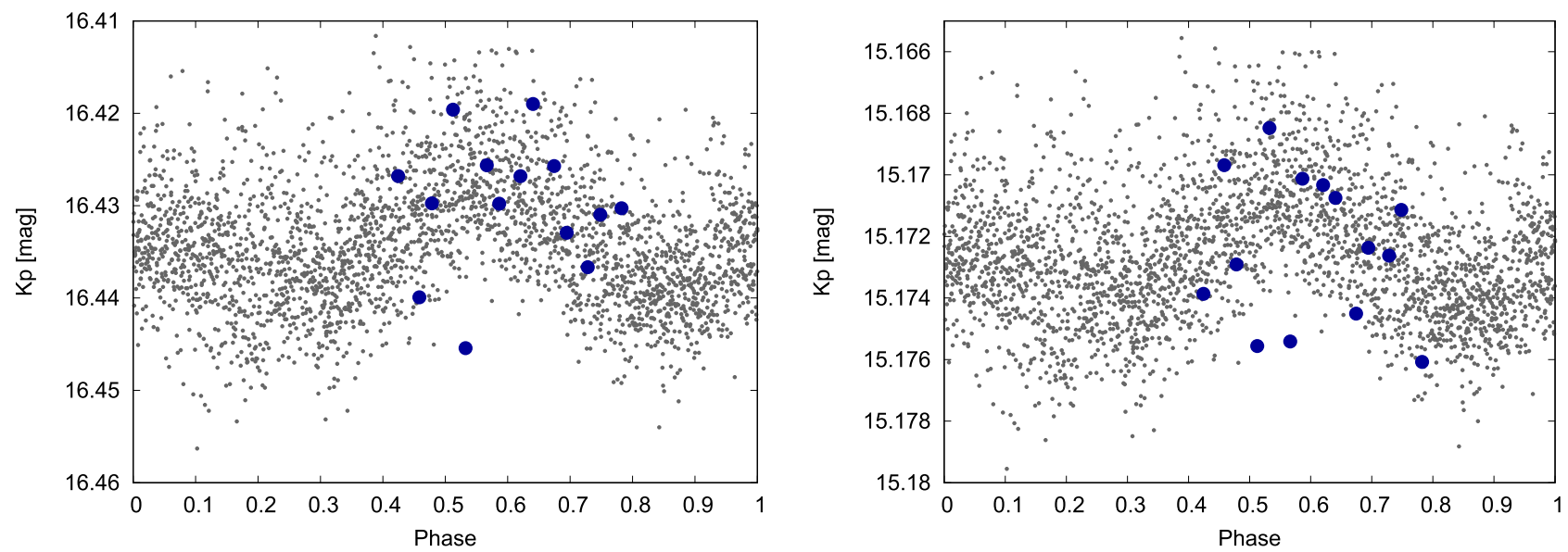

Figure 13. Similar to Figure 12, but here we show observations after subtracting the periodic signal shown in that figure from the flux of the primary star and phasefold the observations at a period of $3.28498_{-0.00034}^{+0.00035}$ days. In this case the left panel shows the result when we attribute the variation to the secondary star, while the right panel shows the result when we attribute it to the primary star. In this case we cannot clearly determine which component of the binary system is giving rise to the observed variation.

photometry, finding no such neighbors down to $\sim 1^{\prime \prime}$ and $G \sim 20$ mag.

Our method for optimizing the parameters in our fit and estimating the uncertainties is similar to that used by Irwin et al. (2011). We used a Differential Evolution Markov Chain (ter Braak 2006; Eastman et al. 2013) to explore the posterior parameter distributions, assuming uniform priors on the adjusted variables. The resulting parameters and $1 \sigma$ uncertainties $(15.85 \%$ to $84.15 \%$ confidence region) that we find for HAT-TR-318-007 are listed in Table 7. Below we discuss a few additional details that are relevant to the modeling of this system.

\subsubsection{Trend Filtering and Error Scaling}

We extended our physical light-curve model with a model for systematic variations due to instrumental or atmospheric effects following the approach of Bakos et al. (2010). The model magnitude for light curve $j$ at time $t_{i}$ is given by

$$
m_{j}\left(t_{i}\right)=M_{\mathrm{phys}, \mathrm{j}}\left(t_{i}\right)+\sum_{k=1}^{N_{\mathrm{EPD}, \mathrm{j}}} a_{j, k} x_{i, j, k}+\sum_{k=1}^{N_{\mathrm{TFA}}} b_{k} y_{i, j, k},
$$

where $M_{\mathrm{phys}, \mathrm{j}}$ is the physical model for light curve $j$, there are $N_{\mathrm{EPD}, \mathrm{j}}$ sets of external parameters to decorrelate (EPD) against for light curve $j$, each of which has value $x_{i, j, k}$, and $N_{\text {TFA }}$ template light curves are used to model additional trends (this is the trend-filtering algorithm, or TFA, due to Kovács et al. 2005). These templates have value $y_{i, j, k}$ at time $i$ for light curve $j$. The parameters $a_{j, k}$ and $b_{k}$ are linear coefficients that are optimized in our fit.

For KeplerCam and BOS, the external parameters that we decorrelate against include the hour angle of the observations (to second order) and three parameters describing the shape of the point-spread function (PSF; each to first order). We use a set of 20 TFA template light curves for the KeplerCam observations and a separate set of five template light curves for the BOS observations.

For $K 2$ we use a set of 12 vectors that define a six-harmonic Fourier series with a period equal to the time span of the data. This accounts for any additional variation in the background or other long-timescale systematics in the EVEREST $K 2$ light curve. While in principle the pixel-level decorrelation of the variations due to the spacecraft roll should be applied simultaneously to the fitting of this large-amplitude variable star, the development of a method to carry out such an analysis is beyond the scope of this paper. We note that the consistency of the eclipsing system model parameters estimated solely from the $K 2$ observations with those estimated solely from the KeplerCam observations indicates that the $K 2$ light curves being analyzed here have not been significantly distorted by the detrending process.

We include the HATNet observations in our modeling as well, but in this case we do not include the EPD or TFA terms. For the HATNet observations we include a dilution factor to account for blending from poorly resolved neighbors and/or effective dilution due to application of TFA before fitting a model.

Because the photometric and RV uncertainties are underestimated, we include scaling factors applied to the errors. These are varied in the fit following the method of Gregory (2005). We note that the most likely values yield a reduced $\chi^{2}$ of 1 , but allowing these to vary rather than adopting the optimal values ensures that the uncertainty in these parameters also contributes to the uncertainties in the other physical parameters.

\subsubsection{Limb Darkening}

For the HATNet $r$-band observations we assume a linear limb-darkening law and fix the coefficients to the values given in Table 7, which are taken from the Claret (2004) tabulations. For the higher-precision $K 2$ and $i$-band observations we fit for the limb-darkening coefficients. We try both a linear limbdarkening law and a square root law. For the square root law we use parameters $c_{1}^{\prime}$ and $c_{2}^{\prime}$, which are related to the traditional parameters $c_{1}$ and $c_{2}$ by

$$
\begin{aligned}
& c_{1}^{\prime}=c_{1}+2 c_{2} / 3 \\
& c_{2}^{\prime}=c_{1}-3 c_{2} / 2
\end{aligned}
$$

for a limb-darkening law of the form

$$
I_{\mu}=I_{0}\left[1-c_{1}(1-\mu)-c_{2}(1-\sqrt{\mu})\right],
$$

with $\mu=\cos (\theta)$, where $\theta$ is the angle between the line of sight and the normal to the stellar surface. This parameterization has the advantage that $c_{1}^{\prime}$ and $c_{2}^{\prime}$ are not strongly correlated, whereas 

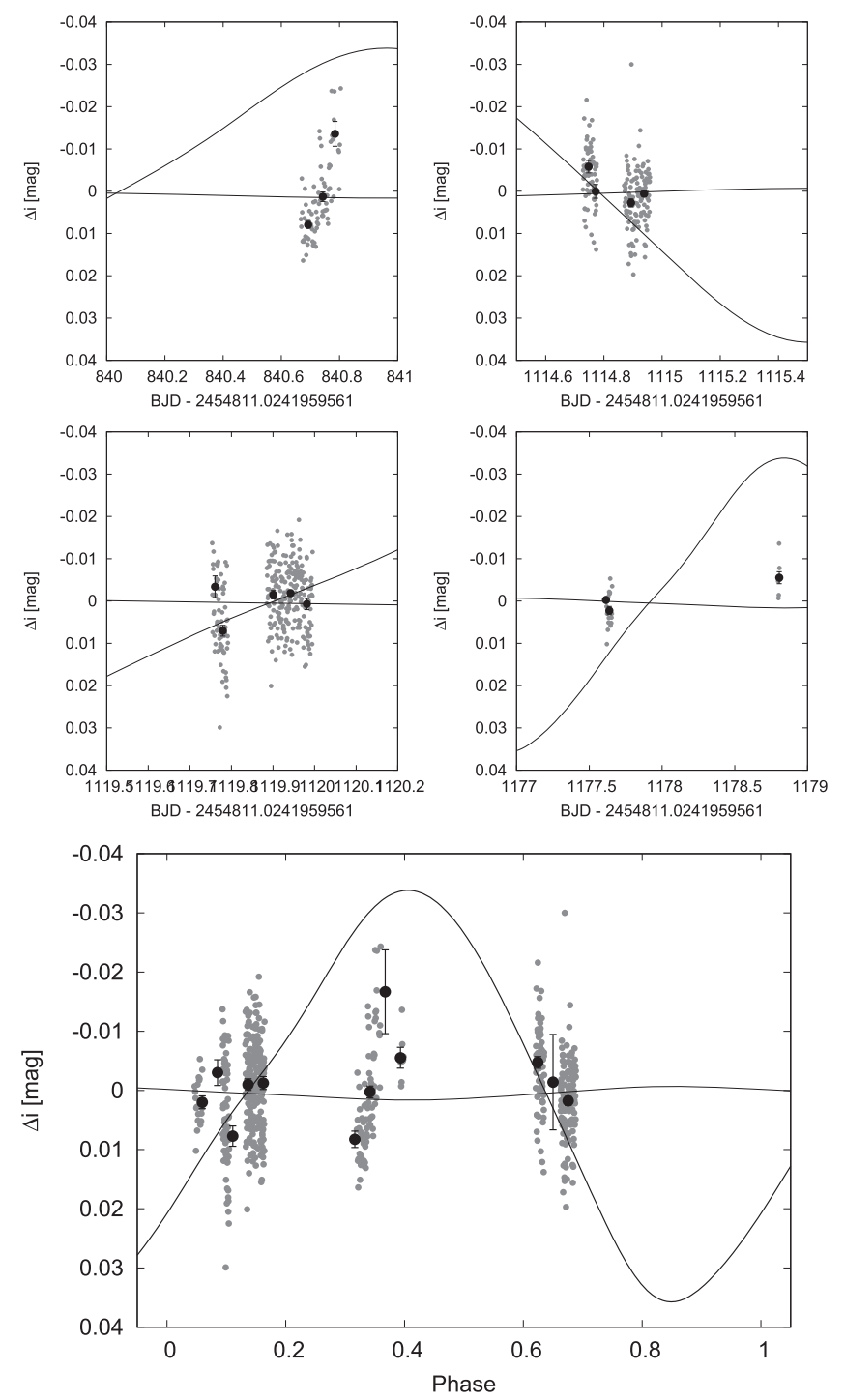

Figure 14. Top: combined KeplerCam $i$-band light curve for HAT-TR-318-007 after removing the primary and secondary eclipses. In order to better see the variability in the time series, we split the figure into four groups of observations that were obtained near in time to each other. The gray filled circles show the individual photometric measurements, while the black filled circles show the measurements binned in time with a bin size of $1.2 \mathrm{hr}$. The large-amplitude line is the best-fit $P=3.41315_{-0.00032}^{+0.00030}$ day rotational signal from the $K 2$ light curve, while the smaller-amplitude line is the same signal with the amplitude fit to the KeplerCam observations. Bottom: same as the top panel, but here we phasefold the observations at the rotational period. Overall the KeplerCam $i$-band observations, which were obtained several years before the $K 2$ data, show significantly smaller amplitude out-of-eclipse variability than was seen in the Kepler bandpass. The variability that is seen in the KeplerCam data does not phase up with the rotational period and may be due to systematic errors in the photometry, which are fit for in modeling the individual KeplerCam eclipse observations, but which are not corrected for in the combined light curve displayed here. We conclude that the rotational variability in the $i$ band has an amplitude $\lesssim 0.01$ mag.

$c_{1}$ and $c_{2}$ are. When fitting the square root law, we find that $c_{1}^{\prime}$ is much more tightly constrained than is $c_{2}^{\prime}$, for both the $K 2$ and ground-based $i$-band observations. We also find that there is no significant difference in $\chi^{2}$ between the two classes of models and that differences in the estimated physical parameters of the binary are unaffected by the choice of limb-darkening law. For this reason our adopted solution is for the linear limb-darkening law, though we list the fitted parameters for the square root law as well. There is no significant difference in the other parameters between the two classes of model.

\subsubsection{Light-travel-time Effect}

There are two light-travel-time effects that are of potential significance to the analysis of this system. These are in addition to the standard barycentric corrections that are applied to the times of observation and RVs to account for the motion of Earth about the center of the solar system. The first effect is due to the orbits of the two components of HAT-TR-318-007 about their own center of mass (see Kaplan 2010; Kaplan et al. 2014). The orbit of the secondary star has a larger semimajor axis about the center of mass than does that of the primary. As a result, primary eclipses are observed to occur slightly earlier, and secondary eclipses slightly later, than would be predicted if the light-travel time were neglected. The observed time difference between primary and secondary eclipses places a strong constraint on $e \cos \omega$, so that neglecting the light-travel time may lead to an incorrect eccentricity measurement. Based on the system parameters determined for HAT-TR-318-007, we expect a correction of $\sim 10 \mathrm{~s}$ to the difference between the times of primary and secondary eclipses due to this effect. Since this is comparable to the precision with which this time difference is constrained based on our observations (our final uncertainty on $T_{c}$ is $\sim 2 \mathrm{~s}$ ), we cannot neglect the effect. We discuss in detail our procedure for accounting for it in Appendix A.

The second light-travel-time effect of potential significance is a small correction to the orbital period due to the recessional velocity of the system. Based on the TRES RVs, we measure a systemic velocity of $30.07 \pm 0.18 \mathrm{~km} \mathrm{~s}^{-1}$, which means that HAT-TR-318-007 recedes from the solar system by 29 lt-s every orbital cycle. Correcting for this effect, the true orbital period of the system is $P=3.34361825 \pm 0.00000020$ days rather than the observed value $P=3.34395390 \pm 0.00000020$ days. This $0.01 \%$ correction to the period results in proportional corrections to the masses, radii, and semimajor axis. As this is approximately two orders of magnitude smaller than the uncertainties on these same parameters, which are currently dominated by uncertainties in the RV semiamplitudes, we do not apply this correction to the parameters listed in Table 7.

\subsubsection{Errors due to Unmodeled Time-correlated Variations}

The light-curve residuals in Figures 2-7 show variations that appear to be correlated in time. These variations may be due to spots, stellar flares, or instrumental artifacts that are not accounted for by the trend-filtering model. In recent years it has become common practice to account for correlated noise using the Gaussian Process Regression method, but an inspection of the moving mean and standard deviation of the light-curve residuals shows that the noise in this case is nonstationary, with sharp variations in the residuals that are likely due to flares and other stellar activity phenomena, and would not be well described with commonly used Gaussian process kernels. In order to estimate the contributions to the parameter uncertainties due to correlated variations that are not accounted for in the model, we carried out a prayer-bead analysis as follows:

1. For each light curve and RV curve we subtract the best-fit model to produce residual light curves and RV curves.

2. For each of the residual light curves we determine the time boundaries for all primary and secondary eclipses observed in that light curve. For each eclipse covered in 
Table 7

System Parameters for HAT-TR-318-007

\begin{tabular}{|c|c|c|c|}
\hline Parameter & Value & Parameter & Value \\
\hline Light-curve Parameters & & Atmospheric Parameters & \\
\hline$T_{c}(\mathrm{BJD})^{\mathrm{b}, \mathrm{c}}$ & $2457214.941784 \pm 0.000027$ & Secondary spectral type & M5.0 \pm 0.7 \\
\hline$b^{\mathrm{d}}$ & $0.0850_{-0.0096}^{+0.0090}$ & $T_{\text {eff, A }} \mathrm{e}^{\mathrm{e}}$ & $3190 \pm 110$ \\
\hline$i(\operatorname{deg})$ & $89.566_{-0.047}^{+0.050}$ & $T_{\text {eff,B }}$ & $3100 \pm 110$ \\
\hline$\left(R_{\mathrm{A}}+R_{\mathrm{B}}\right) / a$ & $0.08844 \pm 0.00022$ & {$[\mathrm{Fe} / \mathrm{H}]_{\mathrm{eff}, \mathrm{B}}$} & $+0.20 \pm 0.11$ \\
\hline$J_{\mathrm{B}} / J_{\mathrm{A}}(r$ band $)$ & $0.590_{-0.058}^{+0.057}$ & & \\
\hline$J_{\mathrm{B}} / J_{\mathrm{A}}(i$ band $)$ & $0.637_{-0.026}^{+0.030}$ & Orbital Parameters & \\
\hline$J_{\mathrm{B}} / J_{\mathrm{A}}(K p$ band $)$ & $0.624 \pm 0.022$ & & \\
\hline Assumed Limb-darkening Coefficients ${ }^{\mathrm{c}}$ & & $\begin{array}{l}K_{\mathrm{A}}\left(\mathrm{km} \mathrm{s}^{-1}\right) \\
K_{\mathrm{B}}\left(\mathrm{km} \mathrm{s}^{-1}\right) \\
\sqrt{e} \cos \omega\end{array}$ & $\begin{array}{c}48.22 \pm 0.17 \\
79.41_{-0.90}^{+0.89} \\
-0.00041_{-0.00016}^{+0.00015}\end{array}$ \\
\hline & & $\gamma\left(\mathrm{km} \mathrm{s}^{-1}\right)^{\mathrm{g}}$ & $30.07 \pm 0.18$ \\
\hline Fitted Limb-darkening Coefficients ${ }^{j}$ & & Physical Parameters & \\
\hline \multicolumn{4}{|l|}{ Linear law } \\
\hline Primary $\mu i$-band & $0.839 \pm 0.035$ & $M_{\mathrm{A}}\left(\mathcal{M}_{\odot}^{\mathrm{N}}\right)$ & $0.448 \pm 0.011$ \\
\hline Secondary $\mu i$-band & $0.16_{-0.12}^{+0.13}$ & $M_{\mathrm{B}}\left(\mathcal{M}_{\odot}^{\mathrm{N}}\right)$ & $0.2721_{-0.0042}^{+0.0041}$ \\
\hline Primary $\mu K p$-band & $0.795_{-0.031}^{+0.032}$ & $R_{\mathrm{A}}\left(\mathcal{R}_{\odot}^{\mathrm{N}}\right)$ & $0.4548_{-0.0036}^{+0.0035}$ \\
\hline Secondary $\mu K p$-band & $0.303_{-0.096}^{+0.095}$ & $R_{\mathrm{B}}\left(\mathcal{R}_{\odot}^{\mathrm{N}}\right)$ & $0.2913_{-0.0024}^{+0.0003}$ \\
\hline Square root law & & $\log g_{\mathrm{A}}(\mathrm{cgs})$ & $4.7740_{-0.0058}^{+0.0056}$ \\
\hline Primary $c_{1}+2 \mathrm{c}_{2} / 3 i$-band & $0.771_{-0.028}^{+0.027}$ & $\log g_{\mathrm{B}}(\mathrm{cgs})$ & $4.9442_{-0.0039}^{+0.0038}$ \\
\hline Primary $c_{1}-3 \mathrm{c}_{2} / 2 i$-band & $0.24_{-0.66}^{+1.19}$ & $\rho_{\mathrm{A}}\left(\mathrm{g} \mathrm{cm}^{-3}\right)$ & $6.720_{-0.072}^{+0.074}$ \\
\hline Secondary $c_{1}+2 \mathrm{c}_{2} / 3 i$-band & $0.198_{-0.108}^{+0.076}$ & $\rho_{\mathrm{B}}\left(\mathrm{g} \mathrm{cm}^{-3}\right)$ & $15.53 \pm 0.22$ \\
\hline
\end{tabular}

Notes. We adopt the nominal solar conversion constants from IAU 2015 Resolution B3 as listed in Prša et al. (2016), using the suggested notation $\mathcal{M}_{\odot}^{\mathrm{N}}$, $\mathcal{R}_{\odot}^{\mathrm{N}}$, and $\mathcal{L}_{\odot}^{\mathrm{N}}$ for these constants. To calculate the bulk density values listed here, we assume the CODATA 2014 value of the gravitational constant (Mohr et al. 2016): $G=(6.67408 \pm 0.00031) \times 10^{-11} \mathrm{~m}^{3} \mathrm{~kg}^{-1} \mathrm{~s}^{-2}$.

${ }^{a}$ Based on the NIR $\mathrm{H}_{2} \mathrm{O}-\mathrm{K}$ spectral index using the calibration by Rojas-Ayala et al. (2012).

$\mathrm{b}$ Times given here, and throughout the paper, are in Barycentric Julian Date (BJD) on the TDB system. Time conversions from UTC to BJD-TDB for the groundbased observations are performed using VARTOOLS (Hartman \& Bakos 2016).

${ }^{c}$ Epoch of primary eclipse.

${ }^{\mathrm{d}}$ Impact parameter during primary eclipse relative to the sum of the radii of the two stars.

${ }^{\mathrm{e}}$ Based on cross-correlating NIR spectra against BT-Settl synthetic templates.

${ }^{\mathrm{f}}$ Based on averaging the $H$ - and $K$-band spectral-index-based metallicities using the Terrien et al. (2012b) calibration.

${ }^{\mathrm{g}} \gamma$ is the systemic radial velocity relative to the solar system barycenter assuming an RV for Barnard's star of $-110.416 \pm 0.180 \mathrm{~km} \mathrm{~s}^{-1}$ from Chubak et al. (2012). This velocity has not been corrected for gravitational redshifts or convective blueshifts. The radial velocity relative to Barnard's star is $\gamma_{\text {rel }}=140.489_{-0.030}^{+0.031} \mathrm{~km} \mathrm{~s}^{-1}$.

${ }^{\mathrm{h}}$ Calculated from the measured stellar radius and spectroscopically determined effective temperature; assumes a solar effective temperature of $T_{\text {eff } \odot \odot}=5772 \mathrm{~K}$.

${ }^{\mathrm{i}}$ Based on the Delfosse et al. (2000) mass $-M_{K}$ relation for M dwarfs, together with the measured masses of the component stars and the 2 MASS $K_{S}$ magnitude of the system; this leads to a lower uncertainty on the distance than using the empirically measured luminosities.

${ }^{\mathrm{j}}$ We fixed the limb-darkening coefficients to linear law values from the Claret (2004) tabulations for the HATNet $r$-band light curves. For the higher-precision followup $i$-band light curves, we allowed the coefficients to vary, trying both a linear and a square root law. Parameters adopted in the table are for the linear law.

the light curve we then choose at random one of the observed time steps $t_{i}$ in that eclipse and set the residual $r_{0}$, and the observational uncertainty $\sigma_{0}$, for the first time step in that eclipse $t_{0}$ equal to the residual $r_{i}$, and observational uncertainty $\sigma_{i}$, from the chosen time step.
We then set $r_{1}=r_{i+1}, \sigma_{1}=\sigma_{i+1}, r_{2}=r_{i+2}, \sigma_{2}=\sigma_{i+2}$, and so on, cycling back to $r_{j}=r_{0}, \sigma_{j}=\sigma_{0}$ when $i+j=N$, with $N$ being the number of points in the eclipse. We perform a similar random shift of the residuals for all of the out-of-eclipse observations in that 
Table 8

Astrometric Measurements of HAT-TR-318-007

\begin{tabular}{|c|c|c|c|c|c|}
\hline $\mathrm{JD}$ & R.A. (deg, J2000) & Decl. (deg, J2000) & R.A. Error (mas) & Decl. Error (mas) & Source \\
\hline $2,433,979$ & 132.637612 & 12.141664 & 180 & 180 & $\overline{\text { USNO A2.0 }}$ \\
\hline $2,450,767.9869$ & 132.637341 & 12.139984 & 61 & 68 & 2MASS \\
\hline $2,453,710.934$ & 132.637279 & 12.139675 & 58 & 50 & SDSS \\
\hline $2,455,651.72551$ & 132.6372576 & 12.1394879 & 29 & 27 & KeplerCam \\
\hline $2,455,925.869055$ & 132.6372521 & 12.1394607 & 18 & 20 & KeplerCam \\
\hline $2,455,930.89952$ & 132.6372534 & 12.1394657 & 14 & 18 & KeplerCam \\
\hline
\end{tabular}

Table 9

Equivalent Widths and Inferred Metallicities from FIRE/Magellan Following Terrien et al. (2012b)

\begin{tabular}{|c|c|c|c|c|c|c|c|c|}
\hline Target & $\mathrm{EW}_{\mathrm{CaH}}$ & $\mathrm{EW}_{\mathrm{KH} 1}$ & $\mathrm{EW}_{\mathrm{NaK}}$ & $\mathrm{EW}_{\mathrm{CaK}}$ & $\mathrm{H}_{2} \mathrm{O}-\mathrm{H}$ & $\mathrm{H}_{2} \mathrm{O}-\mathrm{K}$ & {$[\mathrm{Fe} / \mathrm{H}] \mathrm{H}$} & {$[\mathrm{Fe} / \mathrm{H}]_{\mathrm{K}}$} \\
\hline GJ 205 & $3.47 \pm 0.31$ & $0.727 \pm 0.069$ & $7.61 \pm 0.12$ & $5.59 \pm 0.15$ & $0.910 \pm 0.015$ & $0.877 \pm 0.024$ & $0.39 \pm 0.10$ & $0.498 \pm 0.019$ \\
\hline GJ 250B & $2.45 \pm 0.28$ & $0.481 \pm 0.068$ & $4.80 \pm 0.12$ & $4.06 \pm 0.15$ & $0.944 \pm 0.014$ & $0.979 \pm 0.024$ & $-0.05 \pm 0.10$ & $-0.037 \pm 0.020$ \\
\hline GJ 283 & $0.61 \pm 0.28$ & $1.741 \pm 0.072$ & $4.35 \pm 0.11$ & $1.07 \pm 0.14$ & $0.727 \pm 0.015$ & $0.847 \pm 0.023$ & $-0.25 \pm 0.10$ & $-0.294 \pm 0.020$ \\
\hline GJ 3348B & $1.22 \pm 0.29$ & $0.920 \pm 0.065$ & $5.48 \pm 0.11$ & $3.22 \pm 0.15$ & $0.838 \pm 0.014$ & $0.884 \pm 0.023$ & $-0.33 \pm 0.10$ & $0.018 \pm 0.021$ \\
\hline GJ 352 & $2.27 \pm 0.22$ & $0.738 \pm 0.055$ & $4.71 \pm 0.10$ & $3.61 \pm 0.12$ & $0.875 \pm 0.012$ & $0.888 \pm 0.019$ & $-0.03 \pm 0.08$ & $-0.053 \pm 0.016$ \\
\hline LHS 2065 & $0.56 \pm 0.29$ & $2.881 \pm 0.070$ & $6.80 \pm 0.11$ & $1.05 \pm 0.15$ & $0.762 \pm 0.014$ & $0.797 \pm 0.024$ & $0.20 \pm 0.10$ & $0.046 \pm 0.020$ \\
\hline HAT-TR-318-007A & $2.68 \pm 0.39$ & $1.066 \pm 0.086$ & $7.96 \pm 0.14$ & $5.06 \pm 0.18$ & $0.839 \pm 0.019$ & $0.900 \pm 0.032$ & $0.23 \pm 0.13$ & $0.493 \pm 0.0$ \\
\hline HAT-TR-318-007B ${ }^{\mathrm{b}}$ & $1.41 \pm 0.40$ & $2.545 \pm 0.108$ & $5.91 \pm 0.19$ & $3.08 \pm 0.27$ & $0.914 \pm 0.021$ & $0.871 \pm 0.034$ & $0.43 \pm 0.14$ & $0.068 \pm 0.036$ \\
\hline
\end{tabular}

Notes.

${ }^{a}$ Based on the combined spectrum obtained during total secondary eclipse.

${ }^{\mathrm{b}}$ Based on the disentangled primary and secondary spectra.

light curve. We treat the eclipses and out-of-eclipse observations independently, as we found that systematic variations in the residuals tend to be more pronounced in the eclipses, with differences between the primary and secondary eclipses. While this may introduce an artificial discontinuity in the correlation structure at the edge of the eclipse, treating the eclipses and out-of-eclipse data in a single prayer bead will tend to produce simulated observations with smaller in-eclipse residuals on average than the actual data, leading to underestimated parameter uncertainties.

3. For the primary- and secondary-star RV curves we randomly shift the residuals in time in a similar manner. We treat the two stars independently.

4. We then add the best-fit models that were subtracted from the data back to the shifted residual curves and fit a full model to the simulated data using the downhill simplex algorithm to find the maximum likelihood solution.

We repeat this simulation 1000 times. We then take the standard deviation of the resulting set of parameter values to estimate the systematic uncertainties due to unmodeled systematic variations in the light curves and RV measurements. These errors are then added in quadrature to the $1 \sigma$ uncertainties based on the MCMC analysis to determine the final parameter uncertainties that are listed in Table 7.

\subsection{Atmospheric Parameters}

We use the NIR spectra described in Section 2.4.2 to determine the metallicity and effective temperature of the components of HAT-TR-318-007. We apply two methods to determine these parameters: using empirically calibrated spectral indices, and cross-correlating against theoretical spectral templates. We discuss the results from each method in turn below, with additional details provided in Appendices B and C.

\subsubsection{Spectral Indices}

Rojas-Ayala et al. (2010, 2012, hereafter R10 and R12) and Terrien et al. (2012b, hereafter T12) have determined empirical relations to measure the spectral types and metallicities of M dwarfs using $K$ - and $H$-band spectral indices. We follow these methods, as described in detail in Appendix B, to determine spectral types for the primary and secondary stars of M3.7 \pm 0.7 and M5.0 \pm 0.7 , respectively. These are based on our disentangled spectra. The spectral type determined for the primary star based on the three spectra obtained during total eclipse is M3.6 \pm 0.7 . Using the relation between spectral type and effective temperature given in Bessell (1991), we estimate effective temperatures of the component stars of $T_{\text {eff,A }}=$ $3200 \pm 120 \mathrm{~K}$ and $T_{\text {eff,B }}=3000 \pm 130 \mathrm{~K}$. We also determined separate metallicities for the primary and secondary star of $[\mathrm{Fe} / \mathrm{H}]_{\mathrm{A}}=+0.40 \pm 0.11$ and $[\mathrm{Fe} / \mathrm{H}]_{\mathrm{B}}=+0.20 \pm 0.11$, respectively, which are consistent to within $2 \sigma$. Assuming that both components have the same metallicity, we take the weighted mean of the individual metallicities to estimate a system metallicity of $[\mathrm{Fe} / \mathrm{H}]=+0.298 \pm 0.080$. Systematic uncertainties are included in all of the parameter uncertainties listed here. The equivalent widths and metallicities that we measure following T12 and R12 are listed, for all of our observed stars, in Tables 9 and 10, respectively. 

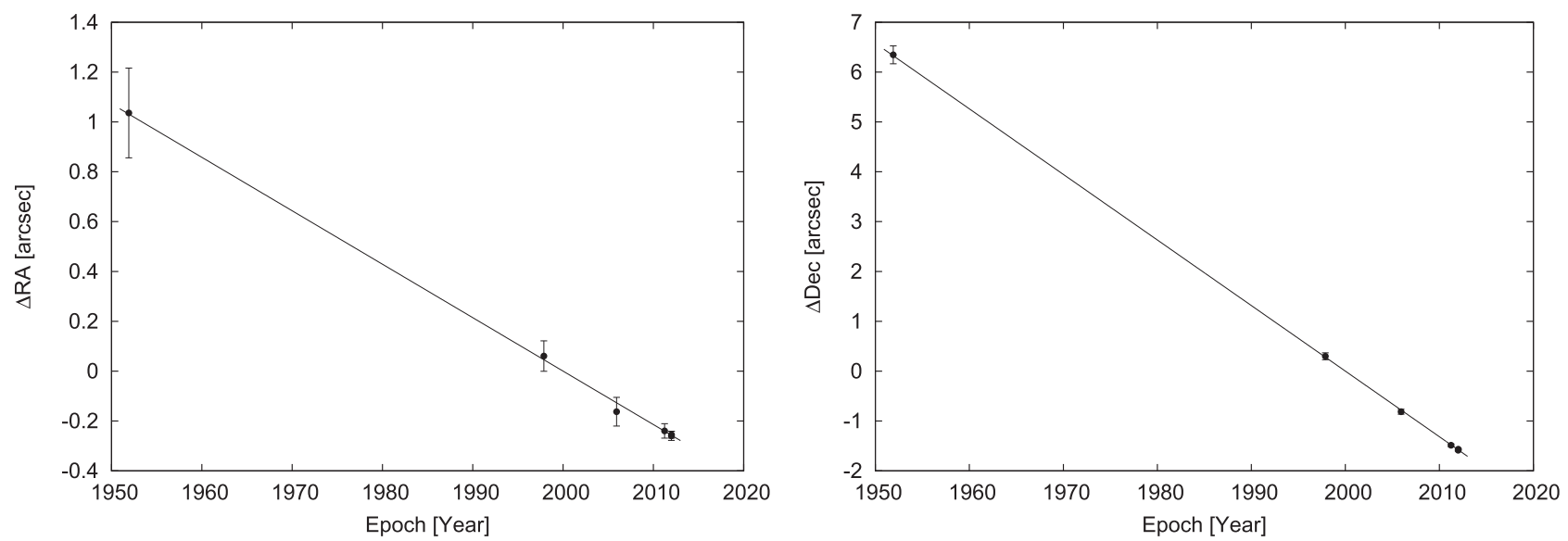

Figure 15. Right ascension (left) and declination (right) vs. time showing the proper motion of HAT-TR-318-007. The data are taken from Table 8 .

Table 10

Equivalent Widths and Inferred Spectral Types and Metallicities from FIRE/Magellan following Rojas-Ayala et al. (2012)

\begin{tabular}{|c|c|c|c|c|c|c|}
\hline Target & $\mathrm{EWNa}_{\mathrm{K}}$ & $\mathrm{EWCa}_{\mathrm{K}}$ & $\mathrm{H}_{2} \mathrm{O}-\mathrm{K}$ & Spec. Type & {$[\mathrm{Fe} / \mathrm{H}]$} & {$[\mathrm{M} / \mathrm{H}]$} \\
\hline GJ 205 & $8.11 \pm 0.43$ & $6.20 \pm 0.25$ & $0.967 \pm 0.010$ & $\mathrm{M} 1.68 \pm 0.24$ & $0.496 \pm 0.060$ & $0.360 \pm 0.043$ \\
\hline GJ 250B & $4.74 \pm 0.43$ & $4.58 \pm 0.26$ & $0.943 \pm 0.010$ & $\mathrm{M} 2.27 \pm 0.23$ & $-0.005 \pm 0.060$ & $-0.004 \pm 0.041$ \\
\hline GJ 283 & $3.64 \pm 0.48$ & $1.10 \pm 0.27$ & $0.737 \pm 0.009$ & $\mathrm{M} 7.16 \pm 0.25$ & $-0.408 \pm 0.073$ & $-0.282 \pm 0.051$ \\
\hline GJ 285 & $7.04 \pm 0.43$ & $4.81 \pm 0.22$ & $0.844 \pm 0.009$ & M4.61 \pm 0.22 & $0.401 \pm 0.060$ & $0.283 \pm 0.041$ \\
\hline GJ 3348B & $5.49 \pm 0.45$ & $3.47 \pm 0.24$ & $0.865 \pm 0.010$ & $\mathrm{M} 4.11 \pm 0.24$ & $0.027 \pm 0.062$ & $0.022 \pm 0.046$ \\
\hline GJ 352 & $4.82 \pm 0.36$ & $3.76 \pm 0.19$ & $0.930 \pm 0.009$ & $\mathrm{M} 2.55 \pm 0.21$ & $-0.080 \pm 0.046$ & $-0.053 \pm 0.035$ \\
\hline LHS 2065 & $6.70 \pm 0.45$ & $1.16 \pm 0.29$ & $0.670 \pm 0.010$ & M8.76 \pm 0.23 & $0.092 \pm 0.072$ & $0.072 \pm 0.053$ \\
\hline NLTT 15867 & $4.14 \pm 0.45$ & $1.84 \pm 0.25$ & $0.841 \pm 0.011$ & M4.72 \pm 0.24 & $-0.328 \pm 0.063$ & $-0.227 \pm 0.041$ \\
\hline HAT-TR-318-007A ${ }^{\mathrm{a}}$ & $8.64 \pm 0.40$ & $5.77 \pm 0.22$ & $0.886 \pm 0.008$ & M3.62 \pm 0.18 & $0.634 \pm 0.054$ & $0.450 \pm 0.037$ \\
\hline HAT-TR-318-007A ${ }^{\mathrm{b}}$ & $8.34 \pm 0.60$ & $5.33 \pm 0.28$ & $0.882 \pm 0.013$ & $\mathrm{M} 3.71 \pm 0.30$ & $0.550 \pm 0.079$ & $0.394 \pm 0.057$ \\
\hline HAT-TR-318-007B ${ }^{\mathrm{b}}$ & $6.76 \pm 0.70$ & $2.53 \pm 0.85$ & $0.828 \pm 0.016$ & $\mathrm{M} 5.01 \pm 0.38$ & $0.077 \pm 0.143$ & $0.062 \pm 0.103$ \\
\hline
\end{tabular}

Notes.

${ }^{\mathrm{a}}$ Based on the combined spectrum obtained during total secondary eclipse.

${ }^{\mathrm{b}}$ Based on the disentangled primary and secondary spectra.

\subsubsection{Cross-correlation against Theoretical Spectral Templates}

As an alternative method to determine the stellar atmospheric parameters, we compare our disentangled NIR spectra to model spectra from the BT-Settl grid (Allard et al. 2011) computed using the Asplund et al. (2009) solar abundances. The details of our method are described in Appendix C. For HAT-TR-318007A and HAT-TR-318-007B we find effective temperatures of $T_{\text {eff, } \mathrm{A}}=3190 \pm 110 \mathrm{~K}$ and $T_{\text {eff,B }}=3100 \pm 110 \mathrm{~K}$, respectively, from cross-correlation, as well as metallicities of $[\mathrm{Fe} / \mathrm{H}]_{\mathrm{A}}=+0.25 \pm 0.13$ and $[\mathrm{Fe} / \mathrm{H}] \mathrm{B}=+0.09 \pm 0.15$, respectively. Combining the metallicities of the primary and secondary components yields a metallicity for the system of $[\mathrm{Fe} / \mathrm{H}]=+0.18 \pm 0.10$, which is consistent with the system metallicity determined from the spectral indices. Table 11 lists the $T_{\text {eff }}$ and $[\mathrm{Fe} / \mathrm{H}]$ values measured through cross-correlation for all of the stars we observed.

The effective temperatures may be combined with the measured stellar radii to determine the stellar luminosities and bolometric magnitudes of $L_{\mathrm{A}}=(1.93 \pm 0.27) \times 10^{-2} \mathcal{L}_{\odot}^{\mathrm{N}}$, $L_{\mathrm{B}}=(7.1 \pm 1.0) \times 10^{-3} \mathcal{L}_{\odot}^{\mathrm{N}}, M_{\mathrm{bol}, \mathrm{A}}=9.03 \pm 0.15 \mathrm{mag}$, and $M_{\mathrm{bol}, \mathrm{B}}=10.12 \pm 0.16 \mathrm{mag}$, where we assume a solar effective temperature of $T_{\mathrm{eff}, \odot}=5772 \mathrm{~K}$ and bolometric magnitude of $M_{\mathrm{bol}, \odot}=4.740 \mathrm{mag}$.

\subsection{Space Velocity}

HAT-TR-318-007 does not have a reliable proper-motion measurement listed in any of the available catalogs. We therefore determine its proper motion using our KeplerCam observations from 2011 and 2012, together with archival measurements given in the USNO A2.0 catalog (circa 1951; Monet 1998), the Two Micron All Sky Survey (2MASS) catalog (1997; Skrutskie et al. 2006), and the Sloan Digital Sky Survey (SDSS) DR7 primary photometric catalog (2005; Abazajian et al. 2009). The epochs and measured positions (in the J2000 equinox) are collected in Table 8 . Figure 15 shows the change in R.A. and decl. over time, together with our best-fit model. From these observations we measure a proper motion of $\mu_{\text {R.A. }}=$ $-21.0 \pm 2.4 \mathrm{mas} \mathrm{yr}^{-1}$ and $\mu_{\text {decl. }}=-131.6 \pm 2.5 \mathrm{mas} \mathrm{yr}^{-1}$, where we follow the convention that the change in the R.A. coordinate is given by $\mu_{\text {R.A. }} / \cos ($ decl.). We also determine a reference position of R.A. $=08^{\mathrm{h}} 50^{\mathrm{m}} 32^{\mathrm{s}} .9578$, decl. $=$ $+12^{\circ} 08^{\prime} 23$ ". 644 (Equinox J2000, Epoch J2000). We note that the observational precision is insufficient to determine the parallax. When we try to fit for the parallax, we find $\pi=-2 \pm 20$ mas, whereas the expected value is $\pi=8.09 \pm 0.25$ mas. We therefore fix the parallax to zero in fitting for the proper motion. We expect the parallax of this system to be determined in the next year with the release of Gaia DR2. 
Table 11

Atmospheric Parameters from FIRE/Magellan Based on Cross-correlation with BT-Settl Synthetic Templates

\begin{tabular}{lcr}
\hline \hline \multicolumn{1}{c}{ Target } & $\begin{array}{c}T_{\text {eff }} \\
(\mathrm{K})\end{array}$ & \multicolumn{1}{c}{$[\mathrm{Fe} / \mathrm{H}]$} \\
\hline GJ 205 & $3870 \pm 100$ & $0.24 \pm 0.15$ \\
GJ 250B & $3600 \pm 100$ & $-0.13 \pm 0.15$ \\
GJ 283 & $2690 \pm 100$ & $-0.31 \pm 0.14$ \\
GJ 285 & $2050 \pm 110$ & $0.27 \pm 0.14$ \\
GJ 3348B & $3160 \pm 100$ & $-0.05 \pm 0.15$ \\
GJ 352 & $3480 \pm 100$ & $-0.03 \pm 0.15$ \\
LHS 2065 & $2490 \pm 110$ & $0.03 \pm 0.15$ \\
NLTT 15867 & $2980 \pm 110$ & $0.09 \pm 0.15$ \\
HAT-TR-318-007A ${ }^{\mathrm{a}}$ & $3200 \pm 100$ & $0.23 \pm 0.15$ \\
HAT-TR-318-007A & $3190 \pm 110$ & $0.25 \pm 0.13$ \\
HAT-TR-318-007B & & $0.09 \pm 0.15$ \\
\hline
\end{tabular}

${ }^{a}$ The spectrum of HAT-TR-318-007A obtained during total secondary eclipse.

${ }^{b}$ Disentangled spectra of HAT-TR-318-007A and HAT-TR-318-007B.

We use the measured proper motion, together with the $\gamma \mathrm{RV}$ and distance from our best-fit model, to determine the $U V W$ space motion of HAT-TR-318-007 following Johnson \& Soderblom (1987). We find $U=-1.9 \pm$ $1.2 \mathrm{~km} \mathrm{~s}^{-1}, \quad V=-81.0 \pm 2.4 \mathrm{~km} \mathrm{~s}^{-1}, \quad$ and $W=-20.9 \pm$ $1.7 \mathrm{~km} \mathrm{~s}^{-1}$, with the convention that $U$ increases toward the Galactic center. Based on the SDSS DR7 kinematic model for the Galaxy (Bond et al. 2010), 99.5\% of stars with the Galactic position and velocity of HAT-TR-318-007 are members of the disk. The classification of HAT-TR-318-007 as a member of the Galactic disk is consistent with the high metallicity measured for the system.

\section{Discussion}

\subsection{Significance of Total Eclipses}

The presence of total eclipses in this system provides multiple benefits. In addition to facilitating the disentanglement of the spectra for both component stars (Section 2.4.2), it also allows many of the light-curve parameters to be measured with significantly better precision than would be possible for a grazing system. Although a thorough demonstration of this is beyond the scope of this paper, we carried out a few tests based on a preliminary analysis that considered only the ground-based observations (this work was done prior to the $K 2$ observations). We injected simulated grazing eclipses into the residual light curves from our best-fit model. For the grazing model we adopted the parameters for our best-fit model of HAT-TR-318-007, except we set the impact parameter to $b=0.3$. We then used the DEMC method to fit a model to the simulated data and to determine the resulting parameter uncertainties. We found that for the simulated grazing system $R_{\mathrm{B}} / R_{\mathrm{A}},\left(R_{\mathrm{A}}+R_{\mathrm{B}}\right) / a$, and $i$ had statistical uncertainties that were $4.7,3.6$, and 2.1 times larger than the statistical uncertainties for these same parameters when fitting the actual system with total eclipses. We also found that the limbdarkening coefficients for the primary star could be determined to much better precision when total eclipses are present. Assuming a linear limb-darkening law, the statistical uncertainties on the limbdarkening coefficient for the primary and secondary stars were 3.6 and 1.2 times higher for the grazing system than for the total eclipsing system. The only parameter that we found would be determined with better precision in the grazing system is $J_{\mathrm{B}} / J_{\mathrm{A}}$ in the $i$ band, which has a precision for the grazing system that is 0.90 times that of the precision for the total eclipsing system.

While including the $K 2$ observations and accounting for the starspot modulation would affect the relative uncertainties from a grazing versus total eclipsing system, we still expect that the total eclipsing system would generally have lower uncertainties than the grazing system.

\subsection{Asynchronous Rotation}

We find that the photometric rotation period of the primary star, $P_{\text {rot, } \mathrm{A}}=3.41315_{-0.00032}^{+0.00030}$ days, is slightly longer than the orbital period of the system, $P_{\text {orb }}=3.34395390 \pm 0.00000020$ days. A similar slight difference from synchronous rotation was also observed for at least one of the $\mathrm{M}$ dwarf components in the binary LP 661-13 discovered by Dittmann et al. (2017). More generally, Balaji et al. (2015) analyzed a sample of 414 short-period, nearcontact and contact binaries observed by Kepler and found that at least $50 \%$ of these systems exhibit starspot rotation that is not exactly synchronized with the orbital periods. One possible explanation for the close, but not exact, synchronization is differential rotation. As shown by Scharlemann (1982), the tidal forces that lead to synchronization are insufficient to suppress differential rotation within the star. Stars will have a co-rotating latitude, with rotation that is slower than the orbital period above that latitude and rotation that is faster than the orbital period below that latitude. The observations by Balaji et al. (2015) appear to be consistent with this picture. They find that the differences between the observed spin periods of close binary components and the orbital periods are consistent with being the result of modest differential rotation on these stars, with a differential rotation parameter that is lower on average by a factor of $\sim 3$ compared to what is found for isolated stars. For our observations of HAT-TR$318-007 \mathrm{~A}$, the relative difference in frequency between the rotation and orbit $\alpha=\left(\Omega_{\text {orb }}-\Omega_{\text {rot }}\right) / \Omega_{\text {orb }}=0.02$ is well within the range of $\alpha=\left(\Omega_{\mathrm{eq}}-\Omega_{\text {pole }}\right) / \Omega_{\mathrm{eq}}$ found for isolated Kepler stars by Reinhold et al. (2013).

In addition to differential rotation, another potential source of asynchronous rotation is a magnetized wind carrying angular momentum away from the system. As discussed by Keppens (1997), magnetized winds will cause main-sequence stars in close binaries to have rotation periods that are slightly slower than the orbital period. In effect, the wind applies a torque that acts to spin down the star and may balance the tidal torque, which decreases as the system approaches synchronization. The amplitude of this effect depends on the wind strength, magnetic field strength, their dependence on rotation period, and the tidal dissipation rate, none of which are well determined for M dwarf stars.

Interestingly, the orbital period of the system is very close to the mean of the two additional periods $P=3.41315_{-0.00032}^{+0.00030}$ days and $P=3.28498_{-0.00034}^{+0.00035}$ days identified in the $K 2$ light curve. As shown in Section 3.1, we cannot determine unambiguously whether the shorter-period signal $P=3.28498_{-0.00034}^{+0.00035}$ days arises on the primary or secondary star. If it arises on the primary star, then the existence of two rotation signals from this star with periods spanning the orbital period would be consistent with the differential rotation scenario, where the star has two active latitudes above and below the co-rotation latitude. 


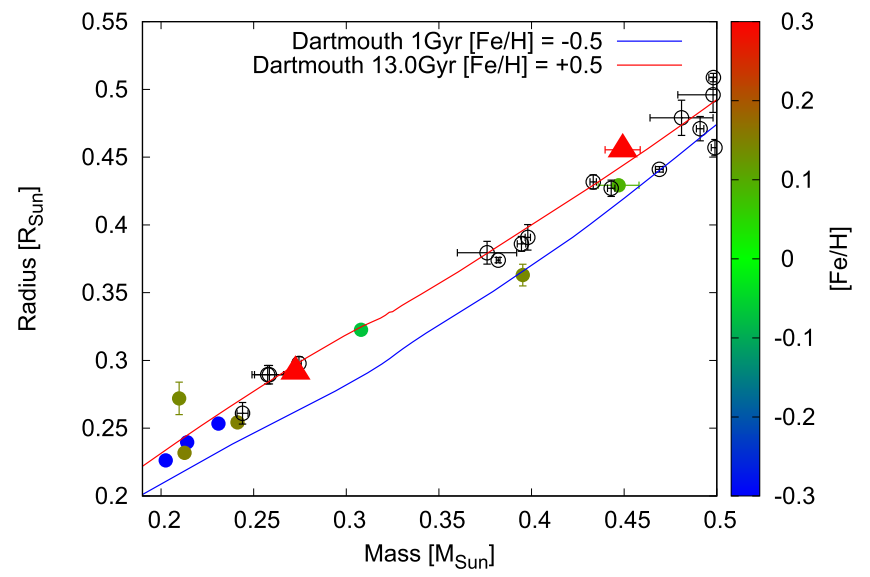

Figure 16. Mass-radius diagram for $\mathrm{M}$ dwarfs with $0.2 \mathcal{M}_{\odot}^{\mathrm{N}}<M<0.5 \mathcal{M}_{\odot}^{\mathrm{N}}$ and masses and radii determined to better than $5 \%$ precision. The color scale of the points indicates the metallicity, if known. Large filled triangles show the components of HAT-TR-318-007; smaller circles show other M dwarfs with parameters given in Table 12. Black open circles are used for systems without a measured metallicity. The lines show theoretical mass-radius relations from the Dartmouth (Dotter et al. 2008) models. Most objects fall between the $1 \mathrm{Gyr}$, $[\mathrm{Fe} / \mathrm{H}]=-0.5$ and the $13 \mathrm{Gyr},[\mathrm{Fe} / \mathrm{H}]=+0.5$ Dartmouth models, indicating that these models can explain the observations if the stars are old and/or metalrich.

\subsection{Comparison with Other M Dwarf Systems and Theoretical Models}

There are 22 other $\mathrm{M}$ dwarf stars in eclipsing binaries with masses between 0.2 and $0.5 \mathcal{M}_{\odot}^{\mathrm{N}}$, and with masses and radii measured to better than $5 \%$ precision. These are collected in Table 12. Although Ramón Iglesias-Marzoa et al. (2017) report parameters for the star T-Cyg-12664B that pass these cuts, we exclude it from this table, as Han et al. (2017), who make use of RVs measured for both the primary and secondary stars, find higher uncertainty values that differ substantially from those of Ramón Iglesias-Marzoa et al. (2017). Figures 16-18 show the mass-radius, mass- $T_{\text {eff }}$, and mass-luminosity diagrams for these objects, respectively. Overplotted on these are isochrones from the Dartmouth theoretical stellar evolution models (Dotter et al. 2008). While the observed radii are generally above the $[\mathrm{Fe} / \mathrm{H}]=0$, age $=1 \mathrm{Gyr}$ isochrone, they are mostly below, or consistent with, the $[\mathrm{Fe} / \mathrm{H}]=+0.5$, age $=13 \mathrm{Gyr}$ isochrone. This indicates that most objects can be explained by these models if they are old and/or metal-rich. While the masses and radii may be consistent with these models, the observed effective temperatures are systematically lower than the models. The luminosities, which are computed from the effective temperatures and radii, are also lower than the models.

For systems with an observed metallicity we can provide a more quantitative comparison to the models as follows. To compare the model to the observed parameters for a system (e.g., the masses, radii, and metallicity), we make use of the following likelihood function:

$$
L \propto \exp -\frac{1}{2}(\boldsymbol{X}-\boldsymbol{X} \boldsymbol{O})^{\prime} \boldsymbol{A}^{-1}(\boldsymbol{X}-\boldsymbol{X} \boldsymbol{0}),
$$

where $\boldsymbol{X}$ is a vector consisting of the observations (e.g., two masses, two radii, and the system metallicity), $\boldsymbol{X} \boldsymbol{O}$ is a vector of the mean values for these parameters, and $\boldsymbol{A}$ is the covariance matrix between these parameters (for systems taken from the literature we assume that none of the parameters are correlated). For HAT-TR318-007 we determine this from the MCMC chains produced in

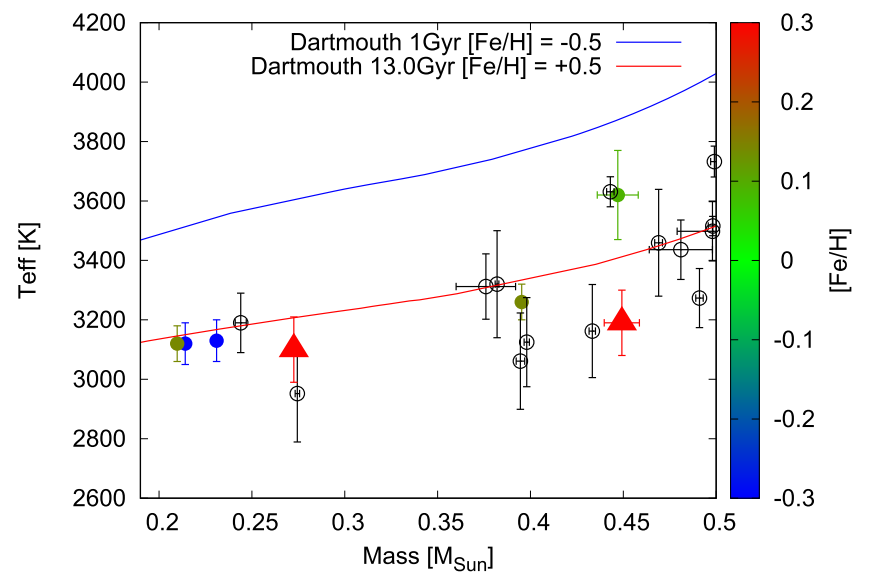

Figure 17. Similar to Figure 16, but here we show the mass- $T_{\text {eff }}$ diagram. The observed temperatures are systematically below the theoretical models, with even the $13 \mathrm{Gyr},[\mathrm{Fe} / \mathrm{H}]=+0.5$ model being above most of the observations.

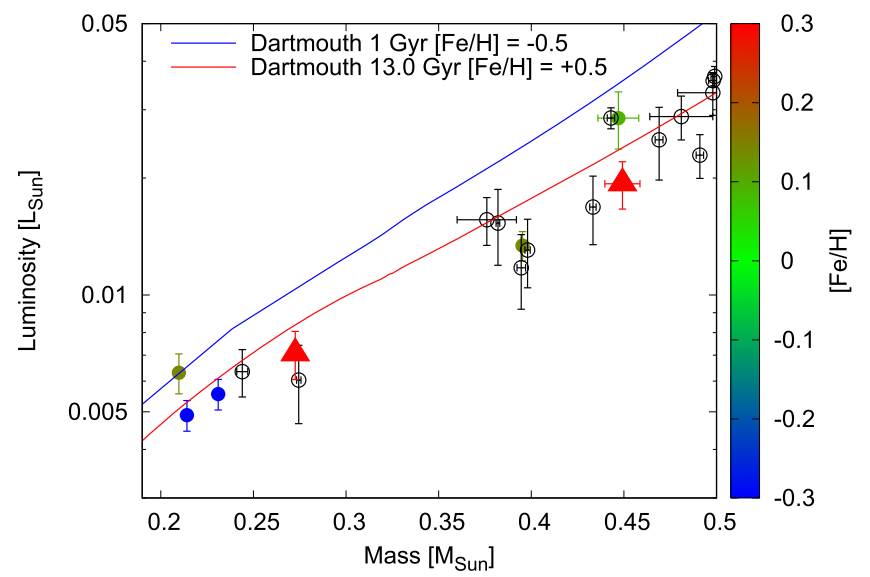

Figure 18. Similar to Figure 16, but here we show the mass-luminosity diagram. The observed luminosities are determined from the measured radii and temperatures and are systematically below the theoretical models, with even the $13 \mathrm{Gyr},[\mathrm{Fe} / \mathrm{H}]=+0.5$ model being above most of the observations.

fitting the light curves and RV data; for other objects we assume uncorrelated errors between the parameters and use the $1 \sigma$ errors presented in their papers. To fit the model to the data, we interpolate within a precomputed grid of isochrones to determine the radii for each trial set of parameters. The grid is calculated using the Dartmouth web interface ${ }^{12}$ assuming $[\alpha / \mathrm{Fe}]=0$ for $[\mathrm{Fe} / \mathrm{H}] \geqslant 0$ and $[\alpha / \mathrm{Fe}]=+0.2$ for $[\mathrm{Fe} / \mathrm{H}]<0$. We restrict the parameter search to $-2.0 \leqslant[\mathrm{Fe} / \mathrm{H}] \leqslant+0.5$ and $1 \mathrm{Gyr}<$ age $<13.8 \mathrm{Gyr}$, where the upper limit on the age is taken to be the age of the universe based on Planck results (Planck Collaboration et al. 2014). We carry out a DEMC analysis to determine the parameters and their uncertainties.

Note that here we often treat directly observed parameters, such as the metallicity or mass of each star, as free parameters in the model that are optimized in the process. Doing this accounts for the uncertainties in the observed values and the contribution of these uncertainties to the values predicted by the model for other parameters, such as the stellar radii or effective temperatures.

We applied this analysis to HAT-TR-318-007 and to each of the six systems in Table 12 with measured metallicities. For

\footnotetext{
12 http://stellar.dartmouth.edu/models/grid.html
} 
Table 12

Literature M Dwarfs in Eclipsing Binary Systems with Masses between $0.2 \mathcal{M}_{\odot}^{\mathrm{N}}<M<0.5 \mathcal{M}_{\odot}^{\mathrm{N}}$, and with Masses and Radii Determined to Better than $5 \%$ Precision

\begin{tabular}{|c|c|c|c|c|c|}
\hline Star & $\begin{array}{l}\text { Mass } \\
\left(\mathcal{M}_{\odot}^{\mathrm{N}}\right)\end{array}$ & $\begin{array}{c}\text { Radius } \\
\left(\mathcal{R}_{\odot}^{\mathrm{N}}\right)\end{array}$ & $\begin{array}{l}T_{\text {eff }} \\
(\mathrm{K})\end{array}$ & {$[\mathrm{Fe} / \mathrm{H}]$} & Reference(s) \\
\hline MG1-646680A & $0.499 \pm 0.002$ & $0.457 \pm 0.006$ & $3730 \pm 50$ & $\ldots$ & (Kraus et al. 2011) \\
\hline NSVS 01031772B & $0.4982 \pm 0.0025$ & $0.5087 \pm 0.0031$ & $3520 \pm 30$ & $\ldots$ & (Lopez-Morales et al. 2006) \\
\hline WTS 19b-2-01387A & $0.498 \pm 0.019$ & $0.496 \pm 0.013$ & $3498 \pm 100$ & $\ldots$ & (Birkby et al. 2012) \\
\hline MG1-78457B & $0.491 \pm 0.002$ & $0.471 \pm 0.009$ & $3270 \pm 100$ & $\ldots$ & (Kraus et al. 2011) \\
\hline WTS 19b-2-01387B & $0.481 \pm 0.017$ & $0.479 \pm 0.013$ & $3436 \pm 100$ & $\cdots$ & (Birkby et al. 2012) \\
\hline MG1-2056316A & $0.4690 \pm 0.0021$ & $0.441 \pm 0.002$ & $3460 \pm 180$ & $\ldots$ & (Kraus et al. 2011) \\
\hline WOCS $23009 \mathrm{~B}^{\mathrm{a}}$ & $0.447 \pm 0.011$ & $0.4292 \pm 0.0033$ & $3620 \pm 150$ & $+0.09 \pm 0.03$ & (Sandquist et al. 2013) \\
\hline MG1-646680B & $0.443 \pm 0.002$ & $0.427 \pm 0.006$ & $3630 \pm 50$ & $\ldots$ & (Kraus et al. 2011) \\
\hline CU Cnc A & $0.4333 \pm 0.0017$ & $0.4317 \pm 0.0052$ & $3160 \pm 150$ & $\ldots$ & (Ribas 2003) \\
\hline CU Cnc B & $0.3980 \pm 0.0014$ & $0.3908 \pm 0.0094$ & $3130 \pm 150$ & $\ldots$ & (Ribas 2003) \\
\hline PTFEB132.707+19.810 $\mathrm{A}^{\mathrm{b}}$ & $0.3953 \pm 0.0020$ & $0.363 \pm 0.008$ & $3260 \pm 60$ & $+0.14 \pm 0.04$ & (Kraus et al. 2017) \\
\hline LSPM J1112+7626A & $0.3946 \pm 0.0023$ & $0.3860 \pm 0.0055$ & $3060 \pm 160$ & $\ldots$ & (Irwin et al. 2011) \\
\hline MG1-2056316B & $0.382 \pm 0.001$ & $0.374 \pm 0.002$ & $3320 \pm 180$ & $\ldots$ & (Kraus et al. 2011) \\
\hline GJ 3236A & $0.376 \pm 0.016$ & $0.3795 \pm 0.0084$ & $3310 \pm 110$ & $\ldots$ & (Irwin et al. 2009) \\
\hline LP $661-13 A^{c}$ & $0.30795 \pm 0.00084$ & $0.3226 \pm 0.0033$ & $\ldots$ & $-0.07 \pm 0.1$ & (Dittmann et al. 2017) \\
\hline LSPM J1112+7626B & $0.2745 \pm 0.0012$ & $0.2978 \pm 0.0049$ & $2950 \pm 160$ & $\ldots$ & (Irwin et al. 2011) \\
\hline 1RXS J154727.5+450803B & $0.2585 \pm 0.0080$ & $0.2895 \pm 0.0068$ & $\ldots$ & $\ldots$ & (Hartman et al. 2011) \\
\hline 1RXS J154727.5+450803A & $0.2576 \pm 0.0085$ & $0.2895 \pm 0.0068$ & $\ldots$ & $\ldots$ & (Hartman et al. 2011) \\
\hline HATS551-027A & $0.244 \pm 0.003$ & $0.261_{-0.009}^{+0.006}$ & $3190 \pm 100$ & $\ldots$ & (Zhou et al. 2015) \\
\hline KOI-126B ${ }^{\mathrm{d}}$ & $0.2413 \pm 0.0030$ & $0.2543 \pm 0.0014$ & $\ldots$ & $+0.15 \pm 0.08$ & (Carter et al. 2011) \\
\hline $\mathrm{CM}$ Dra $\mathrm{A}^{\mathrm{e}}$ & $0.2310 \pm 0.0009$ & $0.2534 \pm 0.0019$ & $3130 \pm 70$ & $-0.30 \pm 0.12$ & (Morales et al. 2009; Terrien et al. 2012b) \\
\hline $\mathrm{CM}$ Dra $\mathrm{B}^{\mathrm{e}}$ & $0.2141 \pm 0.0010$ & $0.2396 \pm 0.0015$ & $3120 \pm 70$ & $-0.30 \pm 0.12$ & (Morales et al. 2009; Terrien et al. 2012b) \\
\hline KOI-126C ${ }^{d}$ & $0.2127 \pm 0.0026$ & $0.2318 \pm 0.0013$ & $\ldots$ & $+0.15 \pm 0.08$ & (Carter et al. 2011) \\
\hline PTFEB132.707+19.810B ${ }^{b}$ & $0.2098 \pm 0.0014$ & $0.272 \pm 0.012$ & $3120 \pm 60$ & $+0.14 \pm 0.04$ & (Kraus et al. 2017) \\
\hline Kepler-16B ${ }^{\mathrm{f}}$ & $0.20255 \pm 0.00066$ & $0.22623 \pm 0.00059$ & $\ldots$ & $-0.3 \pm 0.2$ & (Doyle et al. 2011) \\
\hline
\end{tabular}

Notes. Except where noted, stars are components of double-lined eclipsing binary systems. We exclude stars with white dwarf binary companions; such systems may have undergone significant mass transfer.

${ }^{\mathrm{a}}$ WOCS $23009 \mathrm{~B}$ is the secondary component of a single-lined binary system with an $M=1.468 \pm 0.030 \mathcal{M}_{\odot}^{\mathrm{N}}$ evolved primary star. This binary system is a member of the open cluster NGC 6819 . The listed $[\mathrm{Fe} / \mathrm{H}]$ is the value for the cluster.

${ }^{b}$ PTFEB132.707+19.810 is a member of the Praesepe open cluster, and the adopted metallicity is the value for the cluster. Note that Gillen et al. (2017) independently identified this as a binary, which they label AD 3814. They measure masses of $0.3813 \pm 0.0074 M_{\odot}$ and $0.2022 \pm 0.0045 M_{\odot}$ and radii of $0.3610 \pm 0.0033 R_{\odot}$ and $0.2256_{-0.0049}^{+0.0063} R_{\odot}$ for the primary and secondary stars, respectively.

${ }^{\mathrm{c}}$ The metallicity of the LP 661-13 eclipsing binary system was not determined spectroscopically, but was estimated using the absolute $K_{s}$ magnitude and the MEarth $-K_{S}$ broadband color following Dittmann et al. (2016).

${ }^{\mathrm{d}}$ KOI-126B and KOI-126C are components of a triply eclipsing hierarchical triple system. The primary star has a mass of $M=1.347 \pm 0.032 \mathcal{M}_{\odot}^{\mathrm{N}}$. Only light from the primary star has been detected in the spectrum. The listed $[\mathrm{Fe} / \mathrm{H}]$ is the value determined spectroscopically for the primary. The triple eclipses, together with the RVs for the primary star, enable a determination of the masses and radii of both stars that is independent of stellar evolution models.

${ }^{\mathrm{e}}$ Feiden \& Chaboyer (2014) argue that $\mathrm{CM}$ Dra has $[\mathrm{Fe} / \mathrm{H}] \sim 0$ dex and $[\alpha / \mathrm{Fe}] \gtrsim+0.2 \mathrm{dex}$.

${ }^{\mathrm{f}}$ Kepler-16B is the secondary component of a binary system with an $M=0.6897 \pm 0.0035 \mathcal{M}_{\odot}^{\mathrm{N}}$ primary star. Light from the secondary star has not been detected within the spectrum; however, there is a transiting circumbinary planet whose transits around each stellar component, in conjunction with the observed RVs for the primary star, allow a determination of the masses and radii of both stars that is independent of stellar evolution models. The listed $[\mathrm{Fe} / \mathrm{H}]$ is the $[\mathrm{M} / \mathrm{H}] \mathrm{value}$ determined spectroscopically for the primary.

PTFEB132.707+19.810 we analyze both the parameters from Kraus et al. (2017) and those from Gillen et al. (2017). The results are given in Table 13 for HAT-TR-318-007, CM Dra, Kepler-16, and LP 661-13, in Table 14 for WOCS 23009 and KOI-126, and in Table 15 for PTFEB132.707+19.810.

HAT-TR-318-007: To fit the Dartmouth model to the data for HAT-TR-318-007, we vary four parameters: the masses of the two stars, the age of the system, and the metallicity of the system, fitting these to the observed masses and radii of the components and the observed metallicity of the system. The resulting radii are within $\sim 1 \sigma$ of the measured values. The best-fit model has $\chi^{2}=1.3$, and as there is one degree of freedom in this fit, this indicates an excellent fit. We conclude that the masses, radii, and metallicity of HAT-TR-318-007 are consistent with the Dartmouth model. This modeling yields a $95 \%$ confidence lower limit on the age of $t>6.6 \mathrm{Gyr}$. While the Dartmouth model reproduces the masses and radii of the stars, it predicts somewhat hotter temperatures for both components $\left(T_{\text {eff, }}=3487_{-22}^{+29} \mathrm{~K}\right.$ and $\left.T_{\text {eff, } \mathrm{B}}=3255_{-21}^{+25} \mathrm{~K}\right)$ than what we infer from the spectra $\left(T_{\text {eff, }}=3190 \pm 110 \mathrm{~K}\right.$ and $T_{\text {eff, } \mathrm{B}}=3100 \pm 110 \mathrm{~K}$ ). If we include the temperatures as additional observables to be fit by the model, we find a minimum $\chi^{2}=5.56$, with three degrees of freedom. In other words, the data and model are still consistent when the effective temperatures are included in the fit, but the quality of the fit is somewhat poorer. In this case the $95 \%$ confidence lower limit on the age is $t>6.6 \mathrm{Gyr}$.

CM Dra: Fitting the model without including the temperatures yields $[\mathrm{Fe} / \mathrm{H}]=+0.14 \pm 0.06$ and age $=13.07_{-1.22}^{+0.56}$. A high metallicity is required to fit the relatively large radii of these stars, but this is inconsistent with the observed value of $[\mathrm{Fe} / \mathrm{H}]=-0.30 \pm 0.12$. The model radii are $0.9 \sigma$ and $1.7 \sigma$ 
Table 13

Results from Fitting Low-mass Eclipsing Binary Systems with the Dartmouth Stellar Evolution Isochrones

\begin{tabular}{|c|c|c|c|c|}
\hline Parameter & Observed Value & Model Value ${ }^{\mathrm{a}}$ & $(O-\mathrm{C}) / \sigma^{\mathrm{b}}$ & $100 \% \times(O-\mathrm{C}) / O^{\mathrm{c}}$ \\
\hline \multicolumn{5}{|c|}{ HAT-TR-318-007 } \\
\hline$M_{\mathrm{A}}^{\star \dagger}\left(\mathcal{M}_{\odot}^{\mathrm{N}}\right)$ & $0.448 \pm 0.011$ & $0.4665_{-0.0040}^{+0.0047}$ & -1.68 & $-4.13 \%$ \\
\hline$M_{\mathrm{B}}^{\star, \dagger}\left(\mathcal{M}_{\odot}^{\mathrm{N}}\right)$ & $0.2721_{-0.0042}^{+0.0041}$ & $0.2732 \pm 0.0029$ & -0.26 & $-0.40 \%$ \\
\hline$R_{\mathrm{A}}^{\dagger}\left(\mathcal{R}_{\odot}^{\mathrm{N}}\right)$ & $0.4548_{-0.0036}^{+0.0035}$ & $0.4527_{-0.0033}^{+0.0035}$ & 0.58 & $0.24 \%$ \\
\hline$R_{\mathrm{B}}^{\dagger}\left(\mathcal{R}_{\odot}^{\mathrm{N}}\right)$ & $0.2913_{-0.0024}^{+0.0023}$ & $0.2906 \pm 0.0021$ & 0.29 & $0.24 \%$ \\
\hline$T_{\text {eff,A }}(\mathrm{K})$ & $3190 \pm 110$ & $3487_{-22}^{+29}$ & -2.7 & $-9.31 \%$ \\
\hline$T_{\text {eff,B }}(\mathrm{K})$ & $3100 \pm 110$ & $3255_{-21}^{+25}$ & -1.41 & $-5.00 \%$ \\
\hline $\mathrm{Age}^{\star}(\mathrm{Gyr})$ & $\cdots$ & $11.4_{-2.8}^{+1.7}$ & $\cdots$ & $\cdots$ \\
\hline$[\mathrm{Fe} / \mathrm{H}]^{\star, \dagger}(\operatorname{dex})$ & $+0.298 \pm 0.080$ & $+0.330_{-0.080}^{+0.071}$ & -0.40 & $-10.74 \%$ \\
\hline$\chi^{2}(\mathrm{dof})^{\mathrm{d}}$ & $\ldots$ & $1.3(1)$ & $\cdots$ & $\cdots$ \\
\hline \multicolumn{5}{|c|}{ CM Dra } \\
\hline$M_{\mathrm{A}}^{\star, \dagger}\left(\mathcal{M}_{\odot}^{\mathrm{N}}\right)$ & $0.2310 \pm 0.0009$ & $0.23129 \pm 0.00087$ & -0.32 & $-0.13 \%$ \\
\hline$M_{\mathrm{B}}^{\star, \dagger}\left(\mathcal{M}_{\odot}^{\mathrm{N}}\right)$ & $0.2141 \pm 0.0010$ & $0.21515 \pm 0.00092$ & -1.1 & $-0.49 \%$ \\
\hline$R_{\mathrm{A}}^{\dagger}\left(\mathcal{R}_{\odot}^{\mathrm{N}}\right)$ & $0.2534 \pm 0.0019$ & $0.2516 \pm 0.0013$ & 0.95 & $0.71 \%$ \\
\hline$R_{\mathrm{B}}^{\dagger}\left(\mathcal{R}_{\odot}^{\mathrm{N}}\right)$ & $0.2396 \pm 0.0015$ & $0.2371 \pm 0.0011$ & 1.7 & $1.04 \%$ \\
\hline$T_{\text {eff, } \mathrm{A}}(\mathrm{K})$ & $3130 \pm 70$ & $3271 \pm 21$ & -2.0 & $-4.50 \%$ \\
\hline$T_{\text {eff,B }}(\mathrm{K})$ & $3120 \pm 70$ & $3253 \pm 20$ & -1.9 & $-4.26 \%$ \\
\hline Age $^{\star}(\mathrm{Gyr})$ & $\cdots$ & $13.07_{-1.22}^{+0.56}$ & $\cdots$ & $\cdots$ \\
\hline$[\mathrm{Fe} / \mathrm{H}]^{\star, \dagger}(\mathrm{dex})$ & $-0.30 \pm 0.12$ & $+0.14 \pm 0.06$ & -3.7 & $147 \%$ \\
\hline$\chi^{2}(\mathrm{dof})$ & $\ldots$ & $16.81(1)$ & $\cdots$ & $\cdots$ \\
\hline \multicolumn{5}{|c|}{ Kepler-16 } \\
\hline$M_{\AA}^{\star, \dagger}\left(\mathcal{M}_{\odot}^{\mathrm{N}}\right)$ & $0.6897 \pm 0.0035$ & $0.6888_{-0.0038}^{+0.0035}$ & 0.26 & $0.13 \%$ \\
\hline$M_{\mathrm{B}}^{\star \dagger}\left(\mathcal{M}_{\odot}^{\mathrm{N}}\right)$ & $0.20255 \pm 0.00066$ & $0.20296_{-0.00061}^{+0.00068}$ & -0.62 & $-0.20 \%$ \\
\hline$R_{\mathrm{A}}^{\dagger}\left(\mathcal{R}_{\odot}^{\mathrm{N}}\right)$ & $0.6489 \pm 0.0014$ & $0.6492_{-0.0015}^{+0.0014}$ & -0.21 & $-0.05 \%$ \\
\hline$R_{\mathrm{B}}^{\dagger}\left(\mathcal{R}_{\odot}^{\mathrm{N}}\right)$ & $0.22623 \pm 0.00059$ & $0.22588_{-0.00063}^{+0.00059}$ & 0.59 & $0.15 \%$ \\
\hline$T_{\text {eff, } \mathrm{A}}^{\dagger}(\mathrm{K})$ & $4450 \pm 150$ & $4139_{-16}^{+14}$ & 2.1 & $6.99 \%$ \\
\hline$T_{\text {eff,B }}(\mathrm{K})$ & $\cdots$ & $3168 \pm 13$ & $\cdots$ & $\cdots$ \\
\hline $\operatorname{Age}^{\star}(\mathrm{Gyr})$ & $\cdots$ & $3.90_{-0.78}^{+0.85}$ & $\ldots$ & $\ldots$ \\
\hline$[\mathrm{Fe} / \mathrm{H}]^{\star, \dagger}(\mathrm{dex})$ & $-0.30 \pm 0.20$ & $+0.392 \pm 0.045$ & -3.5 & $231 \%$ \\
\hline$\chi^{2}(\mathrm{dof})$ & $\ldots$ & $17.1(2)$ & $\ldots$ & $\ldots$ \\
\hline \multicolumn{5}{|c|}{ LP 661-13 } \\
\hline$M_{\mathrm{A}}^{\star, \dagger}\left(\mathcal{M}_{\odot}^{\mathrm{N}}\right)$ & $0.30795 \pm 0.00084$ & $0.30833_{-0.00082}^{+0.00077}$ & -0.45 & $-0.12 \%$ \\
\hline$M_{\mathrm{B}}^{\star, \dagger}\left(\mathcal{M}_{\odot}^{\mathrm{N}}\right)$ & $0.19400 \pm 0.00034$ & $0.19403 \pm 0.00035$ & -0.088 & $-0.02 \%$ \\
\hline$R_{\mathrm{A}}^{\dagger}\left(\mathcal{R}_{\odot}^{\mathrm{N}}\right)$ & $0.3226 \pm 0.0033$ & $0.3147 \pm 0.0015$ & 2.4 & $2.45 \%$ \\
\hline$R_{\mathrm{B}}^{\dagger}\left(\mathcal{R}_{\odot}^{\mathrm{N}}\right)$ & $0.2174 \pm 0.0023$ & $0.2165 \pm 0.0012$ & 0.39 & $0.41 \%$ \\
\hline$T_{\text {eff,A }}(\mathrm{K})$ & $\cdots$ & $3364 \pm 26$ & $\cdots$ & $\cdots$ \\
\hline$T_{\text {eff,B }}(\mathrm{K})$ & $\cdots$ & $3243 \pm 21$ & $\cdots$ & $\cdots$ \\
\hline $\mathrm{Age}^{\star}(\mathrm{Gyr})$ & $\ldots$ & $12.50_{-1.7}^{+0.94}$ & $\ldots$ & $\cdots$ \\
\hline$[\mathrm{Fe} / \mathrm{H}]^{\star, \dagger}(\mathrm{dex})$ & $-0.07 \pm 0.1$ & $+0.092 \pm 0.072$ & -1.6 & $231 \%$ \\
\hline$\chi^{2}(\mathrm{dof})$ & $\cdots$ & $7.7(1)$ & $\cdots$ & $\cdots$ \\
\hline
\end{tabular}

Notes. HAT-TR-318-007, CM Dra, Kepler-16, and LP 661-13. Parameters marked by a ${ }^{\star}$ are varied in the fit. Parameters marked by a ${ }^{\dagger}$ are treated as observables that are matched to the model in computing the value of $\chi^{2}$ that is listed.

${ }^{a}$ The optimized value and uncertainty for this parameter that come from the analysis in Section 4.3. The uncertainties do not include any systematic errors in the stellar evolution models.

${ }^{b}$ Difference between the observed parameter value and the model value, divided by the observational uncertainty.

${ }^{c}$ Difference between the observed parameter value and the model value, expressed as a percentage of the observed parameter value.

d The $\chi^{2}$ for the best-fit model. The number of degrees of freedom in the analysis is listed in parentheses following the $\chi^{2}$ value.

smaller $(0.7 \%$ and $1 \%)$ than the measured radii of the primary and secondary, respectively. The resulting $\chi^{2}$ for the best-fit model is 16.81 , which has a $4 \times 10^{-5}$ probability of occurring by chance when there is one degree of freedom. If the metallicity is fixed to $[\mathrm{Fe} / \mathrm{H}]=-0.30$, then the model radii are $4.5 \sigma$ and $6.9 \sigma(3.4 \%$ and $4.0 \%)$ too small. The model temperatures ( 3270 and $3250 \mathrm{~K}$ ) are also somewhat larger than the observed values $(3130 \pm 70 \mathrm{~K}$ and $3120 \pm 70 \mathrm{~K})$. Including 
Table 14

Results from Fitting Low-mass Eclipsing Binary Systems with the Dartmouth Stellar Evolution Isochrones

\begin{tabular}{|c|c|c|c|c|}
\hline Parameter & Observed Value & Model Value $^{\mathrm{a}}$ & $(O-C) / \sigma^{\mathrm{b}}$ & $100 \% \times(O-\mathrm{C}) / O$ \\
\hline \multicolumn{5}{|c|}{ WOCS $23009^{d}$} \\
\hline$M_{\mathrm{A}}^{\star}\left(\mathcal{M}_{\odot}^{\mathrm{N}}\right)$ & $\ldots$ & $1.462_{-0.042}^{+0.027}$ & $\ldots$ & $\cdots$ \\
\hline$R_{\mathrm{A}}\left(\mathcal{R}_{\odot}^{\mathrm{N}}\right)$ & $\cdots$ & $2.136_{-0.025}^{+0.015}$ & $\cdots$ & $\cdots$ \\
\hline$R_{\mathrm{B}}\left(\mathcal{R}_{\odot}^{\mathrm{N}}\right)$ & $\ldots$ & $0.4292_{-0.0050}^{+0.0030}$ & $\ldots$ & $\cdots$ \\
\hline$T_{\mathrm{eff}, \mathrm{A}}^{\dagger}(\mathrm{K})$ & $6320 \pm 150$ & $6295_{-130}^{+100}$ & 0.17 & $0.40 \%$ \\
\hline$K_{\mathrm{A}}^{\dagger}\left(\mathrm{km} \mathrm{s}^{-1}\right)$ & $6.96 \pm 0.13$ & $7.075_{-0.038}^{+0.052}$ & -0.88 & $-1.65 \%$ \\
\hline$R_{\mathrm{A}} / R_{\mathrm{B}}^{\dagger}$ & $4.977 \pm 0.009$ & $4.9782_{-0.0095}^{+0.0086}$ & -0.13 & $-0.02 \%$ \\
\hline$\left(R_{\mathrm{A}}+R_{\mathrm{B}}\right) / a^{\dagger}$ & $0.005836 \pm 0.000020$ & $0.005829 \pm 0.000021$ & 0.35 & $0.12 \%$ \\
\hline $\operatorname{Age}^{\star, \dagger}(\mathrm{Gyr})$ & $2.62 \pm 0.25$ & $2.68_{-0.26}^{+0.34}$ & -0.24 & $-2.29 \%$ \\
\hline$[\mathrm{Fe} / \mathrm{H}]^{\star, \dagger}(\mathrm{dex})$ & $+0.09 \pm 0.03$ & $+0.09 \pm 0.03$ & 0.0 & $0.00 \%$ \\
\hline$\chi^{2}(\mathrm{dof})^{\mathrm{e}}$ & $\cdots$ & $0.83(2)$ & $\cdots$ & $\cdots$ \\
\hline$M_{C}^{\star \dagger \dagger}\left(\mathcal{M}_{\odot}^{\mathrm{N}}\right)$ & $0.2127 \pm 0.0026$ & $0.2139_{-0.0016}^{+0.0014}$ & -0.46 & $-0.56 \%$ \\
\hline$R_{\mathrm{A}}^{\dagger}\left(\mathcal{R}_{\odot}^{\mathrm{N}}\right)$ & $2.0254 \pm 0.0098$ & $2.0250_{-0.0102}^{+0.0093}$ & 0.041 & $0.02 \%$ \\
\hline$R_{\mathrm{B}}^{\dagger}\left(\mathcal{R}_{\odot}^{\mathrm{N}}\right)$ & $0.2543 \pm 0.0014$ & $0.2543 \pm 0.0013$ & 0.0 & $0.00 \%$ \\
\hline$R_{C}^{\dagger}\left(\mathcal{R}_{\odot}^{\mathrm{N}}\right)$ & $0.2318 \pm 0.0013$ & $0.2317 \pm 0.0010$ & 0.077 & $0.04 \%$ \\
\hline$T_{\mathrm{eff}, \mathrm{A}}^{\dagger}(\mathrm{K})$ & $5875 \pm 100$ & $5980_{-78}^{+73}$ & -1.1 & $-1.79 \%$ \\
\hline$T_{\text {eff,B }}(\mathrm{K})$ & $\cdots$ & $3255_{-19}^{+17}$ & $\cdots$ & $\cdots$ \\
\hline$T_{\text {eff,C }}(\mathrm{K})$ & $\cdots$ & $3227_{-18}^{+16}$ & $\cdots$ & $\cdots$ \\
\hline Age $^{\star}(\mathrm{Gyr})$ & $\cdots$ & $3.71_{-0.19}^{+0.41}$ & $\cdots$ & $\cdots$ \\
\hline$[\mathrm{Fe} / \mathrm{H}]^{\star, \dagger}(\operatorname{dex})$ & $0.15 \pm 0.08$ & $0.221_{-0.054}^{+0.060}$ & -0.89 & $-47 \%$ \\
\hline$\chi^{2}(\mathrm{dof})$ & $\cdots$ & $2.2(3)$ & $\ldots$ & $\cdots$ \\
\hline
\end{tabular}

Notes. WOCS 23009 and KOI-126. Parameters marked by a * are varied in the fit. Parameters marked by a ${ }^{\dagger}$ are treated as observables that are matched to the model in computing the value of $\chi^{2}$ that is listed.

${ }^{a}$ The optimized value and uncertainty for this parameter that come from the analysis in Section 4.3. The uncertainties do not include any systematic errors in the stellar evolution models.

${ }^{b}$ Difference between the observed parameter value and the model value, divided by the observational uncertainty.

${ }^{c}$ Difference between the observed parameter value and the model value, expressed as a percentage of the observed parameter value.

d WOCS 23009 is a single-lined eclipsing binary system, but a member of the open cluster NGC 6819. The published masses and radii of the components of this system are inferred from the Dartmouth isochrones, so we do not list these as "observed" values.

${ }^{\mathrm{e}}$ The $\chi^{2}$ for the best-fit model. The number of degrees of freedom in the analysis is listed in parentheses following the $\chi^{2}$ value.

the temperatures as observables in the fit yields a similar result. We note that Feiden \& Chaboyer (2014) find that the Darmouth evolution models and observations can be reconciled for CM Dra by invoking a $\sim 0.2$ dex $\alpha$-element enhancement, near-solar metallicity, and an age (based partly on the cooling age of the white dwarf companion) of $8.5 \pm 3.5 \mathrm{Gyr}$.

WOCS 23009: This long-period single-lined binary is a member of the open cluster NGC 6819. The color-magnitude diagrams for the cluster, together with multiple eclipsing binaries, enable a precise determination of the cluster age of $2.62 \pm 0.25$ Gyr. Additionally, the metallicity has been precisely determined to be $[\mathrm{Fe} / \mathrm{H}]=+0.09 \pm 0.03$. Sandquist et al. (2013) have previously shown that the Dartmouth models are consistent with the observed properties of WOCS 23009. We repeat this comparison but within the framework presented in this section to allow a fair quantitative comparison with the other systems. In this case we treat the following parameters as observables to be matched by the model: the effective temperature of the primary $T_{\text {eff, } 1}=6320 \pm 150 \mathrm{~K}$, the semiamplitude of the primary star's
$\mathrm{RV}$ orbit $K=6.96 \pm 0.13 \mathrm{~km} \mathrm{~s}^{-1}$, the ratio of the radii $R_{1} / R_{2}=4.977 \pm 0.009$, the sum of the radii relative to the semimajor axis $\left(R_{1}+R_{2}\right) / a=0.005836 \pm 0.000020$, the metallicity $[\mathrm{Fe} / \mathrm{H}]=+0.09 \pm 0.03$, and the age of the cluster $2.62 \pm 0.25$ Gyr. We vary the masses of the two component stars and the age and metallicity of the isochrones in our fit. We find that the best-fit model has $\chi^{2}=0.83$, which, given that there are two degrees of freedom, indicates that the model is in excellent agreement with the observations.

KOI-126: This is a triply eclipsing hierarchical triple system discovered by Kepler (Carter et al. 2011). Feiden et al. (2011) have previously shown that the Dartmouth models are in good agreement with the observed masses and radii. As for WOCS 23009, we perform our own modeling of this system using the framework presented here. In this case the observed parameters are the masses and radii of the three component stars, the temperature of the primary star, and the metallicity of the system. These parameters are taken from Carter et al. (2011). The free parameters in the model are the masses of the three component stars, the metallicity, and the age. 
Table 15

Results from Fitting Low-mass Eclipsing Binary Systems with the Dartmouth Stellar Evolution Isochrones

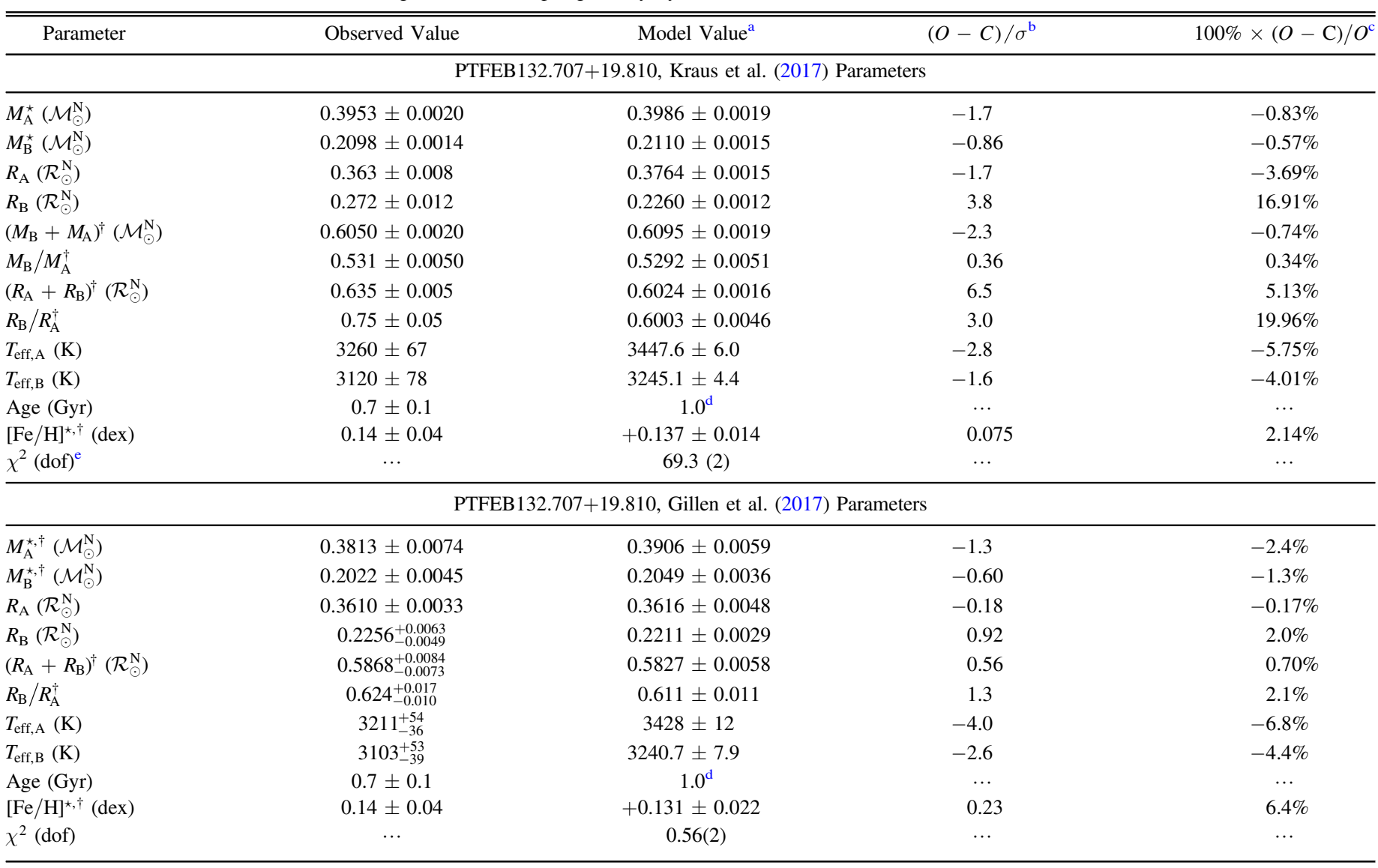

Notes. Parameters marked by a ${ }^{\star}$ are varied in the fit. Parameters marked by a ${ }^{\dagger}$ are treated as observables that are matched to the model in computing the value of $\chi^{2}$ that is listed.

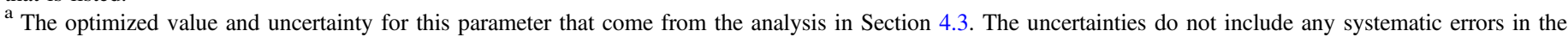
stellar evolution models.

${ }^{\mathrm{b}}$ Difference between the observed parameter value and the model value, divided by the observational uncertainty.

${ }^{c}$ Difference between the observed parameter value and the model value, expressed as a percentage of the observed parameter value.

d The age of PTFEB132.707+19.810 was fixed to $1.0 \mathrm{Gyr}$ in this analysis, which is the youngest age at which the Dartmouth isochrones have been tabulated.

e The $\chi^{2}$ for the best-fit model. The number of degrees of freedom in the analysis is listed in parentheses following the $\chi^{2}$ value.

The best-fit model has $\chi^{2}=2.2$ with three degrees of freedom, indicating a good fit to the observations.

Kepler-16: This is an eclipsing binary system with a transiting circumbinary planet discovered by Doyle et al. (2011). The transiting planet allows the masses and radii of both stars to be determined with high precision from the light curve alone. Additionally, the temperature and metallicity of the primary star, which dominates the light of the system, have been determined spectroscopically. The observables that we attempt to fit are $M_{1}=0.6897 \pm 0.0035 M_{\odot}, R_{1}=0.6489 \pm 0.0014 \mathcal{R}_{\odot}^{\mathrm{N}}, M_{2}=$ $0.20255 \pm 0.00066 M_{\odot}, R_{2}=0.22623 \pm 0.00059 \mathcal{R}_{\odot}^{\mathrm{N}}, T_{\text {eff, } 1}=$ $4450 \pm 150 \mathrm{~K}$, and $[\mathrm{Fe} / \mathrm{H}]=[\mathrm{M} / \mathrm{H}]=-0.3 \pm 0.2$. The free parameters in the model are the masses of the two stars and the age and metallicity of the system. The best-fit model has $\chi^{2}=17.1$ with two degrees of freedom. The probability that such a high value of $\chi^{2}$ is found by chance is only $2 \times 10^{-4}$, so the model does not provide a good fit to the observations within the errors. The model can match the observed masses and radii of the system to within $1 \sigma$, but it requires a metallicity that is $3.5 \sigma(0.69 \mathrm{dex})$ too high to do so. If we fix the metallicity to -0.3 , the predicted radius of the primary is too high by $2.3 \sigma(0.5 \%)$, while the predicted radius of the secondary is too low by $8 \sigma(2.1 \%)$. The model temperature of the primary is also too high by $2.1 \sigma(7 \%)$. The high values for the radius and temperature of the primary are due to the model choosing a large age of $8.5 \mathrm{Gyr}$ to better match the radius of the secondary. If an age is adopted that fits the primary mass and radius, then the secondary radius is too large by $19 \sigma(5 \%)$.

LP 661-13: This is a double-lined $\mathrm{M}$ dwarf eclipsing binary system discovered by Dittmann et al. (2017). The parameters for the primary component are listed in Table 12, while the secondary component has a mass of $0.19400 \pm 0.00034 M_{\odot}$ and a radius of $0.2174 \pm 0.0023 \mathcal{R}_{\odot}^{\mathrm{N}}$. We find that a relatively large age $(12.2 \pm 1.3 \mathrm{Gyr})$ and supersolar metallicity $(+0.09 \pm 0.07 \mathrm{dex})$ are required to fit the observed masses and radii. This modeling yields radii for the primary and secondary components that are too small by $2.4 \sigma(2.4 \%)$ and by $0.4 \sigma(0.4 \%)$, respectively. The resulting $\chi^{2}$ for the best-fit model is 7.7 with one degree of freedom, indicating a marginally acceptable fit (5\% probability of occurring by chance). When the metallicity is fixed to the observed value of 
$-0.07 \mathrm{dex}$, the resulting radii are too small by $3.2 \sigma(3.2 \%)$ and $1.4 \sigma(1.5 \%)$, respectively.

PTFEB132.707+19.810: This is a double-lined $\mathrm{M}$ dwarf eclipsing binary system in the Praesepe cluster discovered by Kraus et al. (2017) and independently by Gillen et al. (2017). We first fit the parameters from Kraus et al. (2017) for the system in a similar manner to that for CM Dra, but in this case we fix the age to $1.0 \mathrm{Gyr}$, the minimum age tabulated in the Dartmouth isochrones, given the estimated age of $600-800 \mathrm{Myr}$ for the cluster. Fitting the model without including the temperatures yields a radius for the primary star that is $1.7 \sigma$ larger than the measured radius and a radius for the secondary star that is $3.8 \sigma$ smaller than the measured radius. The resulting $\chi^{2}$ for the best-fit model is 69.3 with two degrees of freedom, indicating a very poor fit $\left(8.9 \times 10^{-16}\right.$ probability of occurring by chance). The model yields temperatures of 3450 and $3250 \mathrm{~K}$ for the primary and secondary stars that are too large by $2.8 \sigma$ and $1.6 \sigma$, respectively. While the 1.0 Gyr Dartmouth isochrone clearly provides a poor fit to the observed properties of this binary system, we caution that the secondary component may still be contracting onto the main sequence at the younger age of the Praesepe cluster, which may explain the discrepancy. If we instead use the parameters from Gillen et al. (2017), which are based on the same $K 2$ light curve, but different spectroscopy, and exclude the effective temperatures, we find excellent agreement with the models with $\chi^{2}=0.56$ for the best-fit model. MacDonald \& Mullan (2017) also conclude that the Gillen et al. (2017) values are in better agreement with models than the Kraus et al. (2017) parameters.

To summarize the results of our comparison with the Dartmouth models, we find that the masses, radii, metallicities, and ages (when independently known) of the stars in HAT-TR318-007, WOCS 23009, and KOI-126 are well matched by these models, while those of Kepler-16 are not. For CM Dra the results are not consistent if we assume a subsolar $[\mathrm{Fe} / \mathrm{H}]$ as reported by Terrien et al. (2012b) (see, however, Feiden \& Chaboyer 2014), while the Kraus et al. (2017) parameters for PTFEB132.707+19.810 are inconsistent with the models, but the Gillen et al. (2017) values are in agreement with the models. The observations of LP 661-13 are in slight disagreement with the models at the $\sim 2 \sigma$ level. We note that the three systems that are in agreement with the models are older than 1 Gyr (or at least do not have independent age determinations, indicating that they are younger than this) and have supersolar metallicities. The other systems either have subsolar metallicities (CM Dra, Kepler-16, and LP 661-13) or are younger than 1 Gyr (PTFEB132.707+19.810), and in two cases have conflicting parameter values, some of which are consistent with the models and some of which are not (CM Dra, PTFEB132.707 $+19.810)$.

A similar conclusion that subsolar-metallicity stars are not well matched by the Dartmouth models, at least for fully convective stars, was reached by Feiden \& Chaboyer (2013) in the context of testing magnetic models. It is not clear whether the agreement with the models for the higher-metallicity systems is fortuitous. Since both increased age and enhanced metallicity tend to allow for larger radii, if these binary components are actually inflated owing to stellar activity, then perhaps we should expect to see better agreement with the models for metal-rich stars, especially when they are allowed to have old ages. If that is the case, then we should not expect the age inferred for HAT-TR-318-007 to be accurate. While the radii of the high-metallicity stars are in agreement with the models, the temperatures are systematically too low. This is also seen for other stars where the metallicities have not been determined. While the measured masses and radii are largely model independent, ${ }^{13}$ the measured temperatures depend on theoretical atmosphere models. Therefore, we cannot say whether the disagreement between the measured and expected temperatures is due to errors in the stellar evolution models, in the atmosphere and spectral synthesis models used in measuring the temperatures, or both.

\subsection{Summary}

In this paper we have presented the discovery of a new double-lined $\mathrm{M}$ dwarf binary with total secondary eclipses. The results can be summarized as follows:

1. By combining optical radial velocity measurements for both components with photometric observations of the eclipses, we measure the masses and radii of both stars to be $M_{\mathrm{A}}=0.448 \pm 0.011 \mathcal{M}_{\odot}^{\mathrm{N}}, M_{\mathrm{B}}=0.2721_{-0.0042}^{+0.0041} \mathcal{M}_{\odot}^{\mathrm{N}}$, $R_{\mathrm{A}}=0.4548_{-0.0036}^{+0.0035} \mathcal{R}_{\odot}^{\mathrm{N}}$, and $R_{\mathrm{B}}=0.2913_{-0.0024}^{+0.0023} \mathcal{R}_{\odot}^{\mathrm{N}}$.

2. We find that the system has a small, but significant, nonzero eccentricity of $0.0136 \pm 0.0026$.

3 . The $K 2$ observations show a strikingly coherent nearly sinusoidal variation with a period of $3.41315_{-0.00032}^{+0.00030}$ days, which is slightly longer than the orbital period. We demonstrate that the signal is due to the primary star and interpret it as the rotation period of this component. The slight asynchronicity might be due to differential rotation, or a magnetized wind that balances the torque from tides. Ground-based $r$ - and $i$-band light curves obtained many years before $K 2$ show no evidence of this variation, with limits on the amplitude that are several times lower what was seen in $K 2$ (by nearly a factor of 10 in the case of the HATNet $r$ band).

4. The $K 2$ observations show an additional modulation at a period of $3.28498_{-0.00034}^{+0.00035}$ days (with two peaks per cycle, or a near-sinusoidal variation at half this value). We cannot determine whether the signal is due to the primary or secondary component.

5. We obtained NIR spectra of the system during total eclipse, and near both quadrature phases, and used these observations to disentangle the spectra of the two components.

6. Based on the disentangled spectra, we measure metallicities and effective temperatures for the two components of $T_{\text {eff, } \mathrm{A}}=3190 \pm 110 \mathrm{~K}, T_{\text {eff,B }}=3100 \pm 110 \mathrm{~K}$, $[\mathrm{Fe} / \mathrm{H}]_{\mathrm{A}}=+0.40 \pm 0.11$, and $[\mathrm{Fe} / \mathrm{H}]_{\mathrm{B}}=+0.20 \pm 0.11$, or a metallicity of $[\mathrm{Fe} / \mathrm{H}]=+0.298 \pm 0.080$ for the system if we assume that the two stars have the same abundances. We find consistent results when using empirically calibrated spectral indices and when cross-correlating the spectra against BT-Settl synthetic templates.

7. The space velocity of the system indicates that it is a member of the Galactic disk.

8. We carried out tests that indicate that the total eclipsing nature of this system significantly improves the accuracy with which the parameters may be measured.

9. We find that the masses and radii of the stars in this system are well matched by the Dartmouth stellar evolution models for a system age of $t>6.6 \mathrm{Gyr}$. We also find that these same models reproduce $M$ dwarfs in two other

\footnotetext{
$\overline{13}$ That is, they depend only on very well understood and accepted Keplerian physics.
} 
systems (WOCS 23009B and KOI-126B+C) in the mass range $0.2 \mathcal{M}_{\odot}^{\mathrm{N}}<M<0.5 \mathcal{M}_{\odot}^{\mathrm{N}}$ with well-measured masses and radii and supersolar metallicities, but do not match two other systems with subsolar-metallicity stars (Kepler-16B, LP 661-13A). There are two systems with conflicting sets of measured parameters, some of which are in agreement with the models and some of which are not (CM Dra, PTFEB132.707+19.810).

Further improvement in the precision of the parameter estimates for this system will require higher-precision RV measurements. At $V \sim 16 \mathrm{mag}$, the star is quite faint and pushes the limits of the FLWO $1.5 \mathrm{~m}$ telescope used to obtain the RVs presented here. More precise measurements will require a larger telescope. The treatment of starspots could also be improved. In particular, the $K 2$ light curve may allow spots to be mapped on the surfaces of the component stars.

Partial support for the work reported here was provided by NASA grants NNX09AB29G, NNX13AJ15G, NNX14AE87G, and NNX17AB61G and NSF grant AST-1108686. G.T. acknowledges partial support for this work from NSF grant AST-1509375. Some of the data presented in this paper were obtained from the Mikulski Archive for Space Telescopes (MAST). STScI is operated by the Association of Universities for Research in Astronomy, Inc., under NASA contract NAS5-26555. Support for MAST for non-HST data is provided by the NASA Office of Space Science via grant NNX09AF08G and by other grants and contracts. This paper includes data collected by the Kepler mission. Funding for the Kepler mission is provided by the NASA Science Mission directorate.

\section{Appendix A Correcting for Light-travel Time within the HAT-TR-318-007 System}

The light-travel-time correction for a star to the center of mass of the system is given by

$$
\Delta t=z / c,
$$

where $z$ is the line-of-sight component of the star's barycentric orbit and $c$ is the speed of light. Following Hilditch (2001), this can be expressed in terms of the eccentric anomaly $E$ via

$$
\Delta t=\frac{a}{c}\left[\sqrt{1-e^{2}} \sin E \cos \omega+(\cos E-e) \sin \omega\right] \sin i,
$$

where $a$ is the semimajor axis of the star's orbit about the center of mass of the system, and a factor of -1 appears in front of the right-hand side of the equation for the secondary star. The relation between the time of observation from the solar system barycenter $t$ and the appropriate $E$ to use for describing a star's position is given by Kepler's equation corrected for $\Delta t$ :

$$
E-e \sin E=\frac{2 \pi}{P}[(t-\Delta t)-T],
$$

where the reference time $T$ is the time of periastron passage in the system barycenter frame and $P$ is the orbital period. Given a value of $t$, we solve for $E$ using a Newton-Raphson procedure. The radial velocities for stars 1 and 2 at time $t$ are then determined from $E_{1}$ and $E_{2}$ using standard formulae.
Given anomalies $E_{1,2}$, the sky-projected $x_{1,2}$ and $y_{1,2}$ positions for stars 1 and 2 are then determined from

$$
\begin{gathered}
x_{i}=(-1)^{i-1} a_{i}\left(1-e \cos E_{i}\right) \cos \left(\theta_{i}+\omega\right) \\
y_{i}=(-1)^{i-1} a_{i}\left(1-e \cos E_{i}\right) \sin \left(\theta_{i}+\omega\right) \cos i,
\end{gathered}
$$

where $\theta$ and $E$ are related via

$$
\begin{gathered}
\cos \theta_{i}=\left(\cos E_{i}-e\right) /\left(1-e \cos E_{i}\right) \\
\sin \theta_{i}=\sqrt{1-e^{2}} \sin E_{i} /\left(1-e \cos E_{i}\right) .
\end{gathered}
$$

The sky-projected separation between the two stars at observed time $t$ is then given by

$$
\rho=\sqrt{\left(x_{1}-x_{2}\right)^{2}+\left(y_{1}-y_{2}\right)^{2}} .
$$

The observed flux for the system can be determined at time $t$ by finding time $\tilde{t}$ such that the $\tilde{\rho}(\tilde{t})=\rho(t)$ and using $\tilde{t}$ as input to the JKTEBOP routine. Here $\tilde{\rho}$ is the sky-projected separation calculated without accounting for intrasystem light-travel time. Note that the time correction is not properly handled for proximity effects such as tidal distortion or the reflection effect; however, these effects are negligible for the well-detached HAT-TR-318-007 system.

For our analysis we determine the $\tilde{t}$ values one time for all photometric observations of HAT-TR-318-007 and then use these as the fixed times of observation throughout our fitting procedure. While a proper treatment would determine a new set of $\tilde{t}$ values for each set of system parameters in the Markov Chain, in practice the uncertainties on $t-\tilde{t}$ due to uncertainties in the system parameters are much less than our timing precision for the system, so this level of complexity, which substantially slows the analysis, is not required.

\section{Appendix B \\ Details of Spectral Index Calculations}

Spectral types for both components of HAT-TR-318-007 were determined using the $\mathrm{H}_{2} \mathrm{O}-\mathrm{K}$ index following Rojas-Ayala et al. (2012). When applied to the disentangled spectra, this yields spectral types of M3.7 \pm 0.7 and M5.0 \pm 0.7 for the primary and secondary stars, respectively. When the index is calculated on the three spectra obtained during total eclipse, the resulting spectral type is M3.6 \pm 0.7 . The uncertainties here include a systematic uncertainty of \pm 0.5 as given by Rojas-Ayala et al. (2012) and an uncertainty in our measurement of the index as described below. As a validation of our spectral type estimates, we also obtained FIRE observations for a number of $\mathrm{M}$ dwarf spectral standards. Figure 19 compares the spectral types estimated from our FIRE observations of these stars to the literature values, demonstrating agreement to $\sim \pm 0.5$ spectral types over the range from M1 to M9. Using the relation between spectral type and effective temperature given in Bessell (1991), we estimate effective temperatures of the component stars of $T_{\mathrm{eff}, \mathrm{A}}=3200 \pm 120 \mathrm{~K}$ and $T_{\mathrm{eff}, \mathrm{B}}=3000 \pm$ $130 \mathrm{~K}$.

Between $\mathrm{T} 12$ and R12 there are four separate NIR metallicity indicators. These include the T12 $\mathrm{H}$ - and $\mathrm{K}$-band $[\mathrm{Fe} / \mathrm{H}]$ indicators $\left([\mathrm{Fe} / \mathrm{H}]_{H, T 12}\right.$ and $[\mathrm{Fe} / \mathrm{H}]_{K, T 12}$, respectively) and the $\mathrm{R} 12 K$-band $[\mathrm{Fe} / \mathrm{H}]$ and $[\mathrm{M} / \mathrm{H}]$ indicators $\left([\mathrm{Fe} / \mathrm{H}]_{\mathrm{R} 12}\right.$ and $\left.[\mathrm{M} / \mathrm{H}]_{\mathrm{R} 12}\right)$. The $[\mathrm{Fe} / \mathrm{H}]_{K, T 12},[\mathrm{Fe} / \mathrm{H}]_{\mathrm{R} 12}$, and $[\mathrm{M} / \mathrm{H}]_{\mathrm{R} 12}$ are not independent indicators in the sense that they make use of the same spectral features; $[\mathrm{Fe} / \mathrm{H}]_{H, T 12}$, on the other hand, is independent of the other three indicators. We calculated each of these metallicity indicators for each of our spectra. 


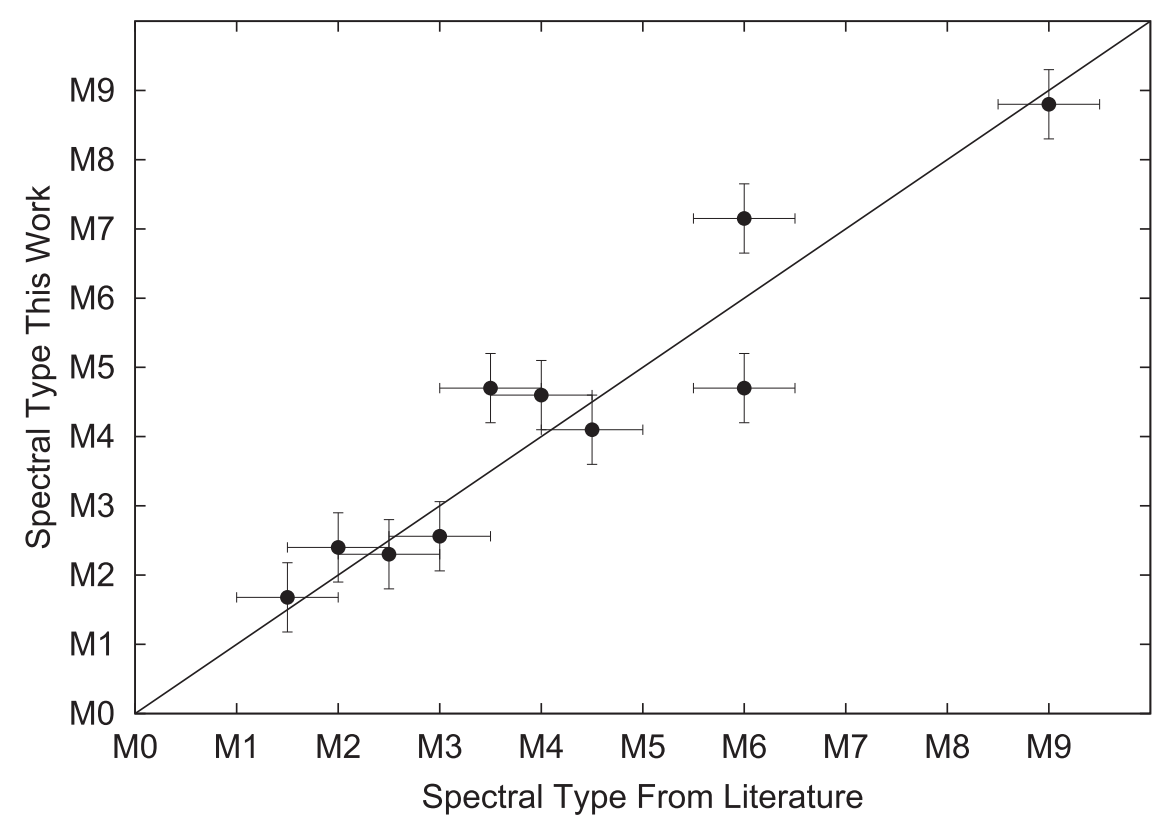

Figure 19. Comparison between spectral types for $\mathrm{M}$ dwarf standards determined from our FIRE/Magellan observations using the $\mathrm{R} 12 \mathrm{H}_{2} \mathrm{O}-\mathrm{K}$ index and spectral types taken from the literature.

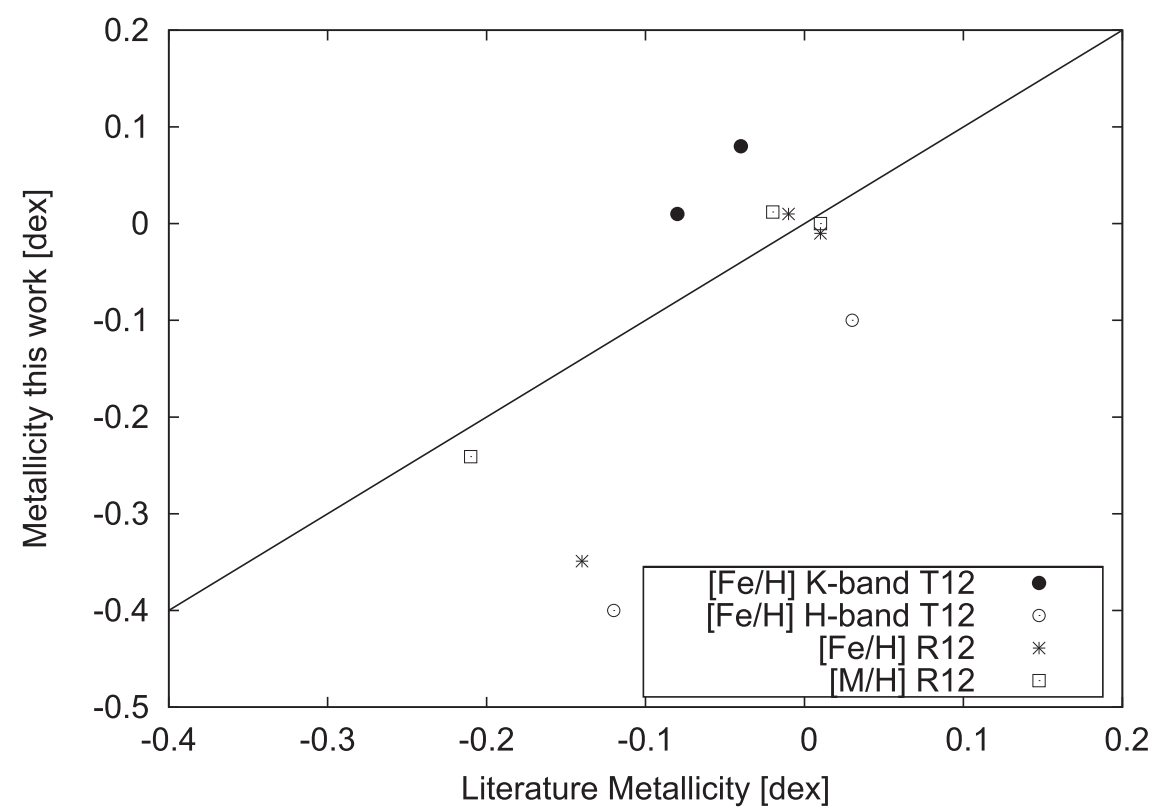

Figure 20. Comparison between the metallicities determined from our observations and metallicities given in the literature for three objects with prior metallicity determinations. We compare each type of metallicity $\left([\mathrm{Fe} / \mathrm{H}]_{H, T 12},[\mathrm{Fe} / \mathrm{H}]_{K, T 12},[\mathrm{Fe} / \mathrm{H}]_{\mathrm{R} 12}\right.$, and $\left.[\mathrm{M} / \mathrm{H}]_{\mathrm{R} 12}\right)$ that is available.

To determine the uncertainties on these indices, we first estimated formal errors for each index by propagating the uncertainties in the spectra based on photon-counting statistics through the index calculations. For each index we then determined a systematic error in precision using a likelihood function of the form

$$
\begin{aligned}
\ln L= & \sum_{j} \sum_{i}-\frac{1}{2}\left[\operatorname { l n } \left(\alpha^{2}\right.\right. \\
& \left.\left.+\sigma_{j i}^{2}\right)+\left(X_{j i}-\bar{X}_{j}\right)^{2} /\left(\alpha^{2}+\sigma_{j i}^{2}\right)\right]
\end{aligned}
$$

(i.e., we assume a standard Gaussian probability distribution), where the sum on $j$ is over stars, the sum on $i$ is over individual measurements for each star, $\alpha$ is the systematic uncertainty for the index, $\sigma_{j i}$ is the formal uncertainty for the $i$ th observation of the $j$ th star, $X_{j i}$ is the measured value of the index, and $\bar{X}_{j}$ is the estimated value for star $j$. We carry out an MCMC analysis varying $\alpha$ and $\bar{X}_{j}$ to determine optimal values and uncertainties for these parameters. The value of $\alpha$, determined in this manner, represents the excess scatter in the measurements for an individual star beyond what is expected based on the formal errors. In addition to this, there are possible errors in comparing our indices 
to those given by T12 and R12 (and thus in using our indices directly in their relations), and there are additional systematic errors in the relations given by $\mathrm{T} 12$ and R12 in going from spectral metallicity indices to physical metallicities.

There are a total of three objects with metallicities given in either of these catalogs for which we have obtained observations (two of the objects are in both T12 and R12, while one object is in R12 only). Due to this small number of calibrators, we do not attempt to derive an independent metallicity calibration from our observations. Figure 20 compares the metallicity indices for these stars from our observations to those given in T12 and R12. We find that an additional scatter in the metallicity indices of \pm 0.062 dex must be added in quadrature to the formal uncertainties such that $\chi^{2}$ per degree of freedom is unity. On top of this, T12 and R12 give estimates of the uncertainties in the physical metallicities inferred from these indices of $\pm 0.12 \mathrm{dex}$ for $[\mathrm{Fe} / \mathrm{H}]_{H, T 12}, \pm 0.12$ dex for $[\mathrm{Fe} / \mathrm{H}]_{K, T 12}, \pm 0.14$ dex for $[\mathrm{Fe} / \mathrm{H}]_{\mathrm{R} 12}$, and \pm 0.10 dex for $[\mathrm{M} / \mathrm{H}]_{\mathrm{R} 12}$.

From our disentangled spectra of HAT-TR-318-007 we measure $[\mathrm{Fe} / \mathrm{H}]_{H, T 12}=+0.23 \pm 0.19,[\mathrm{Fe} / \mathrm{H}]_{K, T 12}=+0.49 \pm 0.14$, $[\mathrm{Fe} / \mathrm{H}]_{R 12}=+0.55 \pm 0.17$, and $[\mathrm{M} / \mathrm{H}]_{R 12}=+0.39 \pm 0.14$ for the primary star and $[\mathrm{Fe} / \mathrm{H}]_{H, T 12}=+0.43 \pm 0.19$, $[\mathrm{Fe} / \mathrm{H}]_{K, T 12}=+0.07 \pm 0.14, \quad[\mathrm{Fe} / \mathrm{H}]_{R 12}=+0.08 \pm 0.21$, and $[\mathrm{M} / \mathrm{H}]_{R 12}=+0.06 \pm 0.15$ for the secondary star. The error estimates given here include all the sources of uncertainty discussed above. For reference, using the four spectra obtained during totality, we measure $[\mathrm{Fe} / \mathrm{H}]_{H, T 12}=+0.39 \pm 0.16$, $[\mathrm{Fe} / \mathrm{H}]_{K, T 12}=+0.49 \pm 0.14, \quad[\mathrm{Fe} / \mathrm{H}]_{R 12}=+0.63 \pm 0.16$, and $[\mathrm{M} / \mathrm{H}]_{R 12}=+0.45 \pm 0.13$ for the primary. To obtain final estimates for each star, we take the weighted mean of the $[\mathrm{Fe} / \mathrm{H}]_{H, T 12}$ and $[\mathrm{Fe} / \mathrm{H}]_{K, T 12}$ measurements, ${ }^{14}$ finding $[\mathrm{Fe} / \mathrm{H}]_{\mathrm{A}}=+0.40 \pm 0.11$ and $[\mathrm{Fe} / \mathrm{H}]_{\mathrm{B}}=+0.20 \pm 0.11$, which are consistent to within $2 \sigma$. Assuming that both components have the same metallicity, we take the weighted mean of the indi vidual metallicities to estimate a system metallicity of $[\mathrm{Fe} / \mathrm{H}]=+0.298 \pm 0.080$.

\section{Appendix C \\ Details of Cross-correlation against Theoretical Spectral Templates}

As an alternative method to determine the stellar atmospheric parameters, we compare our disentangled NIR spectra to model spectra from the BT-Settl grid (Allard et al. 2011) computed using the Asplund et al. (2009) solar abundances. The models have temperatures between 2600 and $4000 \mathrm{~K}$ in steps of $100 \mathrm{~K}$ and have $[\mathrm{Fe} / \mathrm{H}]$ metallicities between -4.0 and +0.5 dex in $0.5 \mathrm{dex}$ increments. A metallicity of $[\mathrm{Fe} / \mathrm{H}]=+0.3 \mathrm{dex}$ is also included. The models assume solar-scaled abundances with $\alpha$-enhancement for subsolar metallicities such that $[\alpha / \mathrm{Fe}]=+0.2$ for $[\mathrm{Fe} / \mathrm{H}]=-0.5$ and $[\alpha / \mathrm{Fe}]=+0.4$ for $[\mathrm{Fe} / \mathrm{H}] \leqslant-1.0$. We only considered templates with $\log g=5.0$.

The model spectra are broadened to the resolution of our observations and then cross-correlated using the XCSAO routine, which is part of the RVSAO package (Kurtz \& Mink 1998). We ignore rotational broadening and turbulent broadening, which are both much lower than the instrumental resolution (the expected projected rotation speeds are $6.775 \pm 0.056 \mathrm{~km} \mathrm{~s}^{-1}$ and $4.445 \pm 0.039 \mathrm{~km} \mathrm{~s}^{-1}$ for the

\footnotetext{
${ }^{14}$ The $[\mathrm{Fe} / \mathrm{H}]_{K, T 12},[\mathrm{Fe} / \mathrm{H}]_{\mathrm{R} 12}$, and $[\mathrm{M} / \mathrm{H}]_{\mathrm{R} 12}$ indices are determined from the same spectral features and are thus not independent measurements. We adopt the $[\mathrm{Fe} / \mathrm{H}]_{K, T 12}$ index to avoid mixing $[\mathrm{M} / \mathrm{H}]$ and $[\mathrm{Fe} / \mathrm{H}]$, and because $[\mathrm{Fe} / \mathrm{H}]_{K, T 12}$ has a lower uncertainty than $[\mathrm{Fe} / \mathrm{H}]_{\mathrm{R} 12}$ for most of our stars.
}

primary and secondary stars, respectively). The correlation is performed separately for the $(Z+Y), J, H$, and $K$ bands.

We first determine effective temperatures for each of the stars as follows. The normalized cross-correlation peak height $C$ is recorded for each model in the grid, and we fit a paraboloid to values near the peak that is a function of $[\mathrm{Fe} / \mathrm{H}]$ and $T_{\text {eff. }}$ The $T_{\text {eff }}$ value at the peak location is recorded for each band. We then determine best estimates of the $T_{\text {eff }}$, and uncertainties, for each star, still analyzing each bandpass separately, using a similar technique to what was done for the spectroscopic indices. We conducted an MCMC analysis to explore a likelihood function as in Equation (18), with $X$ now being the effective temperature, and replacing $\sigma_{j i}$ with $\alpha_{2} / \mathrm{SN}_{j i}$. Here $\alpha_{2}$ is an additional free parameter and $\mathrm{SN}_{j i}$ is the median $\mathrm{S} / \mathrm{N}$ for spectrum $i$ of $\operatorname{star} j$. This results in $T_{\text {eff }}$ measurements and $1 \sigma$ uncertainties for each star, in each of the four bandpasses. We then combine the four separate bandpasses by using another MCMC and a similar likelihood function. In this case the index $i$ enumerates the different bands, and we use $\sigma_{j i}$ as the $1 \sigma$ uncertainty for band $i$ of star $j$, rather than $\alpha_{2} / \mathrm{SN}_{j i}$. We do this rather than simply taking the weighted average of the four bandpasses, as we found that the scatter between bands exceeded the measurement uncertainties, and this is a straightforward method to account for the additional systematic error. The resulting effective temperatures have uncertainties of $\sim 100 \mathrm{~K}$.

Figure 21 compares the effective temperatures estimated in this manner to the spectral types estimated using the $\mathrm{H}_{2} \mathrm{O}-\mathrm{K}$ indices. In this plot we also show the relations from Bessell (1991), Luhman et al. (2003), and Rajpurohit et al. (2013). We find that our effective temperatures and spectral types are in good agreement with the Rajpurohit et al. (2013) relation, in which effective temperatures are determined by cross-correlating optical spectra against BT-Settl synthetic templates.

Having determined the effective temperature for each star, we next determine the $[\mathrm{Fe} / \mathrm{H}]$ metallicity. We do this in a similar manner to the effective temperatures, except we fix the temperature to the best-estimated value for each star when finding an $[\mathrm{Fe} / \mathrm{H}]$ value that maximizes the correlation for a given spectrum and band. We exclude the $(Z+Y)$ band, which we found to yield $[\mathrm{Fe} / \mathrm{H}]$ values that are systematically lower than the other three bands by $\sim 0.4$ dex. This band also generally has a lower crosscorrelation peak height than the other bands, indicating systematic differences between the models and observations in this wavelength range. The resulting $[\mathrm{Fe} / \mathrm{H}]$ values have uncertainties of $\sim 0.15$ dex. ${ }^{15}$ Figure 22 compares the $[\mathrm{Fe} / \mathrm{H}]$ values so determined to the values based on the Terrien et al. (2012b) $\mathrm{H}$ and $K$-band indices. The two methods yield metallicities that, aside from one significant outlier in NLTT 15867, are fairly consistent. If we remove NLTT 15867, then the cross-correlation-based metallicities are $0.09 \pm 0.03$ dex lower than the T12 metallicities. A difference on this order is not surprising given the different assumed solar abundance patterns on which each metallicity system is based. Comparing to metallicities based on the RojasAyala et al. (2012) indices yields similar results.

For HAT-TR-318-007A and HAT-TR-318-007B we find effective temperatures of $T_{\text {eff,A }}=3190 \pm 110 \mathrm{~K}$ and $T_{\text {eff,B }}=$ $3100 \pm 110 \mathrm{~K}$, respectively, from cross-correlation, and metallicities of $[\mathrm{Fe} / \mathrm{H}]_{\mathrm{A}}=+0.25 \pm 0.13$ and $[\mathrm{Fe} / \mathrm{H}]_{\mathrm{B}}=$ $+0.09 \pm 0.15$, respectively. Combining the metallicities of the primary and secondary components yields a metallicity

\footnotetext{
15 If we do not fix the temperature in finding the $[\mathrm{Fe} / \mathrm{H}]$ values, the results have much larger scatter $(\sim 0.5$ to $1 \mathrm{dex})$ and do not correlate with the metallicities based on the spectroscopic indices.
} 


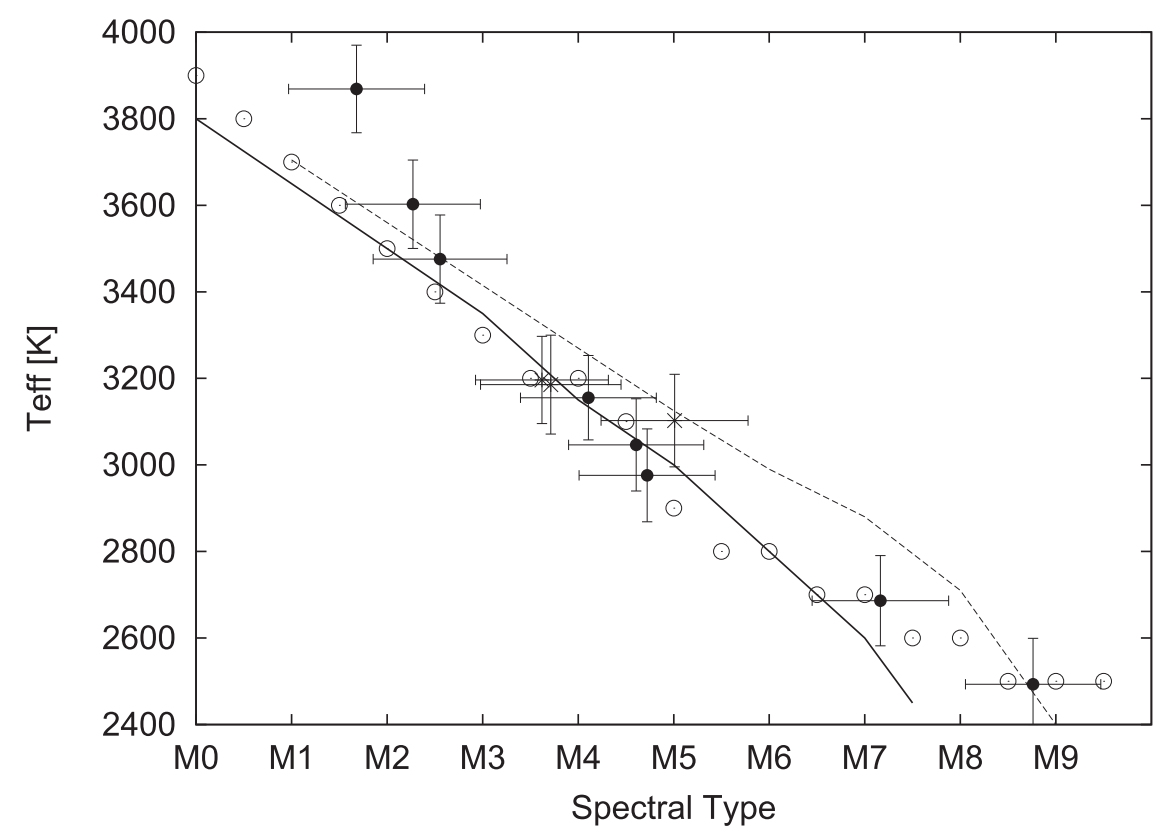

Figure 21. Comparison between the effective temperature determined by cross-correlation against BT-Settl synthetic templates and the spectral type estimated from the $\mathrm{H}_{2} \mathrm{O}-\mathrm{K}$ index and the relation from Rojas-Ayala et al. (2012). The filled circles show standard stars, crosses show the disentangled primary- and secondary-star spectra of HAT-TR-318-007 together with the total eclipse spectrum of HAT-TR-318-007, the solid line shows the relation from Bessell (1991), the dashed line shows the relation from Luhman et al. (2003), and the open circles show the relation from Rajpurohit et al. (2013). Note that the Luhman et al. (2003) relation was calibrated for young stars and is likely not applicable for HAT-TR-318-007.

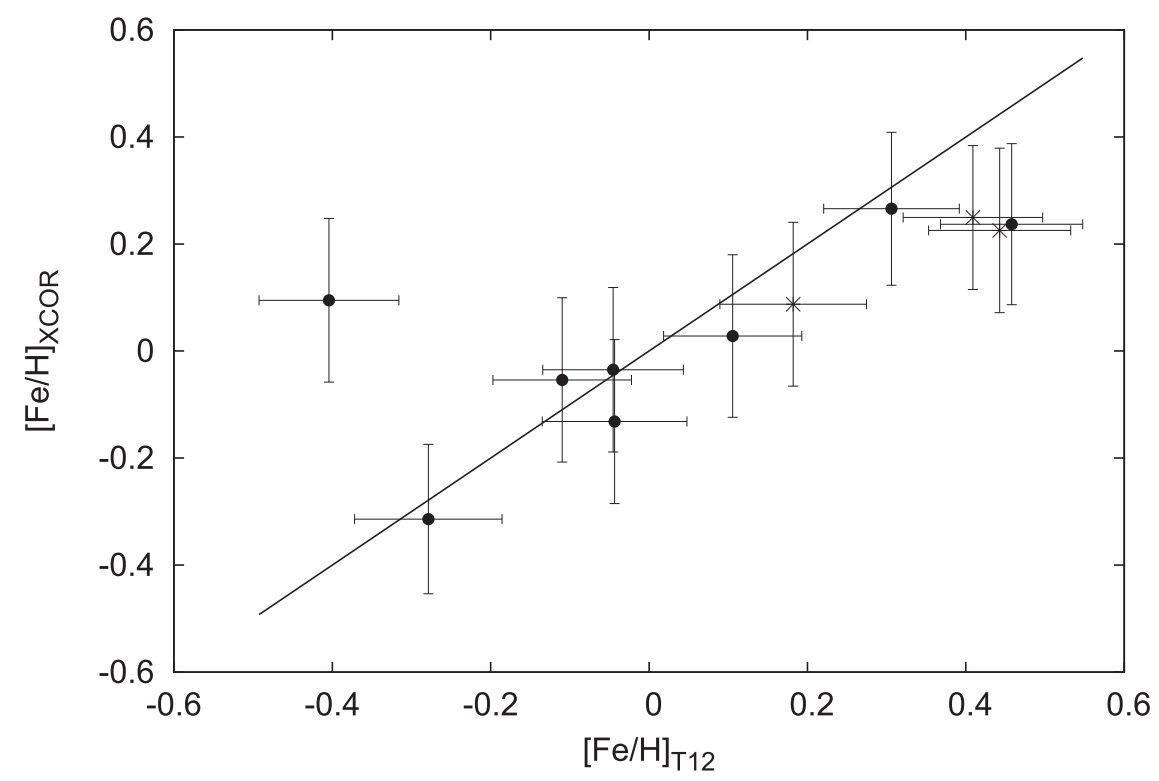

Figure 22. Comparison between the $[\mathrm{Fe} / \mathrm{H}]$ metallicity determined by cross-correlation against BT-Settl synthetic templates (labeled $\left.[\mathrm{Fe} / \mathrm{H}]_{\mathrm{XCOR}}\right)$, and the metallicity estimates from the combined Terrien et al. (2012b) $H$ - and $K$-band indicators (labeled $\left.[\mathrm{Fe} / \mathrm{H}]_{\mathrm{T} 12}\right)$. We exclude the $(Z+Y)$ band from the correlation, which yields systematically lower metallicities than the $J, H$, and $K$ bands. The filled circles show standard stars, and crosses show the disentangled primary- and secondary-star spectra of HAT-TR-318-007 together with the total eclipse spectrum of HAT-TR-318-007. The solid line shows the relation $[\mathrm{Fe} / \mathrm{H}]_{\mathrm{XCOR}}=[\mathrm{Fe} / \mathrm{H}]_{\mathrm{T} 12}$. Excluding NLTT 15867, which is the outlier with $[\mathrm{Fe} / \mathrm{H}]_{\mathrm{T} 12}<-0.4,[\mathrm{Fe} / \mathrm{H}]_{\mathrm{XCOR}}$ is tightly correlated with $[\mathrm{Fe} / \mathrm{H}]_{\mathrm{T} 12}$, but systematically lower by $0.09 \pm 0.03$ dex.

for the system of $[\mathrm{Fe} / \mathrm{H}]=+0.18 \pm 0.10$, which is consistent with the system metallicity determined from the T12 indicators.

\section{ORCID iDs}

J. D. Hartman 나 https://orcid.org/0000-0001-8732-6166

S. N. Quinn (1) https://orcid.org/0000-0002-8964-8377
G. Torres (i) https://orcid.org/0000-0002-5286-0251

D. W. Latham (ib https://orcid.org/0000-0001-9911-7388

A. Shporer (1) https://orcid.org/0000-0002-1836-3120

B. J. Fulton (1) https://orcid.org/0000-0003-3504-5316

M. E. Everett (1i) https://orcid.org/0000-0002-0885-7215

K. Penev (i) https://orcid.org/0000-0003-4464-1371

W. Bhatti $\odot$ https://orcid.org/0000-0002-0628-0088 


\section{References}

Abazajian, K. N., Adelman-McCarthy, J. K., Agüeros, M. A., et al. 2009, ApJS, 182, 543

Ahn, C. P., Alexandroff, R., Allende Prieto, C., et al. 2012, ApJS, 203, 21

Allard, F., Homeier, D., \& Freytag, B. 2011, in ASP Conf. Ser. 448, 16th Cambridge Workshop on Cool Stars, Stellar Systems, and the Sun, ed. C. Johns-Krull, M. K. Browning, \& A. A. West (San Francisco, CA: ASP), 91

Asplund, M., Grevesse, N., Sauval, A. J., \& Scott, P. 2009, ARA\&A, 47, 481

Bakos, G., Noyes, R. W., Kovács, G., et al. 2004, PASP, 116, 266

Bakos, G. Á, Torres, G., Pál, A., et al. 2010, ApJ, 710, 1724

Balaji, B., Croll, B., Levine, A. M., \& Rappaport, S. 2015, MNRAS, 448, 429

Barros, S. C. C., Demangeon, O., \& Deleuil, M. 2016, A\&A, 594, A100

Beatty, T. G., Fernández, J. M., Latham, D. W., et al. 2007, ApJ, 663, 573

Bessell, M. S. 1991, AJ, 101, 662

Bianchi, L., Herald, J., Efremova, B., et al. 2011, Ap\&SS, 335, 161

Birkby, J., Nefs, B., Hodgkin, S., et al. 2012, MNRAS, 426, 1507

Bond, N. A., Ivezić, Ž, Sesar, B., et al. 2010, ApJ, 716, 1

Brown, T. M., Latham, D. W., Everett, M. E., \& Esquerdo, G. A. 2011, AJ, 142,112

Buchhave, L. A., Bakos, G. Á, Hartman, J. D., et al. 2010, ApJ, 720, 1118

Burrows, A., Heng, K., \& Nampaisarn, T. 2011, ApJ, 736, 47

Carter, J. A., Fabrycky, D. C., Ragozzine, D., et al. 2011, Sci, 331, 562

Chabrier, G., Gallardo, J., \& Baraffe, I. 2007, A\&A, 472, L17

Chubak, C., Marcy, G., Fischer, D. A., et al. 2012, arXiv:1207.6212

Claret, A. 2004, A\&A, 428, 1001

Delfosse, X., Forveille, T., Ségransan, D., et al. 2000, A\&A, 364, 217

Dittmann, J. A., Irwin, J. M., Charbonneau, D., et al. 2017, ApJ, 836, 124

Dittmann, J. A., Irwin, J. M., Charbonneau, D., \& Newton, E. R. 2016, ApJ, 818,153

Dotter, A., Chaboyer, B., Jevremović, D., et al. 2008, ApJS, 178, 89

Doyle, L. R., Carter, J. A., Fabrycky, D. C., et al. 2011, Sci, 333, 1602

Eastman, J., Gaudi, B. S., \& Agol, E. 2013, PASP, 125, 83

Edelson, R. A., \& Krolik, J. H. 1988, ApJ, 333, 646

Etzel, P. B. 1981, in Photometric and Spectroscopic Binary Systems, ed. E. B. Carling \& Z. Kopal (Dordrecht: Reidel), 111

Fan, X., Burstein, D., Chen, J.-S., et al. 1996, AJ, 112, 628

Feiden, G. A., \& Chaboyer, B. 2013, in EAS Publications Ser. 64, ed. K. Pavlovski, A. Tkachenko, \& G. Torres (Les Ulis: EDP Sciences), 127

Feiden, G. A., \& Chaboyer, B. 2014, A\&A, 571, A70

Feiden, G. A., Chaboyer, B., \& Dotter, A. 2011, ApJL, 740, L25

Fürész, G. 2008, PhD thesis, Univ. of Szeged

Gaia Collaboration, Vallenari, A., Prusti, T., et al. 2016, A\&A, 595, A2

Giles, H. A. C., Collier Cameron, A., \& Haywood, R. D. 2017, MNRAS, 472, 1618

Gillen, E., Hillenbrand, L. A., David, T. J., et al. 2017, ApJ, 849, 11

Gregory, P. C. 2005, ApJ, 631, 1198

Hadrava, P. 1995, A\&AS, 114, 393

Han, E., Muirhead, P. S., Swift, J. J., et al. 2017, AJ, 154, 100

Hartman, J. D., \& Bakos, G. Á 2016, A\&C, 17, 1

Hartman, J. D., Bakos, G. Á, Noyes, R. W., et al. 2011, AJ, 141, 166

Hilditch, R. W. 2001, An Introduction to Close Binary Stars (Cambridge: Cambridge Univ. Press)

Howell, S. B., Sobeck, C., Haas, M., et al. 2014, PASP, 126, 398

Ilijic, S., Hensberge, H., Pavlovski, K., \& Freyhammer, L. M. 2004, in ASP Conf. Ser. 318, Spectroscopically and Spatially Resolving the Components of the Close Binary Stars, ed. R. W. Hilditch, H. Hensberge, \& K. Pavlovski (San Francisco, CA: ASP), 111

Irwin, J., Charbonneau, D., Berta, Z. K., et al. 2009, ApJ, 701, 1436

Irwin, J. M., Quinn, S. N., Berta, Z. K., et al. 2011, ApJ, 742, 123

Johnson, D. R. H., \& Soderblom, D. R. 1987, AJ, 93, 864
Kaplan, D. L. 2010, ApJL, 717, L108

Kaplan, D. L., Marsh, T. R., Walker, A. N., et al. 2014, ApJ, 780, 167

Keppens, R. 1997, A\&A, 318, 275

Kovács, G., Bakos, G., \& Noyes, R. W. 2005, MNRAS, 356, 557

Kovács, G., Zucker, S., \& Mazeh, T. 2002, A\&A, 391, 369

Kraus, A. L., Douglas, S. T., Mann, A. W., et al. 2017, ApJ, 845, 72

Kraus, A. L., Tucker, R. A., Thompson, M. I., Craine, E. R., \& Hillenbrand, L. A. 2011, ApJ, 728, 48

Kurtz, M. J., \& Mink, D. J. 1998, PASP, 110, 934

Lomb, N. R. 1976, Ap\&SS, 39, 447

Lopez-Morales, M., Orosz, J. A., Shaw, J. S., et al. 2006, arXiv:astro-ph/ 0610225

Luger, R., Kruse, E., Foreman-Mackey, D., Agol, E., \& Saunders, N. 2017, arXiv: 1702.05488

Luhman, K. L., Stauffer, J. R., Muench, A. A., et al. 2003, ApJ, 593, 1093

MacDonald, J., \& Mullan, D. J. 2012, MNRAS, 421, 3084

MacDonald, J., \& Mullan, D. J. 2017, ApJ, 850, 58

Metcalfe, T. S., Mathieu, R. D., Latham, D. W., \& Torres, G. 1996, ApJ, 456, 356

Mohr, P. J., Newell, D. B., \& Taylor, B. N. 2016, RvMP, 88, 035009

Monet, D. G. 1998, BAAS, 30, \#120.03

Morales, J. C., Gallardo, J., Ribas, I., et al. 2010, ApJ, 718, 502

Morales, J. C., Ribas, I., Jordi, C., et al. 2009, ApJ, 691, 1400

Nelson, B., \& Davis, W. D. 1972, ApJ, 174, 617

Planck Collaboration, Ade, P. A. R., Aghanim, N., et al. 2014, A\&A, 571, A16

Popper, D. M., \& Etzel, P. B. 1981, AJ, 86, 102

Prša, A., Harmanec, P., Torres, G., et al. 2016, AJ, 152, 41

Rajpurohit, A. S., Reylé, C., Allard, F., et al. 2013, A\&A, 556, A15

Ramón Iglesias-Marzoa, R., López-Morales, M., Arévalo, M. J., Coughlin, J. L., \& Lázaro, C. 2017, arXiv:1701.06835

Reinhold, T., Reiners, A., \& Basri, G. 2013, A\&A, 560, A4

Ribas, I. 2003, A\&A, 398, 239

Rojas-Ayala, B., Covey, K. R., Muirhead, P. S., \& Lloyd, J. P. 2010, ApJL, 720, L113

Rojas-Ayala, B., Covey, K. R., Muirhead, P. S., \& Lloyd, J. P. 2012, ApJ, 748, 93

Sandquist, E. L., Mathieu, R. D., Brogaard, K., et al. 2013, ApJ, 762, 58

Scargle, J. D. 1982, ApJ, 263, 835

Scharlemann, E. T. 1982, ApJ, 253, 298

Shporer, A. 2017, PASP, 129, 072001

Simcoe, R. A., Burgasser, A. J., Schechter, P. L., et al. 2013, PASP, 125, 270

Simon, K. P., \& Sturm, E. 1994, A\&A, 281, 286

Skrutskie, M. F., Cutri, R. M., Stiening, R., et al. 2006, AJ, 131, 1163

Southworth, J., Maxted, P. F. L., \& Smalley, B. 2004a, MNRAS, 351, 1277

Southworth, J., Zucker, S., Maxted, P. F. L., \& Smalley, B. 2004b, MNRAS, 355,986

ter Braak, C. J. F. 2006, Statistics and Computing, 16, 239

Terrien, R. C., Fleming, S. W., Mahadevan, S., et al. 2012a, ApJL, 760 L9

Terrien, R. C., Mahadevan, S., Bender, C. F., et al. 2012b, ApJL, 747, L38

Torres, G. 2013, AN, 334, 4

Torres, G., \& Ribas, I. 2002, ApJ, 567, 1140

Vanderburg, A., \& Johnson, J. A. 2014, PASP, 126, 948

Windmiller, G., Orosz, J. A., \& Etzel, P. B. 2010, ApJ, 712, 1003

Wright, E. L., Eisenhardt, P. R. M., Mainzer, A. K., et al. 2010, AJ, 140, 1868

Zacharias, N., Monet, D. G., Levine, S. E., et al. 2004, BAAS, 36, 1418

Zechmeister, M., \& Kürster, M. 2009, A\&A, 496, 577

Zhou, G., Bayliss, D., Hartman, J. D., et al. 2014, MNRAS, 437, 2831

Zhou, G., Bayliss, D., Hartman, J. D., et al. 2015, MNRAS, 451, 2263

Zucker, S., \& Mazeh, T. 1994, ApJ, 420, 806 